\title{
5 Koordinationsverfahren bei gleichzeitig relevanten Aktivitäten in Abhängigkeit von der strukturellen (In)Kompatibilität der Beteiligungsweisen
}

In (monoaktiven) Mehrpersonen-Interaktionen stehen Teilnehmende vor der steten Aufgabe, die Beteiligungsweisen ihrer Ko-Interagierenden zu monitoren, um zeitnah und strukturell adäquat darauf reagieren zu können (Goodwin 2018: 151). Gleichzeitig zeigen Interagierende innerhalb einer sozialen Situation einander an, welchen Teilnehmendenstatus sie einander zuschreiben. Eine solche Orientierung eines ${ }^{\star} r$ Teilnehmenden auf eine ${ }^{\star} n$ anderen ko-präsenten Teilnehmenden nennt Goodwin (1981) engagement display. Interagierende mit aktivem engagement display machen sich so für andere Beteiligte kommunikativ verfügbar, während ein disengagement display Nichtverfügbarkeit anzeigt (Goodwin 1981:96). Goodwin beobachtet, dass solche disengagement displays eng damit verbunden sind, inwiefern Teilnehmende einer Einzelaktivität nachgehen. Interagierende müssen also kommunikative Arbeit leisten, wenn sie füreinander engagement displays in solchen Situationen anzeigen wollen, in denen multiple Aktivitäten gleichzeitig relevant sind und sie simultan Einzelaktivitäten realisieren, die potentiell $\mathrm{zu}$ disengagement displays führen können. Um die somit entstehenden Anforderungen mehrerer gleichzeitig relevanter Aktivitäten bearbeiten zu können, bedarf es kommunikativer Verfahren der Teilnehmenden zum Umgang mit diesem interaktionalen Problem. In diesem Kapitel wird daher der Frage nachgegangen, mit welchen Verfahren Teilnehmende die interaktionale Anforderung eines aktiven engagement displays in Situationen mit gleichzeitiger Relevanz multipler Aktivitäten in Abhängigkeit von der strukturellen (In)Kompatibilität der verwendeten Beteiligungsweisen bearbeiten. Da die multiactivity-Forschung, wie im Forschungsstand (Kap. 2.1) dargestellt, bislang vorwiegend die Priorisierung von sprachintensiven Ensembleaktivitäten zugunsten von sprachfreien Einzelaktivitäten fokussiert hat, soll an dieser Stelle untersucht werden, welche strukturellen Bedingungen dazu führen können, dass Interagierende Aktivitäten für andere Aktivitäten aussetzen. Anhand einer systematisch in Bezug auf Einzel- und Ensembleaktivitäten variierten Fallkollektion von 17 Transkripten werden im Zuge dieses Kapitels drei Koordinationsverfahren zur Bearbeitung gleichzeitiger Relevanz von Ensemble- und Einzelaktivitäten untersucht: serielle, quasi-simultane und simultane Koordinierung. Betrachtet werden dazu solche Fälle, in denen ein ${ }^{\star} e$ Teilnehmende`r mindestens zwei Aktivitäten zur selben Zeit koordinieren und für andere Teilnehmende ein engagement display produzieren muss. Dabei steht den Teilnehmenden ein breites Spektrum an Praktiken 
zur Verfügung, um auf dieses interaktionale Problem reagieren zu können (Tab. 1). Das Abbrechen einer Aktivität zugunsten einer anderen stellt dabei die erste Praktik dar. Das dadurch entstehende Nacheinanderbearbeiten der multiplen Aktivitäten (Koordinationsverfahren der Serialität) vollzieht sich im Zuge dessen als geordneter Prozess (5.1). Im Anschluss (5.2) daran folgen Analysen zu Praktiken, mit denen Teilnehmende einzelne Aktivitäten pausiert halten, während sie die Relevanz einer anderen Aktivität bearbeiten (Koordinationsverfahren der Quasi-Simultanität). Abschließend folgen Analysen solcher Fälle, in denen Teilnehmende multiple Aktivitäten gleichzeitig vollziehen (Koordinationsverfahren der Simultanität) (5.3).

Tab. 1: Verfahren der Koordination multipler Aktivitäten.

\begin{tabular}{lll}
\hline Bearbeitung gleichzeitiger Relevanzen in multiplen Aktivitäten \\
\hline Serialität & Quasi-Simultanität & Simultanität \\
\hline $\begin{array}{l}\text { Praktiken der seriellen } \\
\text { Koordination }\end{array}$ & $\begin{array}{l}\text { Praktiken der quasi- } \\
\text { simultanen Koordination }\end{array}$ & $\begin{array}{l}\text { Praktiken der simultanen } \\
\text { Koordination }\end{array}$ \\
\hline 5.1 & 5.2 & 5.3 \\
\hline
\end{tabular}

Um die Koordinationspraktiken der multiplen Aktivitäten miteinander in Relation setzen zu können, beschränkt sich das Setting innerhalb des natürlichen Laboratoriums „Theater“ für die Fallkollektion dieses Kapitels auf die „Vor-Probenphase“. In dieser Phase, in der die Theaterschaffenden auf der Probebühne ankommen und sich für die Probenarbeit des Tages vorbereiten, sind häufig die gleichen Aktivitäten derselben Teilnehmenden zu beobachten. Beteiligte realisieren dabei sowohl sprachfreie Einzelaktivitäten (u.a. SMARTPHONE NUTZEN, TASCHE AUSPACKEN oder KLEIDUNG ABLEGEN) und sprachfreie Ensembleaktivitäten (AUSHÄNDIGEN DER AKTUELLEN STÜCKFASSUNG), als auch sprachintensive Ensembleaktivitäten (wie BEGRÜßEN, BERICHTEN oder VorSCHLAGEN). Die analysierten Transkripte der Fallkollektion sind systematisch so geordnet, dass jeweils strukturell ähnliche Aktivitäten miteinander in Relation gesetzt werden. Das bedeutet hier, dass die Teilnehmenden pro Fall eine sprachintensive Ensembleaktivität mit einer sprachfreien Einzelaktivität (mit jeweils primär haptischen Beteiligungsweisen) koordinieren. Wie sich zeigen wird, besteht ein Zusammenhang zwischen der strukturellen Kompatibilität der Beteiligungsweisen der ko-relevanten Aktivitäten und den von den Teilnehmenden gewählten Koordinierungspraktiken: Je strukturell inkompatibler die Beteiligungsweisen der gleichzeitig relevanten Aktivitäten sind, desto eher brechen Interagierende eine der ko-relevanten Aktivitäten ab. Im Gegensatz ermöglichen strukturell kompatible Beteiligungsweisen die simultane Koordinierung der multiplen Aktivitäten. Dies zeigt sich auch anhand der dieses Kapitel abschließenden Transkripte 16 (Simul- 
tanität zweier sprachfreier Einzelaktivitäten) und 17 (Simultanität einer sprachfreien und einer sprachintensiven Ensembleaktivität). Die dort präsentierten Fälle dienen als Einführung in die Verfahren, die in den folgenden Kapiteln 6 \& 7 eingehend besprochen werden.

\subsection{Praktiken der seriellen Koordination: Abbrechen und Wiederaufnehmen}

Wenn in Interaktionen die Situation eintritt, dass gleichzeitig eine Ensemble- und eine Einzelaktivität relevant werden, haben Teilnehmende zwei Möglichkeiten. Entweder können Interagierende beide Aktivitäten gleichzeitig bearbeiten oder sie können die Aktivitäten als Aktivitätsserie („consecutive ordering of multiple courses of action“, Keisanen, Rauniomaa \& Haddington 2014: 109) aneinanderreihen. Solche seriell koordinierten multiplen Aktivitäten zeichnen sich dadurch aus, dass Teilnehmende zunächst eine der beiden gleichzeitig relevanten Aktivitäten bearbeiten und die andere für die Dauer der Bearbeitung zurückstellen. Im Unterschied zur quasi-simultanen (5.2) bzw. simultanen Koordination (5.3) wird dabei die situative Relevanz einer der Aktivitäten nicht pausiert, sondern aufgehoben und die Aktivität dadurch abgebrochen. Solche Fälle werden zunächst im Rahmen einer Fallkollektion zu Aktivitätsabbrüchen beim Aufrechterhalten anderer Aktivitäten (5.1.1) anhand von drei Transkripten besprochen. Dazu wird zuerst in Transkript 1 der Abbruch einer Einzelaktivität zugunsten einer Ensembleaktivität präsentiert, was der aktuellen Herangehensweise der multiactivity-Forschung entspricht, bevor in Transkript 2 der umgekehrte Fall analysiert wird. Wie sich zeigen wird, ist es für die interaktionale Organisation von Bedeutung, ob eine Einzel- oder eine Ensembleaktivität abgebrochen wird, da Abbrüche intersubjektiv jeweils anders organisiert werden und deren interaktionalen Implikationen sich für Einzel- und Ensembleaktivität unterschieden (Transkript 3). Abgebrochene Aktivitäten können wiederaufgenommen (5.1.2) werden. Auch in dieser Kollektion zweier vergleichbarer Fälle werden die Unterschiede zwischen Einzel- und Ensembleaktivitäten deutlich, die sich einerseits in der sequentiellen Position der Wiederaufnahmen zeigen (Transkript 4) und andererseits von den Teilnehmenden als Wiederaufnahmen gerahmt werden (Transkript 5). Anhand von Wiederaufnahmen wird in Bezug auf die Bearbeitung gleichzeitiger Relevanz von Aktivitäten deutlich, dass Teilnehmende eine Aktivität trotz situativem Abbruch zugunsten einer anderen Aktivität im Hintergrund (Langacker 1987, 2008) weiterhin als relevant behandeln können. Eine Aktivität wird von den Beteiligten in Bezug auf die Fokussierung in den Hintergrund gerückt, wenn sie in Relation zu einer anderen Aktivität weniger stark ausgewählt wird: ,selected 
content is foregrounded relative to unselected content” (Langacker 2008: 68). Wie die Analysen zeigen werden, wählen Beteiligte Aktivitäten dadurch aus, dass sie diese mit spezifischen Beteiligungsweisen bearbeiten. Je nach Art und Anzahl der dabei eingesetzten Beteiligungsweisen, werden Aktivitäten auf diese Weise mehr in den Hintergrund oder in den Vordergrund verschoben, wodurch sie von den Interagierenden als situativ relevanter oder irrelevanter gerahmt werden. Bei Abbrüchen wird eine Aktivität nicht ausgewählt (sie wird in den Hintergrund gerückt) und dadurch (bis zu einer eventuellen Wiederaufnahme) nicht weiterbearbeitet. Auch bei Pausierungen wird die betreffende Aktivität in den Hintergrund gerückt. Allerdings vollzieht sich dies weniger stark als bei Abbrüchen, weil bei Pausierungen mindestens ein Aspekt der Aktivität weiterhin aufrechterhalten bleibt (vgl. 5.2). Bei simultaner Koordinierung (siehe 5.3) werden hingegen alle ko-relevanten Aktivitäten gleichzeitig bearbeitet, weshalb bei diesen Fällen die multiplen Aktivitäten im Vordergrund fokussiert werden. Zunächst soll es um solche Fälle gehen, bei denen Teilnehmende die gleichzeitige Relevanz multipler Aktivitäten bearbeiten, indem sie eine der Aktivitäten durch Abbrüche in den Hintergrund verschieben. Der Grund ist in nahezu allen Transkripten die strukturelle Inkompatibilität der verwendeten Beteiligungsweisen.

\subsubsection{Aktivitätsabbrüche beim Aufrechterhalten anderer Aktivitäten}

Sequenzen, in denen Beteiligte das Ende interaktionaler Einheiten aushandeln, sind regelmäßige Bestandteile sozialer Interaktion. Forschungen zu diesem Phänomen haben unter anderem Beendigungen in Telefongesprächen (Schegloff \& Sacks 1973) oder Verabschiedungen in medizinischen Konsultationen (Heath 1985) in den Blick genommen. Diese Studien zeigen die sequentielle Ordnung bei Aktivitätsabschlüssen durch pre-closings und closing sequences. Diese werden in Face-to-Face-Interaktionen nicht nur verbal, sondern auch multimodal mithilfe von Körperorientierungen (Broth \& Mondada 2013), Objektmanipulationen (Mondada 2015) oder auch Kopf- und Handgesten (Oloff 2018) vollzogen. Besonders in Situationen mit mehreren, gleichzeitig relevanten Aktivitäten sind Abschlüsse bzw. Abbrüche ein interaktional anspruchsvolles Unterfangen, wenn innerhalb des Interaktionsensembles ausgehandelt werden muss, welche der Aktivitäten aufgegeben und welche fortgesetzt werden soll. Aktivitätsabbrüche zugunsten anderer Aktivitäten lassen sich besonders dann beobachten, wenn sich konkurrierende, multiple Aktivitäten strukturell nicht ineinander integrieren lassen (vgl. Keisanen, Rauniomaa \& Haddington 2014). Dies ist unter anderem dann der Fall, wenn multiple Aktivitäten unterschiedliche Aufmerksamkeitsfelder verlangen, wie die gleichzeitige Involvierung in eine Smartphone-Einzelaktivität 
und in eine Face-to-Face-Ensembleaktivität. Eine solche Situation tritt am Morgen des 15. Probentags ein. In dieser steht der Schauspieler (SCM) vor der Herausforderung, sein engagement display bzgl. der NEwSFEED-Einzelaktivität am Smartphone mit dem engagement display der Ensembleaktivität BEGRÜßUNG (Sacks 1972, 1987; Schegloff 2000b, 2007; Heritage 1984) mit dem Dramaturgen (DRA) zu koordinieren. Beide Aktivitäten beinhalten visuelle Beteiligungsweisen, auf die die Teilnehmenden zum Vollzug zurückgreifen. Um die Einzelaktivität IM NEwsFEED LESEN zu realisieren, fokussiert der Schauspieler sein Smartphone und für die Beteiligung an der BEGRÜßUNG stellen die Interagierenden Blickkontakt her. Zusätzlich erfordern beide Aktivitäten haptische Beteiligungsweisen. Dies zeigt sich bei der Einzelaktivität IM NEWSFEED LESEN durch die Objektmanipulation des Smartphones durch den Schauspieler und wird bei der BEGRÜßUNG relevant, als der Dramaturg dem Schauspieler die Hand zum Händedruck reicht. Da beide Aktivitäten somit sowohl visuelle als auch haptische Beteiligungsweisen erfordern und dadurch kein gleichmäßiges engagement display für beide Aktivitäten möglich ist, steht der Schauspieler vor dem Relevanzproblem, welche der Aktivitäten er in den Vordergrund rückt und welche er in den Hintergrund verschiebt.

Tab. 2: Abbruch der Einzelaktivität durch SCM bei Beteiligung an der Ensembleaktivität.

\begin{tabular}{|c|c|c|c|}
\hline Transkript 1 & a) & b) & c) \\
\hline $\begin{array}{l}\text { Einzelaktivität: } \\
\text { NEWSFEED LESEN }\end{array}$ & $\begin{array}{l}\text { engagement display } \\
\text { haptische \& visuelle } \\
\text { Beteiligungsweisen }\end{array}$ & $\begin{array}{l}\text { pausieren } \\
\text { haptische } \\
\text { Beteiligungsweisen }\end{array}$ & $\begin{array}{l}\text { abbrechen } \\
-y^{-}---x\end{array}$ \\
\hline $\begin{array}{l}\text { Ensembleaktivität: } \\
\text { BEGRÜßUNG }\end{array}$ & & $\begin{array}{l}\text { beteiligen } \\
\text { verbale \& visuelle } \\
\text { Beteiligungsweisen }\end{array}$ & \begin{tabular}{l} 
engagement display \\
\hdashline$-y$ verbale, visuelle \\
\& haptische \\
Beteiligungsweisen
\end{tabular} \\
\hline
\end{tabular}

Der Schauspieler löst dieses interaktionale Problem im folgenden Transkriptausschnitt, indem er die Einzelaktivität IM NEWSFEED LESEN zugunsten der konkurrierenden Ensembleaktivität BEGRÜßUNG abbricht. Dieser Abbruch geschieht nicht abrupt, sondern wird von den Teilnehmenden interpersonell koordiniert und semiotisch geordnet vollzogen. Infolgedessen löst der Schauspieler das engagement display der Einzelaktivität auf und macht sich für die Ensembleaktivität verfügbar (Tab. 2). 


\section{Transkript 1: Abbruch der Smartphone- Einzelaktivität IM NeWSFEed LESEN}

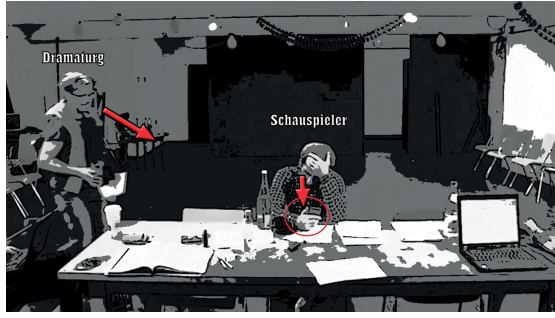

Abb. 1.1: SCM realisiert Smartphone-

Einzelaktivität.

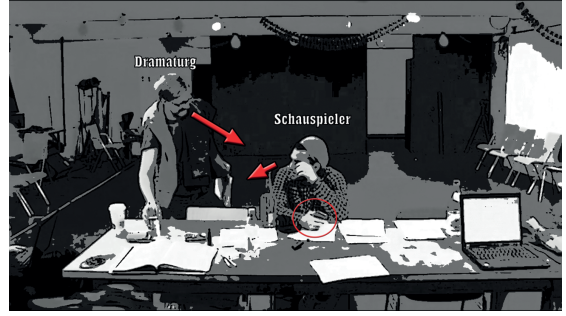

Abb. 1.2: SCM pausiert SmartphoneEinzelaktivität.

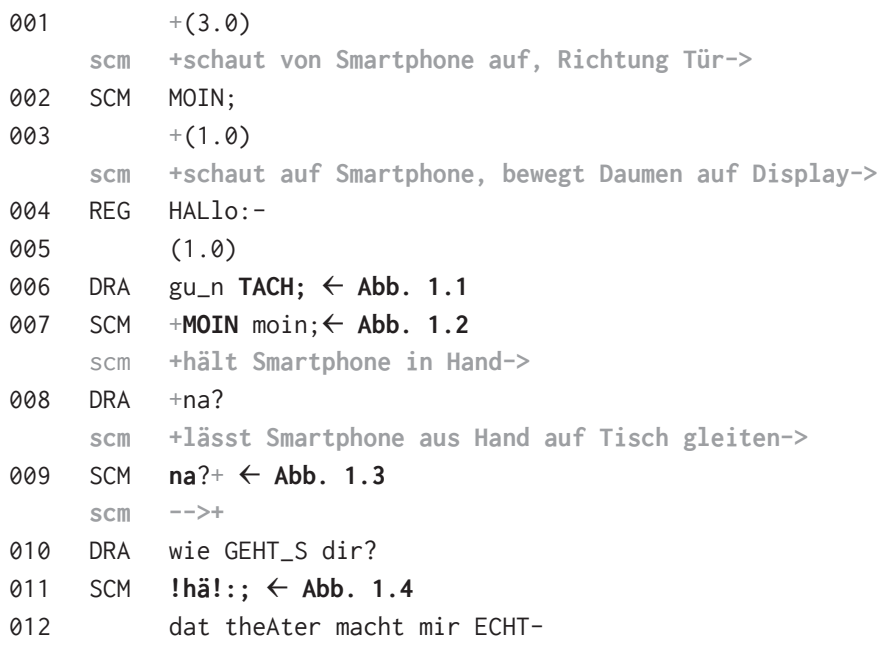

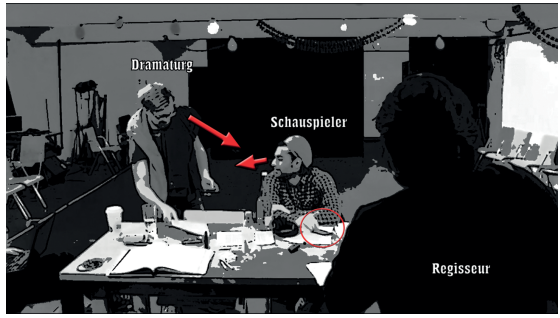

Abb. 1.3: SCM bricht Smartphone-Einzelaktivität ab.

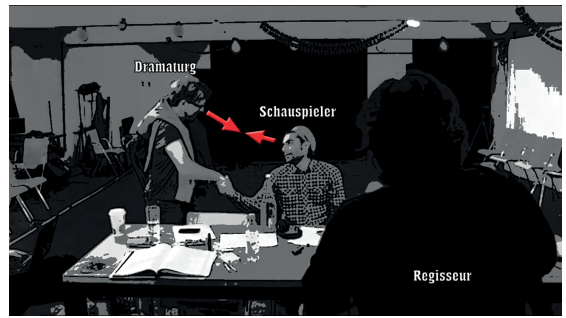

Abb. 1.4: SCM und DRA halten Hände gefasst. 
a) engagement display bzgl. der Einzelaktivität via haptischer und visueller Beteiligungsweisen (Z.001-005): Am Morgen des 15. Probentages sind neben dem Schauspieler lediglich bereits Videograph und Hospitantin anwesend. Alle Teilnehmenden sind seit mehreren Minuten in verbal abstinente Einzelaktivitäten involviert. Der Schauspieler scrollt dabei im Rahmen der haptischen Beteiligungsweise an dieser Aktivität durch einen Newsfeed eines sozialen Netzwerks und liest einzelne Meldungen (visuelle Beteiligungsweise). Als Schritte zu hören sind, blickt er auf und schaut Richtung der Tür (Z.001), durch die der Dramaturg und der Regisseur die Probebühne betreten. Der Schauspieler begrüßt die beiden (Z.002) und wendet sich aber wieder seiner Newsfeed-Einzelaktivität zu, als der Gegengruß ausbleibt (Z.003). Dieses engagement display bzgl. Einzelaktivität hält der Schauspieler auch dann aufrecht, als der Regisseur seinerseits einen Gruß produziert (Z.004).

b) duales engagement display bzgl. beider Aktivitäten durch Aufteilen der Beteiligungsweisen (Z.006-007): Erst als der Dramaturg sich dem Tisch nähert, an dem der Schauspieler Platz genommen hat (Abb. 1.1) und ebenfalls einen Gruß realisiert (Z.006), verändert der Schauspieler sein engagement display. Er erwidert den Gruß des Dramaturgen im Rahmen der verbalen Beteiligungsweise an der BEGRÜßUNG (Z.007). Während er mit seinem Gegengruß die als Paarsequenz organisierte Ensembleaktivität BEGRÜßUNG bearbeitet, löst der Schauspieler seinen Blick von seinem Smartphone, das er weiterhin in der Hand hält, und verfolgt visuell, wie der Dramaturg sein Wasserglas auf den Tisch stellt (Abb. 1.2). Der Schauspieler stoppt dadurch seine visuelle Beteiligungsweise an der Smartphone-Einzelaktivität, zeigt aber weiterhin ihre fortbestehende situative Relevanz an, indem er das Smartphone im Rahmen der haptischen Beteiligungsweise in der Hand hält. Da er die Bindung mit dem Fokusobjekt der Einzelaktivität aufrechterhält, sie aber nicht weiterbearbeitet (er scrollt nicht mehr mit seinem Daumen), pausiert der Schauspieler dadurch seine Einzelaktivität. Er reagiert somit auf die simultane Relevanz zweier Aktivitäten (BEGRÜßUNG vs. IM NEWSFEED LESEN).

c) Abbruch der Einzelaktivität durch Modifikation des engagement displays bzgl. der Ensembleaktivität via verbaler, visueller und haptischer Beteiligungsweisen (Z.008-012): Den pausierten Zustand der Einzelaktivität gibt der Schauspieler auf, als der Dramaturg sowohl eine verbale als auch eine haptische Bearbeitung der Ensembleaktivität relevant setzt. Dies realisiert er, indem er dem Schauspieler die Hand entgegenhält und mit einer Fragepartikel mit stark steigendem prosodischen Verlauf (,na?“, Z.008) eine neue Paarsequenz evoziert. Der Schauspieler löst diese doppelte Relevantsetzung der Ensembleaktivität dadurch ein, dass 
er mit der gleichen Partikel (Z.009) reagiert und in diesem Zuge sein Smartphone auf den Tisch gleiten lässt, wodurch seine rechte Hand für den Händedruck verfügbar wird (Abb. 1.3). Der Schauspieler ergreift die ausgestreckte Hand des Dramaturgen (Abb. 1.4), nimmt mit ihm Blickkontakt auf und bearbeitet dessen Frage „wie GEHT_S dir?“(Z.010) mit einer gedehnten und in der Lautstärke intensivierten Partikel (Z.011), mit der er eine dispräferierte Antwort (Pomerantz 1984) projiziert, die er im Folgenden ausführt ${ }^{28}$ (Z.012).

Die Bearbeitung der Interaktionsaufgabe der BEGRÜßUNG erfordert hier den Abbruch der Einzelaktivität des Schauspielers, die für ihre Aufrechterhaltung zum einen die visuelle Orientierung auf das Smartphonedisplay voraussetzt und zum anderen eine haptische Bindung mit dem Objekt benötigt. Die ko-relevanten Aktivitäten BEGRÜßEN und IM NEWSFEED LESEN verhalten sich hier strukturell inkompatibel zueinander, da beide Aktivitäten im Kern auf dieselben visuellen und haptischen Beteiligungsweisen zurückgreifen. Der Schauspieler leistet den Abbruch in zwei Schritten, indem er zunächst die visuelle und danach die haptische Beteiligung an der Aktivität aufgibt und die freiwerdenden multimodalen Ressourcen auf die Ensembleaktivität überträgt. Dazu hält er das Smartphone im ersten Schritt noch in der Hand. Dies ermöglicht es ihm, die Einzelaktivität problemlos wieder aufnehmen zu können, sobald die BEGRÜßUNG abgeschlossen ist. Die Einzelaktivität kann zu diesem Zeitpunkt als pausiert ${ }^{29}$ gelten. Als der Dramaturg jedoch die BEGRÜßUng um eine Frage erweitert und dem Schauspieler die Hand reicht, womit der Dramaturg die (konditionelle) Relevanz der Ensembleaktivität aktualisiert, priorisiert der Schauspieler diese Ensembleaktivität, indem er im zweiten Schritt das Smartphone aus der Hand gleiten lässt und im Folgenden die Frage monoaktiv bearbeitet. Damit werden in der Hand halten und aus der Hand gleiten lassen in dieser Situation als zwei Praktiken erkennbar, mit denen der Schauspieler den schrittweisen Übergang von engagement display bzgl. der Einzelaktivität zu engegement display bzgl. der Ensembleaktivität herstellt. Das Verschieben des engagement displays von einer Aktivität auf eine andere kann entweder in einer monoaktiven Situation im Rahmen von activity transitions zwischen zwei Ensembleaktivitäten geschehen (Robinson \& Stivers 2001; Reed, Reed \& Haddon 2013; Reed 2015) oder wie im obenstehenden Ausschnitt von einer Einzelaktivität zu einer Ensembleaktivität. Der Grad, in welchem Teilnehmende eine laufende Aktivität für andere Beteiligte intersubjektiv nachvollziehbar gestalten,

28 Es gibt Probleme mit der Theaterleitung bei der bürokratischen Abwicklung seiner Rechnungen. 29 Die Praktik des Pausierens multipler Aktivitäten zur Bearbeitung gleichzeitiger Relevanz wird in 5.2 eingehend diskutiert. 
ist dabei von zentraler Bedeutung für dieses koordinative Verfahren und zeigt sich in der Struktur der Teilnehmendenkonstellation einer Aktivität.

Im umgekehrten Fall des Abbruchs einer Ensembleaktivität zugunsten einer Einzelaktivität verhält es sich anders, wie der folgende Ausschnitt in Transkript 2 zeigt. In diesem zieht der Schauspieler (SCM) seine Beteiligung an der Ensembleaktivität ERKLÄREN zurück, zeigt lediglich engagement display bezüglich seiner Einzelaktivität RUCKSACK AUSPACKEN und lässt damit den Hospitanten (HOM) als alleinigen Teilnehmer dieser Aktivität zurück, bis sich die ko-präsente Schauspielerin (SCW) und der ebenfalls anwesende Videograph (VID) an der Aktivität beteiligen und sie dadurch wieder den Status einer Ensembleaktivität erhält. Bei diesem Abbruch zieht sich der Schauspieler im Gegensatz zum vorherigen Transkript 1 abrupt aus der dyadischen Ensembleaktivität zurück, ohne sie in irgendeiner Weise als pausiert zu markieren oder einen Account für seinen Rückzug aus der Ensembleaktivität zu liefern. Dieses disengagement display hat insofern interaktionale Konsequenzen, als der übrigbleibende Teilnehmer daraufhin ebenfalls Hinweise liefert, sich aus der laufenden Aktivität zurückzuziehen.

Tab. 3: Abbruch der Ensembleaktivität durch SCM bei Beteiligung an der Einzelaktivität.

\begin{tabular}{|c|c|c|c|}
\hline Transkript 2 & a) & b) & c) \\
\hline \multirow{4}{*}{$\begin{array}{l}\text { Einzelaktivität: } \\
\text { AUSPACKEN } \\
\text { (bzw. LESEN) }\end{array}$} & & engagement display & engagement display \\
\hline & & 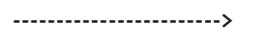 & 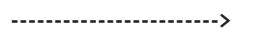 \\
\hline & & haptische & haptische \& visuelle \\
\hline & & Beteiligungsweise & Beteiligungsweisen \\
\hline \multirow{4}{*}{$\begin{array}{l}\text { Ensembleaktivität: } \\
\text { ERKLÄREN }\end{array}$} & engagement display & engagement display & abbrechen \\
\hline & 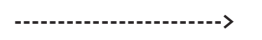 & 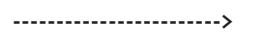 & --.-------x \\
\hline & verbale \& visuelle & visuelle & \\
\hline & Beteiligungsweisen & Beteiligungsweise & \\
\hline
\end{tabular}

Der Abbruch im untenstehenden Transkriptausschnitt folgt demselben Muster, das bereits in Transkript 1 gezeigt wurde: a) singuläres engagement display, b) duales engagement display durch Aufteilen der Beteiligungsweisen und c) singuläres engagement display nach Relevanzverschiebung. Das Beispiel zeigt aber im Vergleich zum ersten Transkript die unterschiedlichen Implikationen, die Abbrüche von Ensemble- zugunsten von Einzelaktivitäten für die laufende Interaktion haben können: Der Abbruch einer Ensembleaktivität ist für die intersubjektive Gestaltung einer Interaktion deutlich riskanter als der Abbruch einer Einzelaktivität, die zunächst nur für die Person unmittelbare Auswirkungen hat, die sich daran beteiligt (Tab. 3). 


\section{Transkript 2: Abbruch der Ensembleaktivität ERKLÄRUNG ohne Account}

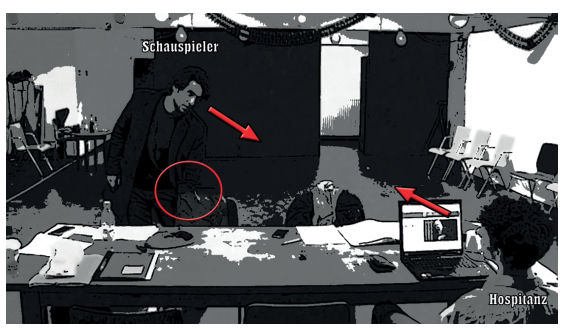

Abb. 2.1: SCM nickt beim ENTPACKEN.

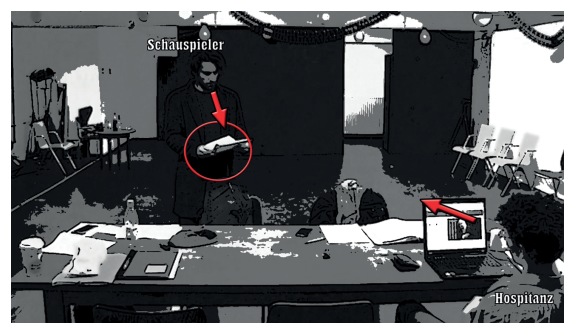

Abb. 2.2: SCM LIEST im Text, HOM ERKLÄRT weiter.

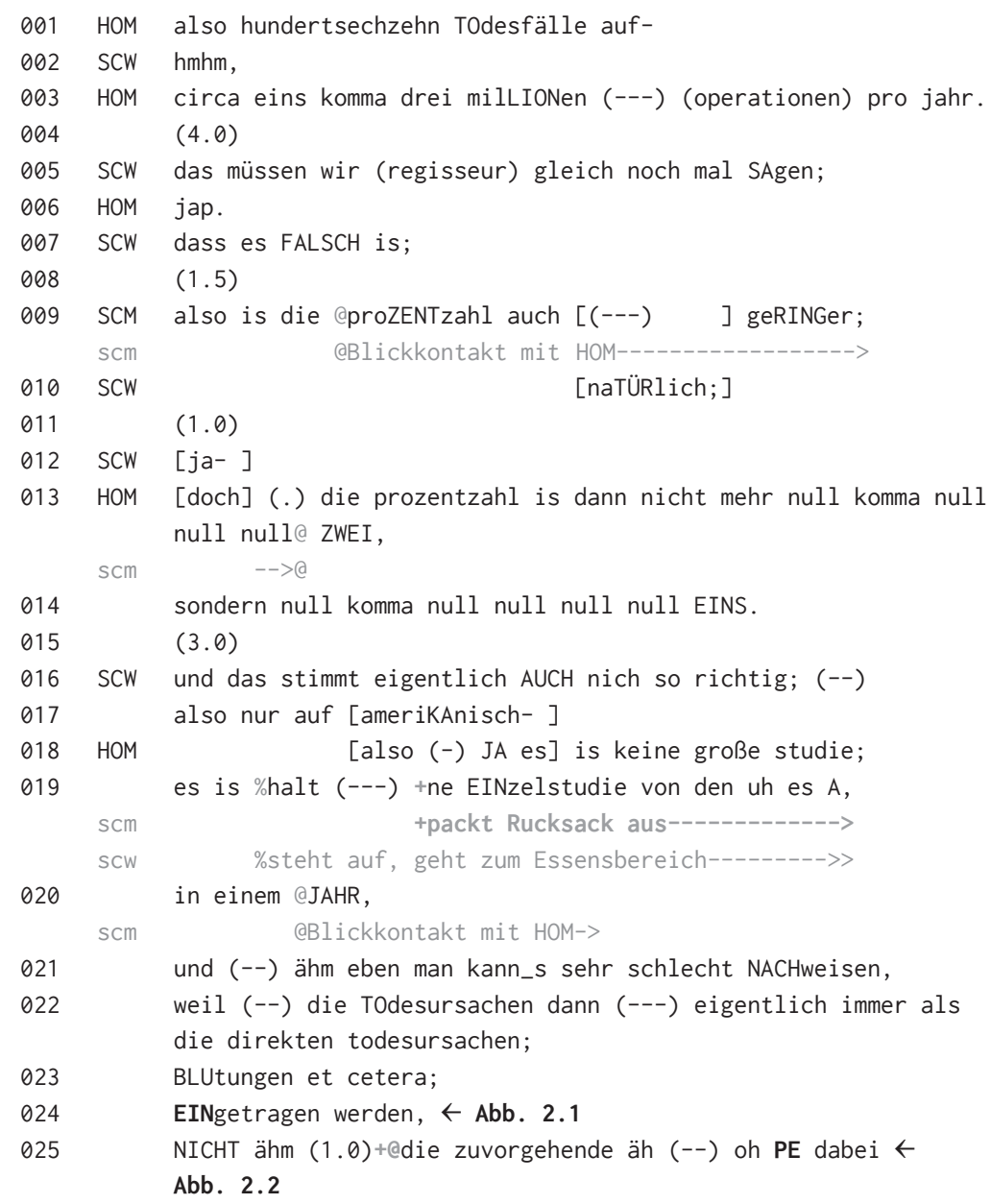




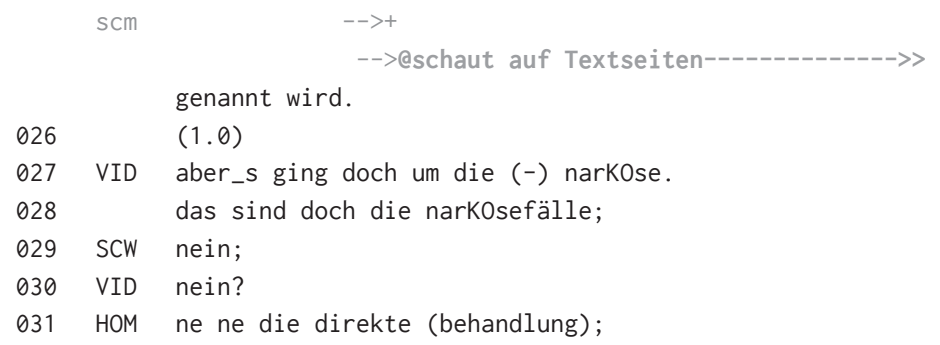

a) engagement display bzgl. der Ensembleaktivität via verbaler \& visueller Beteiligungsweisen (Z.001-018): In der beobachteten Stückentwicklung arbeiten die Beteiligten nicht nur an der Inszenierung auf der Probebühne, sondern recherchieren kontinuierlich Fakten, um damit das Stück in einem realen Gesellschaftsdiskurs verorten zu können. Der Hospitant (HOM) hat es sich zur Aufgabe gemacht, diese Fakten zu überprüfen und eröffnet der Schauspielerin (SCW) in der Vor-Probenphase der 12. Probe eine fehlerhafte Information (eine vom Regisseur recherchierte Prozentzahl ist $\mathrm{zu}$ hoch) gefunden $\mathrm{zu}$ haben (Z.001-008). Als der Hospitant mit seiner ERKLÄRUNG bzgl. des Faktes geendet hat, greift der Schauspieler sie noch einmal auf, während er seinen Rucksack AUSPACKT (Z.009) und dafür vor allem auf haptische Beteiligungsweisen an dieser Einzelaktivität zurückgreift. Im Verlauf der Sequenz zieht sich der Schauspieler aus der Ensembleaktivität ERKLÄREN zurück, während er weiterhin seine Einzelaktivität AUSPACKEN aufrechterhält. Die von der Schauspielerin als Frage behandelte Äußerung des Schauspielers (,also is die proZENTzahl auch geRINGer“, Z.009) ist damit seine erste (und einzige) verbale Beteiligung an der Ensembleaktivität. Der Schauspieler nimmt an dieser Stelle die vorerst abgeschlossene Ensembleaktivität auf, verweist thematisch auf die falsche Information und orientiert sich im Rahmen der visuellen Beteiligungsweise via Blickkontakt sowie body torque (Schegloff 1998) auf den Hospitanten, wodurch ein interaktionales withness display (vgl. Kendon 1990: 250) entsteht. Die Schauspielerin bejaht die Schlussfolgerung des Schauspielers („naTÜRlich“, Z.010) und zeigt damit ihrerseits an, dass sie sich ebenfalls als Teil (with, Goffman 2010 [1971]: 19-21) des Interaktionsensembles versteht. Blickkontakt mit dem fragenden Schauspieler aufrechterhaltend bestätigt der Hospitant daraufhin ebenfalls die Schlussfolgerung des Schauspielers und liefert Informationen zur falschen wie zur korrekten Prozentzahl (Z.013/14). Obwohl der Schauspieler daraufhin den Blickkontakt abbricht und seine AuSPACK-Aktivität vorbereitet (indem er den Rucksack auf den Stuhl stellt), behandeln ihn die Schauspielerin und der Hospitant trotz ausbleibender körperlicher Orientierung oder verbalen Rezipientensignalen im weiteren Verlauf der Sequenz weiterhin als Beteiligten der Ensembleaktivität (Z.015-19). Das zeigt sich 
unter anderem daran, dass beide das eigentlich bereits mit einer abgesprochenen Folgehandlung (den Regisseur über seinen Fehler zu informieren, vgl. Z.005-7) abgeschlossene Thema im Rahmen kollaborativer Expansionen ko-konstruierend erweitern (Günthner 2015: 60-61) und damit Kontextualisierungshinweise bzgl. der withness des Interaktionsensembles liefern (Z.016-18).

b) duales engagement display bzgl. beider Aktivität durch Aufteilen der Beteiligungsweisen (Z.019-021): Auch als der Schauspieler beginnt, seinen Rucksack AUSZUPACKEN und die Schauspielerin vom Tisch aufsteht, um sich beim Essensbereich Kaffee einzugießen, wird er vom Hospitanten weiterhin als Teilnehmer der Ensembleaktivität adressiert (Z.019). Der Schauspieler liefert ein engagement display bzgl. der Ensembleaktivität dadurch, dass er seine Einzelaktivität so organisiert, dass er während der haptischen Beteiligung am AUSPACKEN Blickkontakt (visuelle Beteiligungsweise) mit dem Hospitanten aufnehmen kann (Z.020-21, Abb. 2.1). Damit teilt er seine Beteiligungsweisen so auf die gleichzeitig relevanten Aktivitäten auf, dass er für beide Aktivitäten ein engagement display herstellen kann. Dass dies für das Fortführen der Aktivitäten bedeutsam ist, zeigt sich zum einen darin, dass der Schauspieler während seiner Beteiligung an der Ensembleaktivität weiterhin AUSPACKEN kann und zum anderen in der Reaktion des Hospitanten, der weiterhin im Rahmen der Ensembleaktivität verbale Beiträge produziert, solange der Schauspieler dieses engagement display aufrechterhält. Diese Konfiguration der simultan relevanten Aktivitäten AUSPACKEN und ERKLÄREN hält der Schauspieler aufrecht, bis seine AuSPACK-Einzelaktivität in Bezug auf die Verfügbarkeit multimodaler Ressourcen einen kritischen Status erreicht: Er zieht die lose Blattsammlung der aktuellen Stückfassung heraus und richtet seinen Blick darauf (Abb. 2.2).

c) Abbruch der Ensembleaktivität durch Modifikation des engagement displays bzgl. der Ensembleaktivität via visueller und haptischer Beteiligungsweisen (Z.022-029): Die AusPACK-Einzelaktivität des Schauspielers geht in eine LESE-Einzelaktivität über, die er für die nächsten dreißig Sekunden aufrechterhält. Damit erreicht auch die Fortführung der Ensembleaktivität einen kritischen Zustand, da sie nun bis auf den Hospitanten keine weiteren Teilnehmenden zu haben scheint. Der Hospitant reagiert auf diese Veränderung im zur Bearbeitung der Interaktionsaufgabe erforderlichen engagement display des Schauspielers und damit in der Teilnehmendenrahmung der Aktivität, indem er (den Schauspieler weiterhin anschauend) seinen mit Disfluenzmarkern (Partikel äh und Sprechpause) gestreckten Turn zum Ende führt. Er markiert das Ende seines verbalen Interaktionsbeitrags mit stark fallender Tonhöhenbewegung (Z.025) und einer einsekündigen Phase verbaler Abstinenz, in der er den Schauspieler nach wie vor anschaut, 
aber bezogen auf die Aktivität keine weitere (verbale) Beteiligung leistet (Z.026). Die Ensembleaktivität wird schließlich wieder relevant gesetzt, als der Videograph einen Deutungsversuch bzgl. der vom Hospitanten vorgestellten Statistik produziert (Z.027-28). Die Schauspielerin lehnt diese Interpretation ab (Z.029), worauf alle Anwesenden (bis auf den Schauspieler) eine gemeinsame DiskusSIONs-Ensembleaktivität herstellen, in der sie Interpretationsmöglichkeiten der ERKLÄRTEN Statistik aushandeln.

In dieser Sequenz verschiebt der Schauspieler sein engagement display von der Ensembleaktivität, an der er sich unter anderem verbal und via Blickkontakt beteiligt, zu einer Einzelaktivität, die analog zum Transkript 1 die visuelle Orientierung auf ein Aufmerksamkeitsfeld erfordert. Anders als jedoch in Transkript 1 vermag der Schauspieler hier seine zwei Aktivitäten kurzzeitig so zu organisieren, dass er Blickkontakt mit dem Hospitanten im Rahmen der Ensembleaktivität halten kann, während er gleichzeitig seine Einzelaktivität per haptischer Beteiligungsweise bearbeitet (Abb. 2.1). Die Orientierung auf die Textseiten, die er im Anschluss an das AUSPACKEN LIEST, erlaubt eine solche simultane Konfiguration nicht: Er kann entweder Blickkontakt mit dem Hospitanten halten (und damit ein engagement display bzgl. der Ensembleaktivität leisten) oder LESEN. Darüber hinaus ist im Unterschied zur Sequenz in Transkript 1 in diesem Ausschnitt keine schrittweise Vorbereitung des Aktivitätsabbruchs erkennbar. Vielmehr zieht sich der Schauspieler abrupt aus der Ensembleaktivität zurück, ohne sie in irgendeiner Weise als pausiert zu markieren. Dieser Abbruch hat unmittelbar Auswirkung auf den einzig verbleibenden Teilnehmer der Aktivität, der seinen Interaktionsbeitrag beendet, sobald der Schauspieler keine Beteiligung an der Ensembleaktivität mehr anzeigt. Der Austritt des Schauspielers aus der Ensembleaktivität bleibt gänzlich unmarkiert und lässt den anderen Beteiligten der Ensembleaktivität als last man standing allein und mit einer potentiellen Gesichtsbedrohung (Brown \& Levinson 1987) zurück. Auf das Fehlen der Involvierungsanzeige des Schauspielers reagiert der Hospitant zunächst mit Disfluenzmarkern und dann, indem er die Ensembleaktivität zum nächstmöglichen Zeitpunkt beendet. Das fehlende participation-Display (Goodwin 2000d; Goodwin \& Goodwin 2004; Goodwin 2007) einer Beteiligung an der Ensembleaktivität ist für den Hospitanten eine Ressource, aufgrund derer er sich für das Abschließen der Ensembleaktivität entscheidet. In diesem Fall liegt es an den anderen Anwesenden, die Ensembleaktivität durch eigene Beteiligung aufrechtzuerhalten (was durch Schauspielerin und Videographen schließlich auch geschieht). Die Ensembleaktivität verliert damit innerhalb des Interaktionsensembles an Relevanz, als einer der Teilnehmenden seine Beteiligung abbricht und die Ensembleaktivität dadurch kurzzeitig zu einer Einzelaktivität wird. 
Der Rückzug des Schauspielers aus der Ensembleaktivität durch eine ausbleibende Beteiligung an der Aktivität geschieht in Transkript 2 ohne Account seitens des Schauspielers. Dass ein solcher Account an der Bruchkante einer Aktivität interaktionale Implikationen hat, zeigt das folgende Transkript. In diesem BERICHTET der Regisseur dem Dramaturgen von der letzten Probe, als das Smartphone des Dramaturgen klingelt. Dieser steht auf und hält dem Regisseur das Smartphone entgegen, während er die Probebühne verlässt. Dieses Smartphonezeigen interpretiert der Regisseur als Account für den Aktivitätsabbruch durch den Dramaturgen und orientiert sich im Folgenden auf Schauspielerin und Schauspieler. Die interaktionale Rahmung des disengagement displays bzgl. der laufenden Ensembleaktivität mithilfe eines Accounts ermöglicht den Beteiligten eine dynamische Modifikation der Teilnehmendenrahmung, die sich in diesem Beispiel in der Fortsetzung der Aktivität mit anderen Teilnehmenden ausdrückt (Tab. 4).

Tab. 4: Abbruch der Ensembleaktivität durch DRA bei Beteiligung an der Einzelaktivität.

\begin{tabular}{|c|c|c|c|}
\hline Transkript 3 & a) & b) & c) \\
\hline Einzelaktivität: & & engagement display & engagement display \\
\hline SMARTPHONE- & & - & - \\
\hline \multirow[t]{2}{*}{ ReLEVANZ BEARBEITEN } & & proxemische & gestische \\
\hline & & Beteiligungsweise & Beteiligungsweise \\
\hline Ensembleaktivität: & engagement display & pausieren & abbrechen \\
\hline \multirow[t]{3}{*}{ BERICHTEN } & - & ||--||-11 & - \\
\hline & visuelle & & \\
\hline & Beteiligungsweise & & \\
\hline
\end{tabular}

\section{Transkript 3: Abbruch der Ensembleaktivität BERICHTEN mit Account}

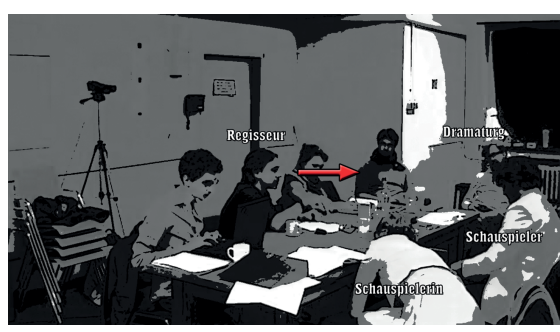

Abb. 3.1: REG adressiert DRA.

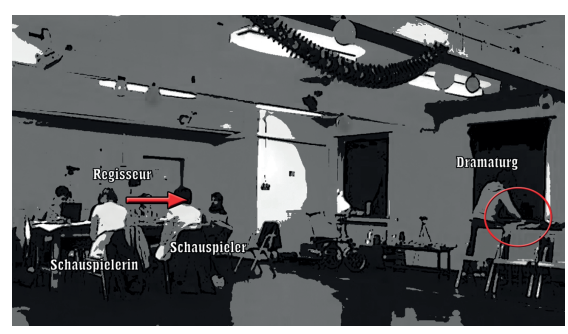

Abb. 3.2: DRA greift sein Smartphone. 


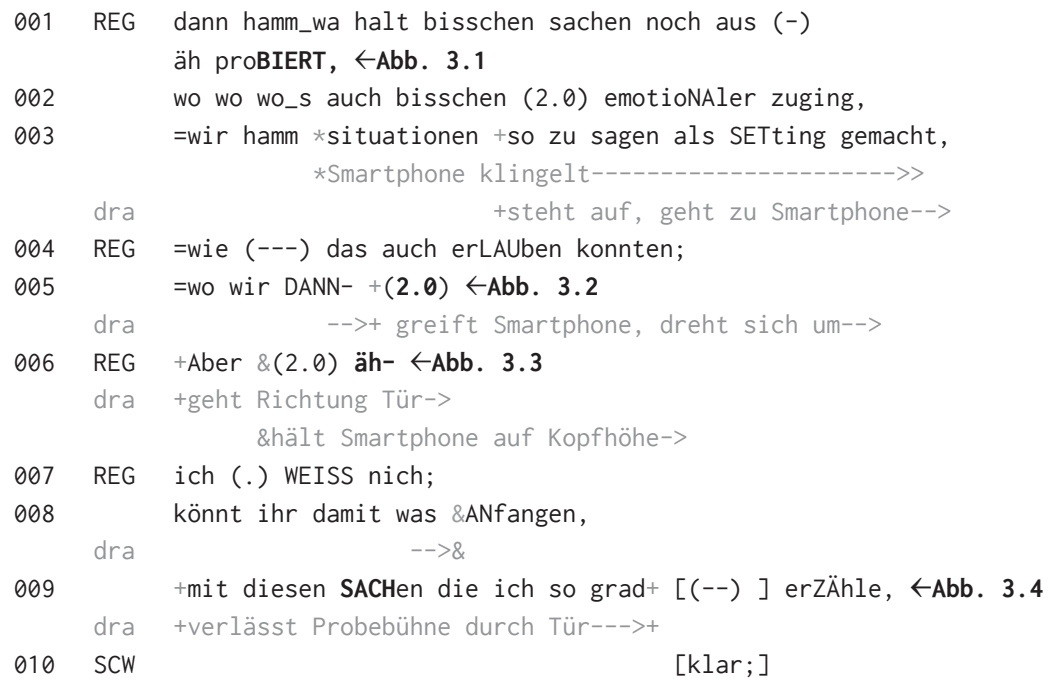

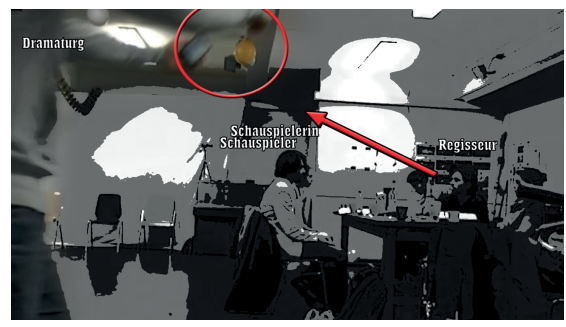

Abb. 3.3: DRA zeigt auf das Smartphone.

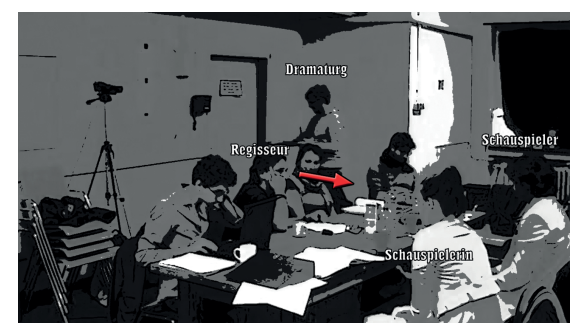

Abb. 3.4: REG adressiert SCW \& SCM.

a) engagement display bzgl. der Ensembleaktivität via visueller Beteiligungsweise (Z.001-003): Der Regisseur richtet seinen BERICHT visuell und sprachlich an den Dramaturgen (Z.001-2), der dem Regisseur (im Unterschied zur Schauspielerin und dem Schauspieler) per Körperorientierung und Blickkontakt ein display of recipiency (Heath 1986) anbietet (Abb. 3.1). Währenddessen klingelt das Smartphone des Dramaturgen, der aufsteht und sich Richtung seines Smartphones bewegt (Z.003). Der Regisseur setzt seinen BERICHT fort (Z.004-5) und folgt dem Gang des Dramaturgen zum Smartphone visuell (Abb. 3.2). Wie beim Hospitanten im vorherigen Transkriptausschnitt macht sich auch hier das fehlende engagement display des Interaktionspartners in Form von Disfluenzmarkern bemerkbar. Neben häufigen Sprechpausen (Z.004, Z.005, Z.006, Z.007) zeigen sich diese in den Äußerungen des Regisseurs auch im Rahmen syntagmatisch inadäquater Äußerungsemergenz (Z.004) sowie als Anakoluthe (Z.005 \& Z.006). 
b) Pausieren der Ensembleaktivität bei engagement display bzgl. der Einzelaktivität via proxemischer Beteiligungsweise (Z.005-006): Der Regisseur reagiert auf das fehlende Involvierungsdisplay des Dramaturgen, indem er die verbale Beteiligung an seinem Bericht für zwei Sekunden pausiert (Z.005). Damit bearbeitet der Regisseur die gleichzeitige Relevanz der beiden Aktivitäten, indem er die Ensembleaktivität pausiert, bis der Status der Einzelaktivität (und damit das engagement display bzgl. der Ensembleaktivität) geklärt ist. Dies wird deutlich daran, dass der Regisseur ansetzt in seinem Bericht fortzufahren („Aber“, Z.006) als der Dramaturg das Smartphone greift und sich damit umdreht. Der Dramaturg bewegt sich jedoch zügig Richtung Tür (und nicht zurück Richtung des Regietisches) und hält dabei das Smartphone mit der linken Hand vor sich, während er mit der rechten Hand auf das Smartphone zeigt (Abb. 3.3). Diese Objekt-KörperKonfiguration monitort der Regisseur für etwa zwei Sekunden, während er seine verbale Beteiligungsweise abermals pausiert (Z.006).

c) Abbruch der Ensembleaktivität durch Relevantsetzen der Einzelaktivität via gestischer Beteiligungsweise als Account (Z.007-010): Der Regisseur behandelt die Geste, mit der der Dramaturg auf das Smartphone zeigt und die SmartphoneEinzelaktivität damit relevant setzt, als Account dafür, dass der Dramaturg seine Beteiligung an der Ensembleaktivität abbricht. Dies zeigt sich in der Reaktion des Regisseurs, der sich im Anschluss an die Geste des Dramaturgen visuell auf die Schauspielerin und den Schauspieler (Abb. 3.4) orientiert, die er direkt pronominell adressiert (,könnt ihr damit was ANfangen“, Z.008). Die beiden Adressierten zeigen darauf nicht nur in ihrer auf- und auf den Regisseur ausgerichteten Positur an, dass sie sich jetzt als ratifizierte Teilnehmende der Ensembleaktivität verstehen (vgl. Abb. 3.1 vs. Abb. 3.3), sondern sie beteiligen sich darüber hinaus auch verbal an der Ensembleaktivität (Z.010). Damit zieht das auf das Smartphone Zeigen des Dramaturgen eine Veränderung im Adressatenzuschnitt (Betz 2015; Deppermann 2014a, 2015; Deppermann \& Blühdorn 2013; Deppermann \& Schmitt 2009; Hitzler 2013; Imo 2015; Schmitt \& Knöbl 2013, 2014) des Regisseurs nach sich. Der Dramaturg liefert unter anderem mithilfe der Zeigegeste einen Account für sein Ausscheiden aus der Ensembleaktivität, auf die der Regisseur reagiert, indem er den Adressatenzuschnitt der Aktivität modifiziert. Damit wechselt der Adressatenzuschnitt innerhalb des Regieturns vom Dramaturgen auf Schauspielerin und Schauspieler an genau jener sequentiellen Position, an der der Dramaturg den sprachfreien Account für seinen Rückzug aus der Ensembleaktivität liefert, indem er seine Einzelaktivität relevant setzt.

Vergleicht man den accountlosen Abbruch der Ensembleaktivität durch den Schauspieler in Transkript 2 mit dem Abbruch mit Account in Transkript 3, wird 
deutlich, dass die Funktion hier im Gegensatz zum bisherigen Fokus der multiactivity-Forschung deutlich über das bloße Anzeigen eines Problems mit einer Aktivität hinausgeht. Vielmehr ermöglicht der Abbruch mit Account eine dynamische Modifikation der Teilnehmendenrahmung und damit der Fortsetzung der Aktivität, während der Abbruch ohne Account neben der potentiellen Geschichtsbedrohung auch die soziale Ordnung infrage stellt.

Tab. 5: Schema eines Aktivitätsabbruchs bei multiplen Aktivitäten.

\begin{tabular}{|c|c|c|c|}
\hline Aktivität 1 & $\begin{array}{l}\text { engagement } \\
\text { display }\end{array}$ & 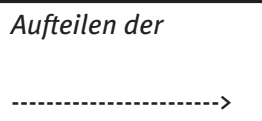 & $\begin{array}{l}\text { Abbruch bei struktureller } \\
\text { Inkompatibilität der Beteiligungsweisen } \\
-----x\end{array}$ \\
\hline Aktivität 2 & & Beteiligungsweisen & engagement display \\
\hline
\end{tabular}

Die Aktivitätsabbrüche der Fallkollektion aus den Transkripten 1, 2 und 3 funktionieren nach demselben Prinzip: In allen Ausschnitten bearbeiten die Teilnehmenden solange die relevanten Aktivitäten (z. B. durch Pausieren oder körperliche Konfiguration), bis eine der Aktivitäten in Bezug auf die Verteilung der multimodalen Beteiligungsweisen des engagement displays einen kritischen Zustand erreicht und strukturell nicht mehr mit der anderen kombinierbar ist. Dieser strukturell kritische Schwellenwert ist in den Ausschnitten 1 und 2 der Grad visueller Aufmerksamkeit, den die jeweilige LESE-Einzelaktivität zum Aufrechterhalten erfordert. In Transkript 1 wird die Einzelaktivität zugunsten der Ensembleaktivität abgebrochen, indem das Fokusobjekt der Aktivität (Smartphone) weggelegt wird, was dem Schauspieler eine verbale Beteiligung an der Aktivität ermöglicht. Der Abbruch der Ensembleaktivität ERKLÄRUNG in Transkript 2 hat nicht nur Folgen für den abbrechenden Schauspieler, der sich nun ganz auf seine LESE-Einzelaktivität des Stücktexts konzentrieren kann, sondern auch Auswirkungen auf die Teilnehmendenrahmung und die Emergenz der weiterbestehenden Aktivität. Wie der Account des Dramaturgen in Transkript 3 zeigt, bekommen Abbrüche von Ensembleaktivitäten bei gleichzeitiger Relevanz mit anderen Aktivitäten eine ganz andere interaktionale Brisanz als Abbrüche von Einzelaktivitäten. Während letztere vergleichswiese unproblematisch sind, weil sich dort die Abbrechenden nicht gegenüber anderer Beteiligter für den Abbruch rechtfertigen und sich lediglich intrapersonell koordinieren müssen, erfordert der Abbruch einer Ensembleaktivität eine Aushandlung aller an der Aktivität Beteiligten. Dies zeigt sich in Transkript 2, als der Hospitant seinen Beitrag nicht sofort mit Ausbleiben der Involvierungsanzeige des Schauspielers beendet, sondern seinen Turn trotz Disfluenzmarker pragmatisch, syntaktisch und prosodisch abschließt, 
als ein Involvierungsdisplay seines Interaktionspartners für mehrere Sekunden ausbleibt. Auch der Regisseur in Transkript 3 reagiert auf ein fehlendes Involvierungsdisplay des Interaktionspartners, indem er seine verbale Beteiligungsweise zunächst mit Disfluenzmarkern streckt, dann pausiert und erst wieder voll aufnimmt, als der Dramaturg einen (gestischen) Account für das fehlende Display geliefert hat. Damit wird sichtbar, dass Aktivitätsabbrüche nicht zwangsläufig das plötzliche Ausbleiben einer Beteiligung an einer Aktivität bedeuten, sondern geordnet im interaktionalen Prozess hergestellt werden (Tab. 5). Aktivitätsabbrüche unterscheiden sich für Ensembleaktivitäten und Einzelaktivitäten, die beide jeweils unterschiedliche Auswirkungen auf das Interaktionsensemble nach sich ziehen und unterschiedliche kommunikative Mittel zum Abbrechen erfordern, beispielsweise das Aussetzen visueller bzw. verbaler Beteiligungsweisen bei Ensembleaktivitäten oder die Reduzierung haptischer Beteiligung bei Einzelaktivitäten (sofern diese haptisch organisiert sind). Abbrüche werden damit als Praktiken der Bearbeitung gleichzeitiger Relevanz multipler Aktivitäten erkennbar, wenn sich die konkurrierenden Aktivitäten strukturell in Bezug auf die verwendeten Beteiligungsweisen nicht miteinander kombinieren lassen.

\subsubsection{Wiederaufnahmen abgebrochener Aktivitäten}

Aktivitätsabbrüche sind für Interagierende eine Möglichkeit, kritische Konkurrenzsituationen der Beteiligungsweisen ko-relevanter Aktivitäten (zum Beispiel in Hinblick auf die Verteilung multimodaler Ressourcen) bei gleichzeitiger Relevanz multipler Aktivitäten zu bearbeiten. Abgebrochene Aktivitäten können wiederaufgenommen werden, sobald die Beteiligung an einer laufenden Aktivität dies ermöglicht. Eine Wiederaufnahme (,resumption“) ist in Anlehnung an Jefferson (1972: 319-330) dadurch gekennzeichnet, dass Interagierende eine vorher nicht weiter vollzogene Beteiligungsweise wieder aufnehmen und diese als nicht zum unmittelbar vorherigen Interaktionsverlauf gehörend markieren, z. B. mithilfe von auf jeden Fall (Bührig 2002) oder maar (,aber') (Mazeland \& Huiskes 2001). Im Vergleich mit Fortsetzungen pausierter Aktivitäten („,continuations“), die in Abschnitt 5.2.2 beschrieben werden, unterscheiden sich Wiederaufnahmen abgebrochener Aktivitäten („resumptions“) in genau diesen Wiederaufnahmemarkierungen. Während Wiederaufnahmen von den Interagierenden als solche markiert werden, bedienen sich Fortsetzungen pausierter Aktivitäten sogenannter Fortsetzungsprojektionen, die anzeigen, dass die pausierte Aktivität zeitnah fortgesetzt werden wird.

Die unterschiedlichen Konsequenzen für eine Interaktion bei Wiederaufnahmen abgebrochener Einzel-im Vergleich zu Ensembleaktivitäten sollen im Folgenden anhand zweier Fälle diskutiert werden. Wie schon zuvor bei den Abbrüchen, unter- 
scheiden sich Wiederaufnahmen abgebrochener Einzelaktivitäten (Transkript 4) von Wiederaufnahmen abgebrochener Ensembleaktivitäten (Transkript 5) hinsichtlich der interaktionalen Relevanz und der Herstellung von Intersubjektivität.

Das folgende Transkript 4 zeigt eine Situation aus der Vor-Probenphase des 11. Probentages. Dort sind zunächst nur der Hospitant (HOM) und der Schauspieler (SCM) auf der Probebühne anwesend. Der Schauspieler LIEST IM NEwsFEED auf seinem Smartphone und geht damit einer Einzelaktivität via visueller und haptischer Beteiligungsweisen nach. Die Teilnehmendenrahmung ändert sich, als die Schauspielerin (SCW) gemeinsam mit der Regieassistentin (ASS) den Raum betritt und eine gemeinsame BEGRÜßUNG initiiert, an der alle Anwesenden (Schauspielerin, Schauspieler, Hospitant, Regieassistentin) per visueller und verbaler Beteiligungsweisen partizipieren (Z.001-5). Der Schauspieler, der im Rahmen der BEGRÜßUNG die Schauspielerin umarmt (Z.007-11), bricht dafür die haptische Beteiligungsweise an seiner Einzelaktivität am Smartphone ab, die er nach Abschluss der BEGRÜßUNG wieder aufnimmt (Z.014-16) und somit deutlich macht, dass diese Aktivität trotz Abbruch nach wie vor Relevanz für ihn hat.

Tab. 6: Abbruch und Wiederaufnahme der Einzelaktivität nach Abschluss der Ensembleaktivität durch SCM.

\begin{tabular}{|c|c|c|c|c|c|}
\hline Transkript 4 & & a) & & b) & \\
\hline \multirow[t]{2}{*}{$\begin{array}{l}\text { Einzelaktivi- } \\
\text { tät: LESEN }\end{array}$} & $\begin{array}{l}\text { engagement } \\
\text { display }\end{array}$ & pausieren & abbrechen & $\begin{array}{l}\text { wiederaufneh- } \\
\text { men }\end{array}$ & $\begin{array}{l}\text { engagement } \\
\text { display }\end{array}$ \\
\hline & $\begin{array}{l}\text { vis. \& hap. } \\
\text { Beteiligungsw. }\end{array}$ & $\begin{array}{l}\text { haptische } \\
\text { Beteiligungsw. }\end{array}$ & ----X & $\begin{array}{l}\text { 1. hap., } \\
\text { 2. verb. }\end{array}$ & $\begin{array}{l}\text { 3. vis. Betei- } \\
\text { ligungsweisen }\end{array}$ \\
\hline \multirow[t]{2}{*}{$\begin{array}{l}\text { Ensemble- } \\
\text { aktivität: } \\
\text { BEGRÜßEN }\end{array}$} & & $\begin{array}{l}\text { engagement } \\
\text { display }\end{array}$ & $\begin{array}{l}\text { engagement } \\
\text { display } \\
-0 .-0\end{array}$ & & \\
\hline & & $\begin{array}{l}\text { vis. \& verb. } \\
\text { Beteiligungsw. }\end{array}$ & $\begin{array}{l}\text { vis., verb. \& hap. } \\
\text { Beteiligungsw. }\end{array}$ & & \\
\hline
\end{tabular}

Die hier fokussierte Wiederaufnahme der abgebrochenen und vom Teilnehmer lokal nicht mehr bearbeiteten Einzelaktivität vollzieht der Schauspieler an der Stelle, als die Ensembleaktivität von den Teilnehmenden interaktional beendet wird. Der Schauspieler muss somit das engagement display bzgl. der Ensembleaktivität nicht mehr aufrechterhalten und kann die freiwerdenden multimodalen Ressourcen (wieder) auf das engagement display der Einzelaktivität übertragen (Tab. 6). 


\section{Transkript 4: Wiederaufnahme der abgebrochenen Einzelaktivität IM NEWSFEED}

\section{LESEN nach Abschluss der Ensembleaktivität BEgRüßUNG}

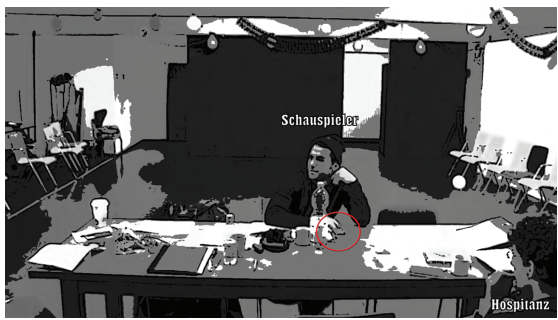

Abb. 4.1: SCM hält Hand am Smartphone.

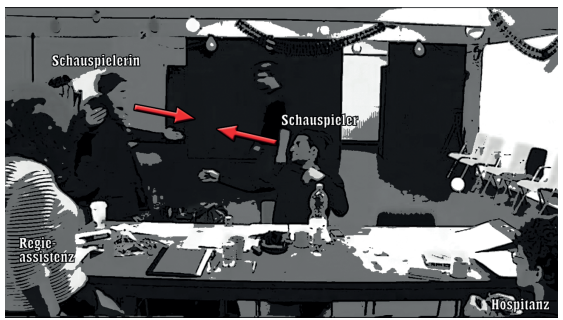

Abb. 4.2: SCW und SCM umarmen sich.

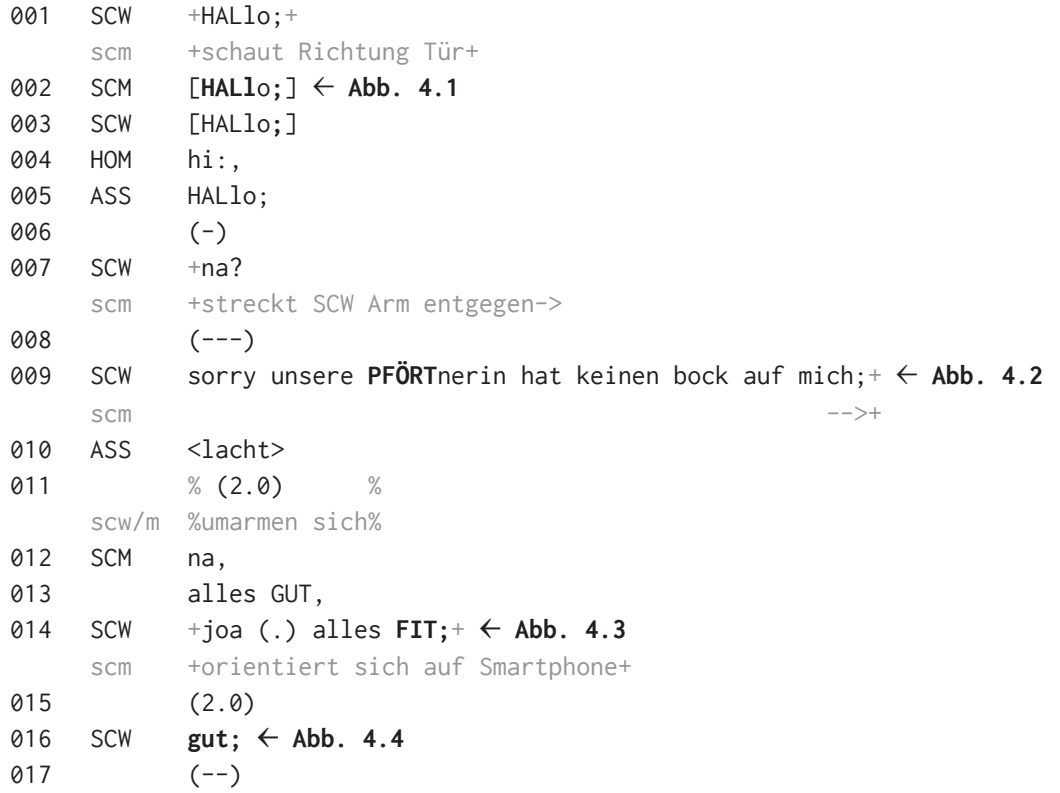

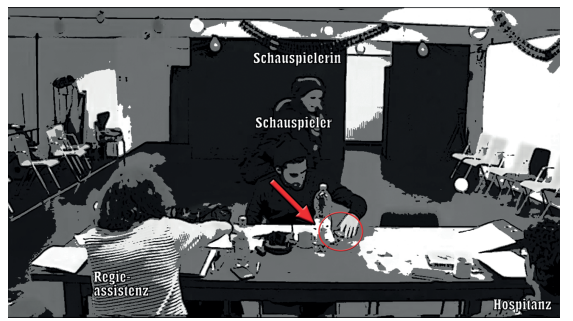

Abb. 4.3: SCM zieht Smartphone zu sich heran.

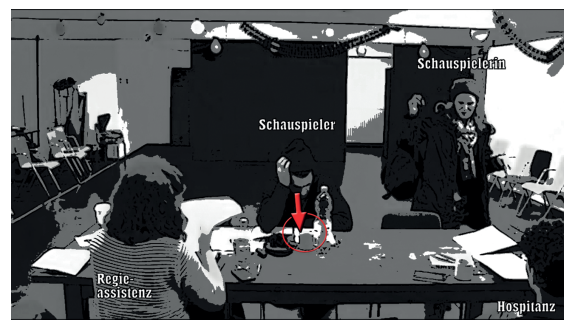

Abb. 4.4: SCM nimmt LESE-Einzelaktivität wieder auf. 
a) Abbruch der Einzelaktivität durch Auflösen des engagement displays (Z.001-011): Der Abbruch der Smartphone-Einzelaktivität IM NEWSFEED LESEN gestaltet sich nach dem gleichen Muster wie bereits in Transkript 1. Der Schauspieler teilt die beiden Beteiligungsweisen, die er für die Einzelaktivität verwendet, auf die beiden gleichzeitig relevanten Aktivitäten auf, sodass er sich verbal und visuell an der Ensembleaktivität beteiligen kann, während er immer noch die haptische Anforderung der Einzelaktivität erfüllt. Als der Schauspieler den ersten Paarteil der BEGRÜßUNG durch die Schauspielerin wahrnimmt, löst er seine visuelle Beteiligungsweise an der Einzelaktivität auf und monitort den Verlauf der Ensembleaktivität (Z.001), an der er sich beteiligt, indem er mit einem Gegengruß die konditionelle Relevanz einlöst (Z.002). Währenddessen verharrt sein Zeigefinger, den er zum Navigieren im Newsfeed verwendet, auf dem Display des Smartphones (Abb. 4.1) und hält seine Involvierung in diese Aktivität (im pausierten Zustand, vgl. 5.2.2) aufrecht. Dies ändert sich, als er gleichzeitig mit der Realisierung der Fragepartikel „na?“ durch die Schauspielerin (Z.007) seinen Arm hebt und ihr damit eine Umarmung anbietet (Abb. 4.2). Anders als in Transkript 1, in dem ihm die Hand angeboten wurde, ist es hier der Schauspieler, der seiner Interaktionspartnerin eine körperlich ausgebaute BEGRÜßUNG (vgl. Goodwin 2021) anbietet und die Einzelaktivität damit von sich aus in den Hintergrund verschiebt, da er im Zuge dessen seine Involvierung in seine Einzelaktivität abbricht. Der Abbruch ist an dieser Stelle die notwendige Konsequenz seiner Beteiligung an der BEGRÜßUNG, die, wie schon in Transkript 1, von einer visuellen (Monitoring), über eine verbale $(\mathrm{Gru} \beta) \mathrm{zu}$ einer haptisch-interkorporalen Beteiligung (Händeschütteln bzw. Umarmung) semiotisch erweitert wird und dadurch an Komplexität gewinnt. Mit zunehmender Komplexität der Beteiligung geht der gesteigerte Bedarf an multimodalen Ressourcen einher. Dies äußert sich hier darin, dass, je komplexer die Beteiligung an der Ensembleaktivität wird, die multimodale Beteiligung an der Einzelaktivität abnimmt, bis diese nicht mehr aufrechterhalten werden kann und abgebrochen wird.

b) Wiederaufnahme der abgebrochenen Einzelaktivität nach Abschluss der Ensembleaktivität (Z.012-017): Der semiotisch geordnete Prozess zeigt sich in derselben Weise nicht nur beim Fall des Abbruchs, sondern auch bei der Wiederaufnahme. Einzig die Reihenfolge, in der sich die multimodale Komplexität der Beteiligung verändert, ist in diesem Fall gegenläufig: Noch während die Schauspielerin die ihr vom Schauspieler gestellte Frage nach ihrem Wohlbefinden (Z.012-3) bearbeitet (Z.014), reorientiert sich der Schauspieler wieder auf sein Smartphone, das er sich zu sich heranzieht (Abb. 4.3). Die Schauspielerin bearbeitet damit an dieser Stelle eine Interaktionsaufgabe einer Aktivität, an der der Schauspieler keine Beteiligung mehr anzeigt. Die darauffolgenden sprachenfreien zwei Sekunden 
tragen diesem Umstand seiner ausbleibenden Beteiligung Rechnung. Es erfolgt daraufhin (analog zu Transkript 2) keine weitere Beteiligung an dieser Ensembleaktivität durch die Teilnehmenden, die damit mit dem Einlösen der konditionellen Relevanz der Frage nach dem Wohlbefinden von den Teilnehmenden als abgeschlossen behandelt wird. Die Wiederaufnahme der LESE-Aktivität des Schauspielers erfolgt demnach mit dem Abschluss der Ensembleaktivität. Er löst dazu zuerst die Umarmung (haptische Beteiligungsweise), stellt dann seine verbale Beteiligung ein und verändert zum Abschluss sein visuelles MonitoringVerhalten von seiner Interaktionspartnerin auf das Smartphone als Fokusobjekt seiner Einzelaktivität (Abb. 4.4).

Anhand des Abbruchs und der Wiederaufnahme einer Einzelaktivität nach Abschluss einer Ensembleaktivität lassen sich in diesem Beispiel die fortbestehenden Relevanzen von Aktivitäten beobachten. Der Schauspieler reagiert mit seinem Abbruch auf die unterschiedlichen Komplexitäten in der multimodalen Beteiligung bei simultan relevanten Aktivitäten, was in seinem engagement display sichtbar wird. Da BEGRÜßEN und LESEN in der analysierten Situation hinsichtlich der visuellen Beteiligungsweise, die nicht aufgeteilt werden kann, strukturell inkompatibel sind (vgl. Kap. 7), steht der Schauspieler vor der Wahl eine der beiden Aktivitäten zugunsten der anderen zu priorisieren. Da eine Weiterbearbeitung der Einzelaktivität dadurch ein disengagement display bzgl. der Ensembleaktivität bedeuten würde, entscheidet er sich hier für die intersubjektiv hergestellte Ensembleaktivität, bricht die Einzelaktivität ab und verschiebt ihre Relevanz somit in den Hintergrund. Indem er diese Aktivität durch die nicht explizit intersubjektiv ausgehandelte Wiederaufnahme wieder in den Vordergrund holt, löst er seine Beteiligung an der Ensembleaktivität auf und zeigt damit seine Interpretation dieser Aktivität als lokal weniger relevant an. Für das Interaktionsensemble zieht dies die Auflösung der Ensembleaktivität nach sich.

Im Vergleich dazu gestalten sich Wiederaufnahmen abgebrochener Ensembleaktivitäten deutlich komplexer, da die Wiederaufnahme dabei einer Aushandlung durch das Interaktionsensemble bedarf. In folgendem Transkriptausschnitt (Transkript 5) berichtet der Regisseur (REG) den Anwesenden (unter anderem Schauspielerin (SCW), Ausstatterin (AUS) und Praktikantin (PRW)) von seinem Treffen mit einer Mutter und ihrem Sohn, der eine kleine Rolle in dem Stück übernehmen soll. Der Ausschnitt setzt ein, als der Regisseur den (abwesenden) Jungen VORSTELLT. Der Regisseur bricht im Verlauf der untenstehenden Sequenz diese Ensembleaktivität zugunsten einer vorher nicht thematisierten Einzelaktivität ab, die er simultan bearbeitet: das SuCHEN EINEs MusIKvideos am Tablet. Beide Aktivitäten vollzieht er dabei einerseits unter Rückgriff auf visuelle Beteiligungsweisen und andererseits mithilfe von verbalen (nur bei der Ensembleaktivität) und hapti- 
schen Beteiligungsweisen (nur bei der Einzelaktivität). Damit steht der Regisseur wie die Teilnehmenden der bisher besprochenen Beispiele vor der Herausforderung, zwei strukturell inkompatible Aktivitäten zu koordinieren. Der Regisseur löst dieses Problem, indem er die Ensembleaktivität a) kurzzeitig abbricht und sie b) nach erfolgreichem Abschluss der Einzelaktivität wieder aufnimmt, als er dem Interaktionsensemble das Ergebnis der SucHE präsentiert. Im Unterschied zu den bisher diskutierten Fällen hat der Regisseur hier bei beiden Aktivitäten (VoRSTELLEN und MUSIKVIDEO SUCHEN) den Partizipationsstatus principal (Goffman 1979) inne: Er ist sowohl derjenige, der autonom das Tablet bedient als auch derjenige, der den Jungen mit seiner Mutter getroffen hat und somit die epistemische Autorität (Heritage 2012) bezüglich dieses Erlebnisses hat. Wie die folgende Analyse zeigt, hat dies unmittelbar Auswirkungen auf die Organisation des Interaktionsensembles während des Abbruchs und die intersubjektive Gestaltung der Wiederaufnahme der abgebrochenen Ensembleaktivität.

Der Partizipationsstatus des Regisseurs als principal beider ko-relevanter Aktivitäten wird anhand des koordinativen Verhaltens der anderen Beteiligten deutlich. Im Rahmen der Orientierungen der Ko-Interagierenden wird sichtbar, dass die meisten Mitglieder des Interaktionsensembles das engagement display bzgl. der Einzelaktivität MusIKVIDEO SUCHEN seitens des Regisseurs als disengagement display bzgl. der Ensembleaktivität VoRSTELLEN werten.

Tab. 7: Abbruch und Wiederaufnahme der Ensembleaktivität nach Abschluss der Einzelaktivität durch REG.

\begin{tabular}{|c|c|c|c|c|c|c|}
\hline Transkript 5 & & & a) & & & b) \\
\hline $\begin{array}{l}\text { Einzelaktivi- } \\
\text { tät: MUSIKVI- } \\
\text { DEO SUCHEN }\end{array}$ & & \begin{tabular}{l} 
engag \\
display \\
\hdashline$-{ }^{2}$ \\
vis. \& hap. \\
Beteili- \\
gungsw.
\end{tabular} & $\begin{array}{l}\text { engag } \\
\text { display } \\
- \text { vis. \& hap. } \\
\text { Beteili- } \\
\text { gungsw. }\end{array}$ & 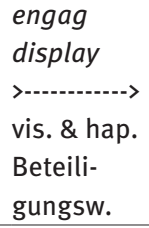 & \begin{tabular}{l} 
engag \\
display \\
- \hdashline$-{ }^{-}$vis. \& hap. \\
Beteili- \\
gungsw.
\end{tabular} & \\
\hline $\begin{array}{l}\text { Ensembleak- } \\
\text { tivität: } \\
\text { voRSTELLEN }\end{array}$ & $\begin{array}{l}\text { engag. display } \\
\text {------> } \\
\text { vis. \& verb. } \\
\text { Beteiligungsw. }\end{array}$ & pausieren & $\begin{array}{l}\text { engag display } \\
\text { >---:---> } \\
\text { verbale } \\
\text { Beteili- } \\
\text { gungsw. }\end{array}$ & $\begin{array}{l}\text { abbrechen } \\
-----x\end{array}$ & & $\begin{array}{l}\text { wiederauf- } \\
\text { nehmen } \\
>--------> \\
\text { vis. \& verb. } \\
\text { Beteili- } \\
\text { gungsw. }\end{array}$ \\
\hline
\end{tabular}

Da keiner der Beteiligten über die epistemische Autorität verfügt das VoRSTELLEN weiterzubearbeiten, wenden sie sich als Folge visuell vom Regisseur als Initiator der Ensembleaktivität ab und behandeln die Ensembleaktivität VoRSTELLEN somit 
als abgebrochen. Die Wiederaufnahme der Ensembleaktivität nach Abschluss der Einzelaktivität hängt abermals mit dem Partizipationsstatus des Regisseurs als principal der ko-relevanten Aktivitäten zusammen. Erst wenn der Regisseur wieder ein engagement display für die Ensembleaktivität anzeigt, nehmen auch die übrigen Mitglieder des Interaktionsensembles ihre Beteiligungsweisen an der Ensembleaktivität wieder auf (Tab. 7).

\section{Transkript 5: Wiederaufnahme der abgebrochenen Ensembleaktivität VoRSTELLEN DES NEUEn ENSEMBLEMITGLIEDS nach Abschluss der Einzelaktivität MUSIKVIDEO SUCHEN}

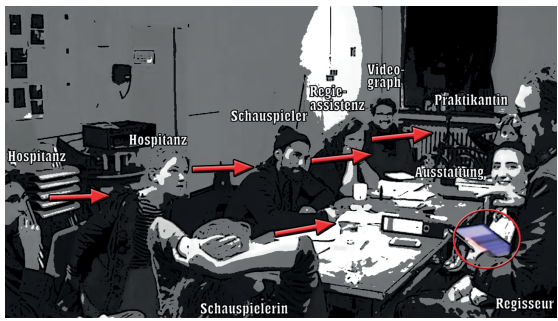

Abb. 5.1: Alle Anwesenden schauen REG an.

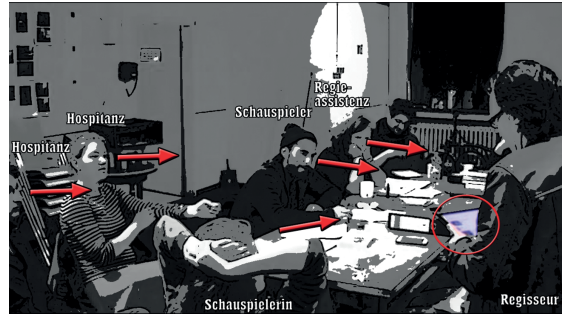

Abb. 5.2: REG scrollt durch Videofeed.

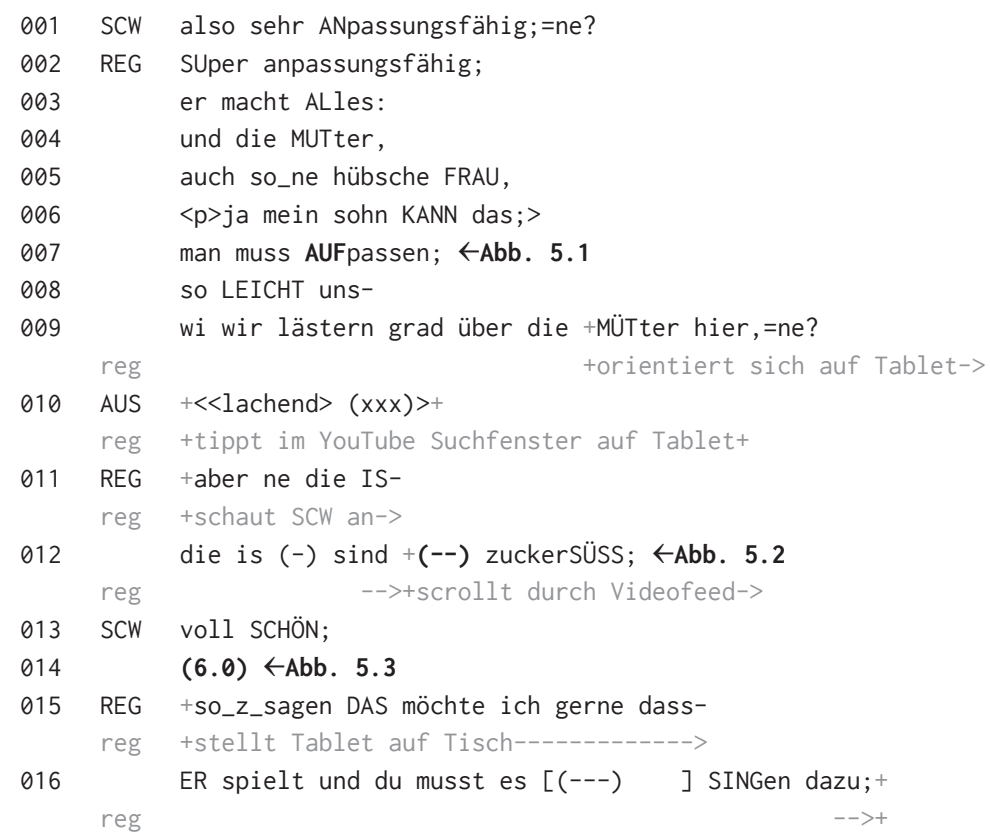




\begin{tabular}{|c|c|c|}
\hline 017 & $\mathrm{SCW}$ & [oh NEIN; ] \\
\hline 018 & & $*(6.0) *$ \\
\hline & all & *arrangieren sich um Tablet herum* \\
\hline 019 & REG & das geht um ras[SISmus; ] $\leftarrow$ Abb. $\mathbf{5 . 4}$ \\
\hline 20 & PRW & [KENN ich;] \\
\hline
\end{tabular}

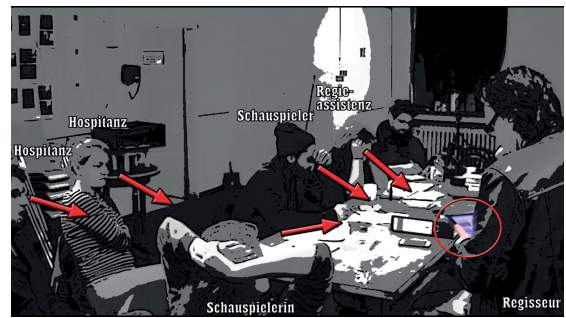

Abb. 5.3: SCW verfolgt REGs Tätigkeit am Tablet.

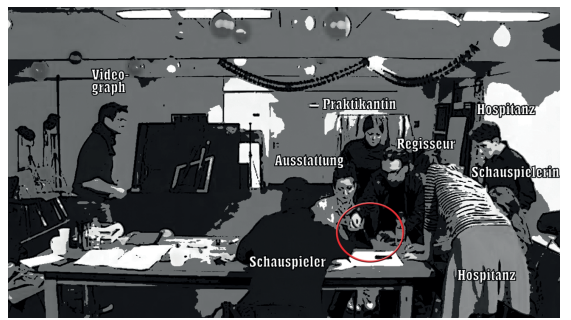

Abb. 5.4: Die Beteiligten arrangieren sich um das Tablet.

a) Abbruch der Ensembleaktivität zugunsten der Einzelaktivität (Z.001-013): Während der Ensembleaktivität (Z.001-9), die der Regisseur selber als „lästern“ (Z.009) beschreibt (Günthner \& Christmann 1996; Günthner 2013), sind alle Anwesenden visuell auf den Regisseur orientiert (Abb. 5.1). Diese Orientierung bleibt bestehen, als der Regisseur sich während seines Turns seinem Tablet zuwendet (Z.009) und etwas in das YouTube-Suchfenster eintippt (Z.010). Er pausiert diese haptische und visuelle Beteiligung an seiner Einzelaktivität MUSIKVIDEO SUCHEN kurzzeitig, um eine Einschränkung bzgl. seines Lästerturns an die Schauspielerin zu adressieren (Z.011-12). Diesen Interaktionsbeitrag gestaltet der Regisseur so, dass er ihn als duales engagement display bzgl. der beiden gleichzeitig relevanten Aktivitäten organisieren kann. Dazu vollzieht er den ersten Teil der Äußerung inklusive Selbstreparatur (,die is (-) sind“, Z.012) mit auf die Adressatin ausgerichtetem Blick. Den zweiten Teil der verbalen Beteiligung („zuckerSÜSS“, Z.012), bei dem er die projizierte Leerstelle einer Bewertung einlöst, vollzieht er nach einer verbalen Pause, in der er seinen Blick von der Schauspielerin ab- und dem Tablet zuwendet, um dort per haptischer Beteiligungsweise seine Einzelaktivität MusıKVIDEO SUCHEN zu bearbeiten. Damit beteiligt sich der Regisseur im Rahmen dieses Interaktionsbeitrages gleichzeitig verbal an der Ensembleaktivität, während er per visueller und haptischer Beteiligungsweisen ein engagement display bzgl. der Einzelaktivität anzeigt. Indes der Regisseur durch den Videofeed scrollt und dabei immer längere verbale Pausen produziert, liefert die Schauspielerin per verbaler Beteiligung in Form einer positiven Bewertung (,voll schön“, Z.013) einen Hinweis auf ihre Interpretation seiner fortbestehenden Involvierung in die Ensembleaktivität. Die anderen Mitglieder des Interaktionsensembles bleiben weiterhin visuell 
auf den Regisseur orientiert und zeigen somit ebenfalls ihr Verständnis seines weiterhin aktiven engagement display bzgl. der Ensembleaktivität an (Abb. 5.2).

\section{b) Wiederaufnahme der Ensembleaktivität nach Abschluss der Einzelaktivität} (Z.014-020): Diese Orientierung des Interaktionsensembles auf den Regisseur ändert sich, als der Regisseur sich im weiten Verlauf weder visuell noch verbal an der Ensembleaktivität beteiligt. Mit Ausnahme der Schauspielerin, die von ihrem Sitzplatz aus das Tabletdisplay des Regisseurs monitoren kann, ist für keine*n der anderen Beteiligten transparent, was der Regisseur am Tablet macht. Als Konsequenz dieser nicht-intersubjektiven Gestaltung der Einzelaktivität MusıKVIDEO SUCHEN lösen - mit Ausnahme der Schauspielerin - alle Teilnehmenden ihre visuelle Ausrichtung auf den Regisseur auf, als dieser für sechs Sekunden keine Beteiligung an der Ensembleaktivität anzeigt (Z.14, Abb. 5.3). Ohne verbale oder visuelle Beteiligungsweise des Regisseurs behandeln die Teilnehmenden die Ensembleaktivität damit als situativ nicht mehr relevant und orientieren sich anderweitig bzw. nehmen eigene Einzelaktivitäten auf (z. B. LESEN bei Schauspieler und Regieassistentin). Erst als der Regisseur das Tablet auf den Tisch stellt (=visueller Abschlussmarker) und die Schauspielerin darüber informiert, sie müsse „SINGen dazu“ (Z.016), wird deutlich, dass er ein bestimmtes MUSIKVIDEO GESUCHT hat, das er nun gefunden hat (=Abschluss der Einzelaktivität) und den Anwesenden vorstellen wird. Diese nehmen ihre Beteiligungen an der Ensembleaktivität wieder auf und arrangieren sich so um das Tablet, dass alle das Musikvideo sehen können (Abb. 5.4). Der Regisseur markiert die Wiederaufnahme der Ensembleaktivität dadurch, dass er die Kohärenz sprachlich wiederherstellt. Dazu rahmt er die Wiederaufnahme mithilfe der Konnektivpartikel (vgl. Bührig 2009) sozusagen als Teil eines argumentativen Diskurses (,so_z_sagen DAS möchte ich gerne dass“, Z.015), den er dadurch an die vorausgegangenen Interaktionsbeiträge anbindet. Die Konnektivpartikel sozusagen in Verbindung mit der anschließenden Erklärung, was der Regisseur von der Schauspielerin verlangt, verhält sich hier funktional ähnlich zu jedenfalls und aufjeden Fall (Bührig 2002), die ebenfalls die retrospektive Anbindung an einen vorherigen Interaktionsbeitrag leisten. Die vom Regisseur verwendete Konnektivpartikel sozusagen ähnelt in ihrer syntaktischen und sequentiellen Position den Partikeln jedenfalls/auf jeden Fall. Sie steht vor dem finiten Verb bzw. nach Abschluss einer Sequenz, die nicht zur vorherigen Sequenz gehört hat und markiert damit globale Kohärenz $^{30}$ (Lenk 1998) zweier zusammengehörender Sequenzen, die von einer

30 Lenk (1998: 27) definiert globale Koherenz wie folgt: „global coherence relations are the relations between segments in discourse that appear further apart, with other stretches of discourse inbetween.” 
dritten Sequenz unterbrochen worden sind (side sequences, Jefferson 1972). Die wiedereinsetzende Sprachproduktion des Regisseurs wird dadurch für die Teilnehmenden erkennbar als etwas, das zu seiner vorherigen Beteiligungsweise an der Ensembleaktivität gehört und nicht als Beteiligungsweise an einer neuen Aktivität. Dies wird umso deutlicher, wenn der Regisseur in der Folge-TCU das Personalpronomen er (Z.016) verwendet um auf den Jungen zu referieren, statt ihn noch mal einzuführen. Dieser Kohäsionsindikator zeigt den anderen Mitgliedern des Interaktionsensembles als weiteres Indiz an, dass der Turn thematisch an die vorausgegangenen Interaktionsbeiträge anschließt (und nicht etwa eine neue Sequenz beginnt) und so die zuvor abgebrochene Ensembleaktivität wieder relevant gesetzt wird. Diese Interpretation zeigt sich in der Reaktion der anderen Teilnehmenden, die die wiederaufgenommene Ensembleaktivität als Fortsetzung des vorherigen Diskurses behandeln, indem sie ihre Einzelaktivitäten beenden und sich sowohl verbal (Z.017 \& Z.020) als auch mithilfe ihrer Körperarrangements (Abb. 5.4) an der wieder relevanten Ensembleaktivität beteiligen.

Wie schon in den Transkripten 2 und 3 zieht auch hier der Beteiligungsrückzug an einer Ensembleaktivität das Ende bzw. eine Pausierung einer Beteiligungsweise dieser Ensembleaktivität nach sich. Parallel zum Fall in Transkript 3 liefert der abbrechende Interagierende hier einen Account, der die emergente Modifikation der Ensembleaktivität ermöglicht. Anders als in Transkript 3 wird dieser Account jedoch nicht mit dem Abbruch, sondern erst bei der Wiederaufnahme mithilfe des visuellen Abschlussmarkers Tablet auf Tisch und einer Konnektivpartikel mitsamt anschließender Erläuterung geliefert, was in den Wiederaufnahmen der Beteiligungsweisen durch die Mitglieder des Interaktionsensembles erkennbar wird. Damit wird der Abbruch retrospektiv erklärbar und ermöglicht prospektiv eine Orientierung auf diese wieder relevant gesetzte Ensembleaktivität. Die schematische Darstellung der temporalen Verfahren dieses Falls in der obenstehenden Tab. 7 zeigen deutlich die alternierende Struktur zwischen pausierter Beteiligung und vollem engagement display. Diese Struktur gibt der Regisseur auf, indem er seine Beteiligung an der Ensembleaktivität abbricht und diese erst wieder aufnimmt, als die Einzelaktivität abgeschlossen ist.

Interagierende können mit diesen Praktiken den Anforderungen multipler Aktivitäten begegnen, indem sie auf eine veränderte Relevanz der Aktivitäten für den ${ }^{\star}$ die koordinierende Interagierende`n oder auf strukturell konkurrierende multimodale Ressourcen verweisen. Bereits damit wird deutlich, dass die Koordinationspraktiken Abbrechen und Wiederaufnehmen von Aktivitäten zur Bearbeitung gleichzeitiger Relevanz bei strukturell inkompatiblen engagement displays keine reinen temporalen Phänomene darstellen, sondern den Beteiligten eine Möglichkeit eröffnen, ihre Interpretation des laufenden Geschehens anzuzeigen. Im Gegen- 
satz zu interaktional ausgehandelten Aktivitätsabschlüssen (Abschluss der BEGRÜßUNG zwischen Schauspielerin und Schauspieler in Transkript 4) entsteht bei einem Aktivitätsabbruch eine Bruchkante. Eine solche Bruchkante zeigt sich am stärksten in der intersubjektiven Rahmung einer Aktivität, wenn Abbrüche eine potentielle Gesichtsbedrohung für Ko-Interagierende nach sich ziehen können (Transkript 2) oder eine Veränderung des Adressentenzuschnitts zur Folge haben (Transkript 3). Abgebrochene Aktivitäten zeichnen sich dadurch aus, dass sie kurzzeitig nicht bearbeitet werden und auch kein Element auf eine zeitnahe Wiederaufnahme hinweist. ${ }^{31}$ Soll eine abgebrochene Aktivität wiederaufgenommen werden, kann diese Wiederaufnahme bei einer Ensembleaktivität intersubjektiv gestaltet werden (z. B. mithilfe einer Konnektivpartikel) oder bei Einzelaktivitäten auch autonom vollzogen werden (Transkript 4 für Wiederaufnahmen der Einzelaktivität und Transkript 5 für Wiederaufnahmen der Ensembleaktivität). In beiden Fällen greifen Interagierende bei wiederaufgenommenen Aktivitäten auf jene Beteiligungsweisen zurück, die auch vor dem Abbruch zum Einsatz kamen. Abbrüche von Einzelaktivitäten zugunsten von Ensembleaktivitäten organisieren die Interagierenden in den Transkripten 1 und 4, indem sie die visuelle Orientierung und die haptische Bindung mit dem Fokusobjekt Smartphone der Einzelaktivitäten auflösen und im Gegenzug die Beteiligung an der Ensembleaktivität als engagement display organisieren. Abbrüche von Ensembleaktivitäten zugunsten von Einzelaktivitäten werden von den Interagierenden in den besprochenen Ausschnitten hergestellt, indem sie die verbale und visuelle Orientierung mit den Ko-Interagierenden der Ensembleaktivität aufheben (Transkript 2), Nichtverfügbarkeit mithilfe eines Accounts anzeigen (Transkript 3) oder das engagement display einer Einzelaktivität relevant setzen (Transkript 5). Einzel- und Ensembleaktivitäten unterscheiden sich dahingehend systematisch voneinander, dass Einzelaktivitäten ohne Account abgebrochen und wiederaufgenommen werden können, wohingegen es für die intersubjektive Struktur der Ensembleaktivitäten von großer Bedeutung ist, ob und wie die Abbrüche und Wiederaufnahmen accountable gemacht werden. Durch Aktivitätsabbrüche und -wiederaufnahmen entsteht eine Aktivitätsserie, die dadurch gekennzeichnet ist, dass Teilnehmende immer nur ein engagement display bzgl. einer der gleichzeitig relevanten Aktivitäten anzeigen und die jeweils andere Aktivität für die Dauer der Bearbeitung zurückstellen (Tab. 8).

31 Im Gegensatz dazu handelt es sich bei einer pausierten Aktivität um einen Handlungsverlauf, der zwar nicht aktiv bearbeitet wird, aber mindestens ein Element einer Beteiligungsweise beinhaltet, welche die fortbestehende Involvierung in diese Aktivität anzeigt (siehe 5.2). Das solche pausierten Aktivitäten interaktional in Abbrüche überführt werden können, zeigen bereits die Transkripte 1, 2, 3 \& 5 . 
Tab. 8: Schema Aktivitätsabbruch mit Wiederaufnahme bei Aktivitätsabschluss bei multiplen Aktivitäten.

\begin{tabular}{|c|c|c|c|c|c|}
\hline Aktivität 1 & $\begin{array}{l}\text { engag. } \\
\text { display }\end{array}$ & Aufteilen der & $\begin{array}{l}\text { Abbruch bei } \\
\text { struk. Inkompa. } \\
\text { d. Beteiligungsw. }\end{array}$ & Wiederaufnahme & $\begin{array}{l}\text { engag. } \\
\text { display }\end{array}$ \\
\hline & --.--.--..- & 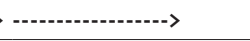 & -----------x & >--------.> & 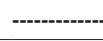 \\
\hline Aktivität 2 & & Beteiligungsweisen & engag. display & Abschluss & \\
\hline
\end{tabular}

Dieses Zurückstellen leisten die Interagierenden in den besprochenen Fällen, indem sie eine zentrale Beteiligungsweise der konkurrierenden Aktivität pausieren, bevor sie abgebrochen wird. Dies realisieren sie beispielsweise dadurch, dass sie die haptische Bindung mit einem Fokusobjekt beibehalten oder den Blickkontakt aufrechterhalten, während die verbalen Beteiligungsweisen an Ensembleaktivitäten pausiert werden. Erst wenn die Pausierung hinsichtlich der verwendeten Beteiligungsweisen einen kritischen Status erreicht und nicht länger aufrechterhalten werden kann - beispielsweise, wenn die Bearbeitung der ko-relevanten Aktivität die Beteiligungsweise der pausierten Aktivität benötigt -, wird die Pausierung in einen Abbruch überführt. Das Pausieren einer Aktivität zugunsten einer anderen ist damit ein Verfahren, das über die serielle Koordination hinausgeht. Denn im Gegensatz zum Aktivitätsabbruch wird beim Aktivitätspausieren die Aktivität nicht zu einem (vorläufigen) Ende gebracht. Stattdessen bleibt die Involvierung bestehen, auch wenn sich die Beteiligung an dieser Aktivität auf das Aufrechterhalten beschränkt. Sind Abbrüche und Wiederaufnahmen Praktiken des entweder oder, dann ermöglichen Aktivitätspausierungen engagement displays des sowohl als auch. Praktiken, die solche (quasi-)simultane Koordination multipler Aktivitäten ermöglichen, werden im nächsten Abschnitt beschrieben.

\subsection{Praktiken der quasi-simultanen Koordination: Pausieren und Fortsetzen}

Im Unterschied zur seriellen Koordination (5.1) greifen Beteiligte auf Praktiken der quasi-simultanen Koordination zurück, wenn eine Aktivität zwar weiterhin aktiv gehalten werden soll, aber eine konkurrierende Aktivität kurzzeitig besondere Relevanz bekommt. Interagierende bearbeiten eine solche Relevantsetzung dadurch, dass sie die jeweils andere Aktivität pausieren (5.2.1). Interagierende pausieren eine gleichzeitig relevante Aktivität, indem sie deren vordergründige Relevanz nicht bearbeiten, aber dennoch mindestens eine Beteiligungsweise auf 
diese Aktivität ausrichten. Diese Beteiligungswiese wird typischerweise für die Dauer der Pausierung in der Start- (Transkript 6 \& 7), Kern- (Transkript 8 \& 9) oder Endphase (Transkript 10) der jeweiligen Aktivität eingefroren. Die Art der eingefrorenen Beteiligungsweise und der Zeitpunkt der Pausierung haben dabei Konsequenzen für die ko-relevanten Aktivitäten. Wenig überraschend unterscheidet sich das Aktivitätspausieren systematisch für Einzelaktivitäten und Ensembleaktivitäten aufgrund ihrer verschiedenen intersubjektiven Organisation. Dies soll zunächst mithilfe einer Kollektion aus drei Fällen gezeigt werden. Einzelaktivitäten im Rahmen gleichzeitiger Relevanz zu pausieren, kann dabei eine Reaktion der Interagierenden auf solche situative Anforderungen darstellen, bei der sie mithilfe des Pausierens sicherstellen, dass sie ihre multimodalen Ressourcen so organisieren können, dass eine Beteiligung an den bzw. eine Bearbeitung der jeweils relevanten Aktivitäten möglich wird (Transkript 6). Darüber hinaus ist das Pausieren einer Einzelaktivität eine Praktik, mit der Interagierende auf sich verändernde Teilnehmendenrahmungen reagieren (Transkript 7), während im Gegenzug pausierte Ensembleaktivitäten den Grund für Veränderungen im participation framework darstellen können (Transkript 8). Pausierte Aktivitäten können aus dem pausierten Zustand reaktiviert und an interaktional relevanten Stellen fortgesetzt werden (5.2.2), wenn Interagierende den aufrechterhaltenen Aktivitäten einen abgeschlossenen Zustand zuschreiben, z. B. wenn eine Ensembleaktivität abgebrochen (Transkript 9), die konditionelle Relevanz eingelöst (Transkript 10) oder eine Handlungssequenz gemeinsam zu Ende gebracht wurde (Transkript 11). Wiederaufnahmen und Fortsetzungen von Aktivitäten, die im Rahmen gleichzeitiger Relevanz abgebrochen bzw. pausiert wurden, unterscheiden sich hinsichtlich der Ausprägung des Zurückstellens der Aktivitäten. Dies zeigt sich im engagement display anhand der Fortsetzungsprojektion. Während Abbrüche dadurch als solche erkennbar werden, dass es keine situative Projektion einer Fortsetzung gibt und die Wiederaufnahme bei intersubjektiv gestalteten Aktivitäten interaktional markiert werden muss, sind durchgängige Fortsetzungsprojektionen zentraler Teil pausierter Aktivitäten.

\subsubsection{Aktivitätspausieren bei simultaner Beteiligung an einer anderen Aktivität}

Im Gegensatz zu Abbrüchen multipler Aktivitäten zeichnen sich Pausierungen dadurch aus, dass die zurückgestellte Aktivität mit mindestens einer Beteiligungsweise weiterhin relevant gehalten wird - beispielsweise durch das Aufrechterhalten einer haptischen Bindung oder einer fortgesetzten visuellen Orientierung. Es muss also in jedem Fall eine konstante Involvierung in eine solche pausierte Aktivität angezeigt werden, was mit dem Einsatz einiger multimodaler Ressourcen ein- 
hergeht. Wie im Folgenden deutlich wird, „lohnen“ sich pausierte Aktivitäten für die Interagierenden vor allem dann, wenn eine Fortsetzung der jeweiligen Aktivität absehbar ist und damit eine bestehende gleichzeitige Relevanz bearbeitet werden kann. ${ }^{32}$ Aktivitäten in Situationen mit multiplen Aktivitäten werden dann pausiert, wenn die konkurrierende Aktivität eine Beteiligungsweise erfordert, die auch Bestandteil einer anderen, laufenden Aktivität ist. Deutlich wird dies an folgendem Ausschnitt. Dieser zeigt eine Sequenz vom zweiten Tag der Produktion, an dem Ideen besprochen und Textmaterial zusammengetragen wird. Die Schauspielerin (SCW) hat im Anschluss an die erste Probe nach Liedern gesucht, die die Position ihrer Figur betonen und im Stück verwendet werden könnten.

Die Schauspielerin wendet sich im folgenden Transkript im Rahmen eines VoRSCHLAGS an den Regisseur und bietet ihm ein Lied zur Verwendung im Theaterstück an. VORSCHLÄGE werden in der konversationsanalytischen Forschungslandschaft bislang unter den Gesichtspunkten der gemeinsamen Herstellung von Übereinkünften (Houtkoop-Steenstra 1987, 1990; Stevanovic \& Peräkylä 2012), KostenNutzen-Verteilungen (Couper-Kuhlen 2014), Elizitieren von Unterstützung und neuen Ensembleaktivitäten (Kendrick \& Drew 2016; Stivers \& Sidnell 2016) sowie Modifikationen von VoRSCHLÄGEN in Mensch-Maschine-Interaktionen (Opfermann \& Pitsch 2017; Opfermann et al. 2017) betrachtet. Die zentrale Gemeinsamkeit all dieser Studien ist die sequentielle Organisation von VoRSCHLÄGEN, die im Kern stets als Paarsequenz aus Angeboten und Annahmen oder Ablehnungen hergestellt werden. Dabei projiziert die erste Handlung eine bestimmte Reaktion und weist eventuell je nach Situationszuschnitt eine inhärente Präferenzorganisation auf. VORSCHLÄGE im Rahmen multipler Aktivitäten wurden bislang noch nicht erforscht.

Am Morgen des zweiten Probentages holt die Schauspielerin (SCW) ihr Tablet heraus, um dem Regisseur ein recherchiertes Lied vorzuspielen und damit für das Stück vorzuschlagen (Z.001-4). Der Regisseur (REG) hat allerdings andere Vorstellungen und bietet einen GEGENVORSCHLAG an (Z.005-16). Während die beiden Interagierenden gemeinsam die Ensembleaktivität VorSCHLAG herstellen, sind sie zudem jeweils noch in eine Einzelaktivität involviert, die sie mit der Ensembleaktivität koordinieren: Die Schauspielerin ZIEHT IHRE JACKE AUS, während der Regisseur seine Tasche AUSPACKT. Im Rahmen der verbalen Beteiligung an der Ensembleaktivität VoRSCHLAG pausiert der Regisseur seine Einzelaktivität AusPACKEN, die er erst mit dem Ende seiner verbalen Beteiligung an der

32 Ist abzusehen, dass die pausierte Aktivität nicht zeitnah fortgesetzt werden wird oder die zur Pausierung notwendigen Beteiligungsweisen anderweitig gebraucht werden, wird die Pausierung in einen Abbruch überführt (siehe z. B. Transkript 1). 
Ensembleaktivität wieder aufnimmt. Der Regisseur beteiligt sich an den Aktivitäten AuSPACKEN und Vorschlagen jeweils unter Rückgriff auf visuelle Beteiligungsweisen. Die Einzelaktivität AUSPACKEN vollzieht er darüber hinaus per haptischer, die Ensembleaktivität VoRSCHLAGEN per verbaler Beteiligung. Dass die beiden Aktivitäten im Gegensatz zu den strukturell inkompatiblen Aktivitäten, die in Abschnitt 5.1 besprochen wurden und jeweils nur per Abbruch einer der Aktivitäten koordiniert werden konnten, hier zu einem gewissen Grad kompatibel sind, zeigt sich darin, dass der Regisseur die visuelle Beteiligungsweise von der AusPack-Einzelaktivität abziehen kann und trotzdem ein engagement display dafür anzeigen kann (Tab. 9).

Tab. 9: Pausieren der Einzelaktivität bei verbaler Beteiligung an der Ensembleaktivität durch REG.

\begin{tabular}{|c|c|c|c|}
\hline Transkript 6 & a) & b) & c) \\
\hline Einzelaktivität: & engagement display & pausieren & engagement display \\
\hline \multirow{3}{*}{ AUSPACKEN } & - & - & 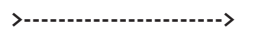 \\
\hline & hap. \& vis. & haptische & haptische \\
\hline & Beteiligungsweisen & Bindung & Beteiligungsweise \\
\hline Ensembleaktivität: & & engagement display & engagement display \\
\hline \multirow[t]{2}{*}{ VORSCHLAGEN } & & 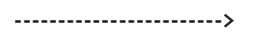 & 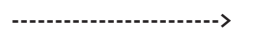 \\
\hline & & $\begin{array}{l}\text { ges., vis. \& verb. } \\
\text { Beteiligungsweisen }\end{array}$ & $\begin{array}{l}\text { vis. \& verb. } \\
\text { Beteiligungsweisen }\end{array}$ \\
\hline
\end{tabular}

\section{Transkript 6: Pausieren der Einzelaktivität AUSPACKEN zur multimodalen Beteiligung an der Ensembleaktivität VoRSCH LAGEN}

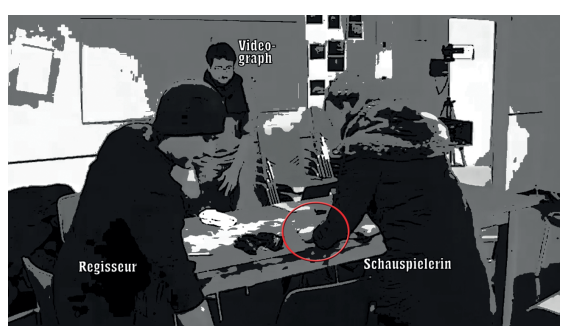

Abb. 6.1: SCW legt das Tablet auf den Tisch.

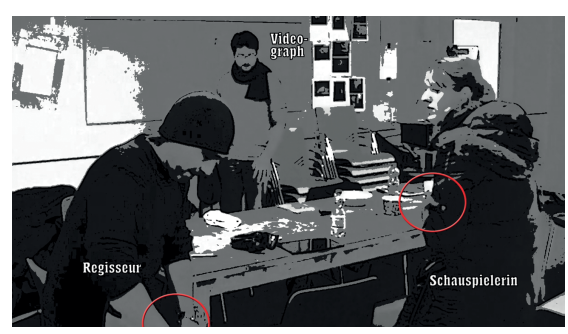

Abb. 6.2: REG packt weiter die Tasche aus.

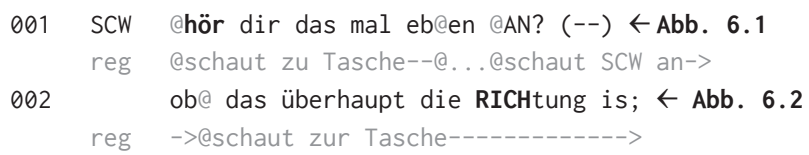




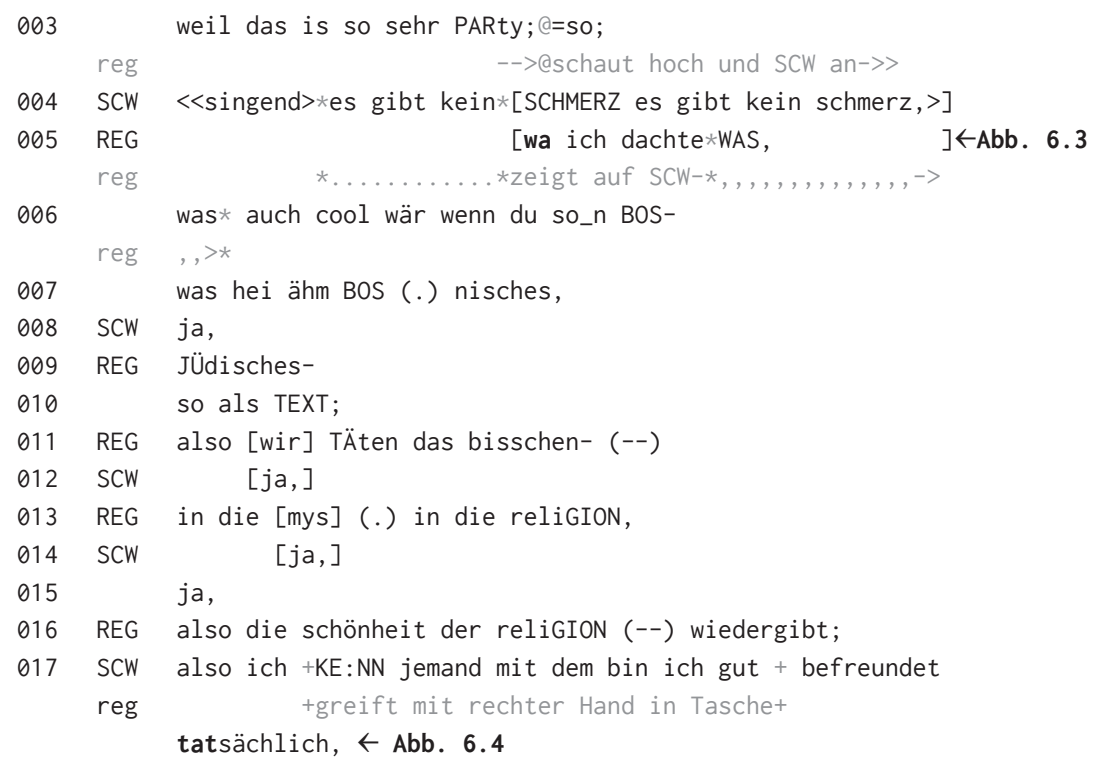

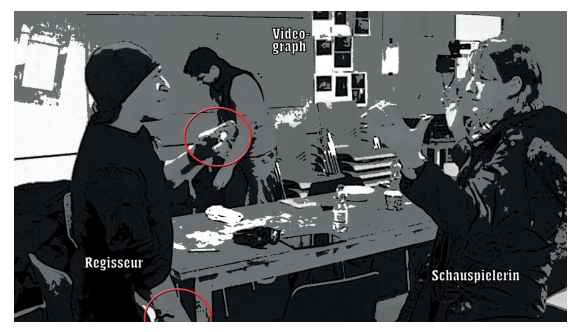

Abb. 6.3: REG zeigt auf SCW.

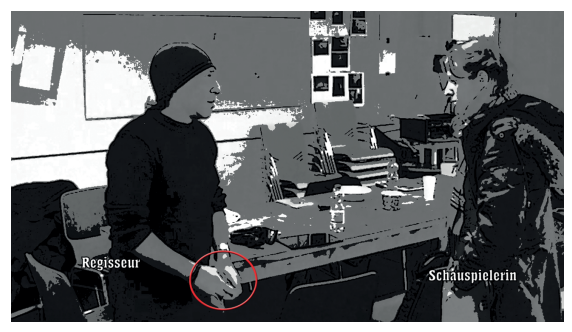

Abb. 6.4: REG holt das Brillenetui aus der Tasche.

a) engagement display bzgl. der Einzelaktivität via haptischer und visueller Beteiligungsweisen (Z.001-002): Während der Regisseur mit seiner rechten Hand die Schnalle seiner Tasche bearbeitet, legt die Schauspielerin das Tablet auf den Tisch (Abb. 6.1). Zeitgleich fordert sie den Regisseur auf, ${ }^{33}$ sich das Lied anzuhören (Z.001-2). Dieser wirft zwar einen kurzen Blick auf das (ausgeschaltete) Tablet, bleibt aber ansonsten weiterhin einzig an seiner Einzelaktivität beteiligt, die er mit beiden Händen bearbeitet und auf die er sich visuell orientiert (Abb. 6.2).

33 In der Terminologie von Couper-Kuhlen (2014: 634) würde die Schauspielerin eine „suggestion“ realisieren, da der Agent und Benefizient der zukünftigen Handlung der Regisseur ist (es ist „sein“ Stück, das durch das Lied bereichert werden kann). 
b) Pausieren der Einzelaktivität durch Aufrechterhalten der haptischen Bindung bei gleichzeitigem engagement display bzgl. der Ensembleaktivität via visueller und verbaler Beteiligungsweisen (Z.003-016): Das engagement display des Regisseurs verändert sich, als die Schauspielerin damit beginnt, ihren Musikvorschlag singend und beidhändig tanzend vorzutragen (Z.004). Der Regisseur produziert auf diese multimodale Gestalt des Vorschlags einen GEGENVORSCHLAG („wa ich dachte WAS/was auch cool wär wenn du so_n BOS“, Z.005-6), den er dergestalt organisiert, dass er mit einer Pseudocleft-Konstruktion (Günthner \& Hopper 2010) auf ihren VoRSCHLAG reagiert, ohne ihn inhaltlich zu bewerten. Gleichzeitig pausiert er zeitgleich mit Einsetzen seiner verbalen Beteiligung an der Ensembleaktivität seine Einzelaktivität. Dazu bewegt er noch während des singend und tanzend vorgetragenen Vorschlags der Schauspielerin seine linke Hand langsam aus der Tasche nach oben und verändert die Form der Hand zeitglich mit dem Start seines überlappenden Redens (Z.005) zu einer auf die Schauspielerin gerichteten Zeigegeste (Abb. 6.3). Mit der pausierten Einzelaktivität kann der Regisseur seine linke Hand zur gestischen Beteiligung an der Ensembleaktivität VoRSCHLAG verwenden, während er mit seiner rechten Hand immer noch in der Tasche verbleibt, den Taschendeckel aufhält und dadurch ein konstantes Display der Involvierung in die Startphase seiner Einzelaktivität AUSPACKEN leistet. Das Pausieren der Einzelaktivität zugunsten der Ensembleaktivität passiert in einem interaktional kritischen Moment, in dem der Regisseur nicht nur verbal und gestisch um den Sprecherstatus (Schegloff 2000b; Mondada \& Oloff 2011) und damit mit der Schauspielerin um die verbale Beteiligung an der Ensembleaktivität konkurriert, sondern auch eine Ablehnung des VorschlaGs und damit eine dispräferierte Handlung projiziert. Das (noch) Nicht-Akzeptieren von VoRschläGEN (Houtkoop-Steenstra 1990) gehört aufgrund der starken Präferenz für Übereinstimmungen in Interaktionen (Sacks 1987) zu den dispräferierten zweiten Paarteilen (Pomerantz 1984; Schegloff 2007). Der Regisseur realisiert im vorliegenden Fall das Nicht-Akzeptieren des Vorschlags der Schauspielerin mithilfe einer indirekten positiven Bewertung „was auch cool wär“ (Z.006). Damit kann er mit einem (GEGEN)VorschlaG als neue, „modifizierte Aktivität“ (Stivers \& Sidnell 2016: 153) ihren VorschlaG ablehnen, ohne ihr „face“ (Goffman 1955; Brown \& Levinson 1987) zu bedrohen. Den pausierten Status seiner Einzelaktivität hält der Regisseur aufrecht, indem er seine rechte Hand den gesamten Turn über an der Taschenlasche hält und damit ein engagement display seiner Einzelaktivität anzeigt. Die etwa eine Sekunde andauernde gestische Preparation, die mit der Pseudocleft-Konstruktion emergiert, ist also zeitgleich der Beginn der Pause der Einzelaktivität, die der Regisseur erst mit dem Ende seiner verbalen Beteiligung an der VorsCHLAG-Ensembleaktivität wieder auflöst (Z.017, Abb. 6.4). Für den Regisseur besteht demnach eine gleichzeitige Relevanz zweier Aktivi- 
täten, die er bearbeitet, indem er eine der Aktivitäten nicht abbricht, sondern pausiert. Dies erreicht er an dieser Stelle dadurch, dass er mit einer Hand die Tasche offenhält (=haptische Beteiligungsweise an der Einzelaktivität TASchE AUSPACKEN und dadurch Relevanthalten der Aktivität), aber keine Gegenstände aus derselben holt (=keine Bearbeitung der Aktivität). Das er an dieser Stelle die Einzelaktivität und nicht die Ensembleaktivität pausierend zurückstellt, ist nicht zufällig, sondern ergibt sich interaktional daraus, dass die Schauspielerin mit ihrem VoRschLAG eine Beteiligung an dieser Aktivität konditionell relevant setzt.

c) duales engagement display bzgl. der gleichzeitig relevanten Aktivitäten durch Aufteilen der Beteiligungsweisen (Z.017): Der Regisseur löst das Pausieren der Einzelaktivität auf, als sein Turn beendet ist und die Schauspielerin die verbale Beteiligung an der Ensembleaktivität wieder aufnimmt (Z.017). Ohne den Blickkontakt mit der Schauspielerin und damit sein engagement display bzgl. der Ensembleaktivität zu verändern, führt er dazu die linke Hand im Rahmen seiner haptischen Beteiligung am AUSPACKEN langsam in die home position (Kendon 1980) zurück, wo sie sich mit der rechten Hand trifft, die ein Brillenetui herausholt (Abb. 6.4).

Damit nutzt der Regisseur in dieser Sequenz seine linke Hand bei der gestischen Herstellung seines Gegenvorschlags, während seine rechte Hand im Rahmen seiner AuSPACK-Aktivität die Lasche seiner Tasche offenhält. Dies ist möglich, weil Vorschlagen und AuSPACKEN unterschiedliche Beteiligungsweisen für ihre Realisierung benötigen. Auffällig ist, dass der Regisseur seine Einzelaktivität pausiert, noch bevor er mit der Schauspielerin überlappend um die Ensembleaktivität bzw. den Status des Rederechts konkurriert (Z.003) und sie erst wieder fortsetzt, nachdem sein GEGENVORSCHLAG abgeschlossen ist und die Schauspielerin ihm ein ANGEBOT unterbreitet (Z.017). Das legt einerseits einen gewissen Grad an Vorbereitung nahe, die der Regisseur aufwenden muss, um seinen für die Schauspielerin potentiell gesichtsbedrohenden GEGENVORSCHLAG zu realisieren. Andererseits zeigt es, dass die Einzelaktivität nicht zufällig, sondern in Relation zur Ensembleaktivität an einer interaktional funktionalen Stelle pausiert und fortgesetzt wird. Das Pausieren der Einzelaktivität erfolgt hier, um die multimodale Beteiligung an der Ensembleaktivität möglich zu machen. Nach Abschluss des Turns als verbale Beteiligungsweise wird die pausierte Aktivität fortgesetzt, sodass der Regisseur kurzzeitig simultan ${ }^{34}$ in beide Aktivitäten involviert ist (per haptischer Beteiligungsweise an der Einzelaktivität und per visueller Beteiligungsweise via Blickkontakt an der Ensembleaktivität). Obwohl das

34 Solche simultanen Koordinierungen werden in 5.3 eingehend beschrieben. 
Pausieren der Einzelaktivität einen gewissen Grad an Vorbereitung bei der Einbettung in den interaktionalen Verlauf deutlich werden lässt, hat die pausierte Einzelaktivität lediglich (intrapersonell-koordinativen) Einfluss auf den pausierenden Regisseur, nicht aber in Bezug auf die interpersonelle Organisation mit der Ko-Interagierenden.

Diese Beobachtung verhält sich diametral zur interaktionalen Organisation im folgenden Ausschnitt. Dort erfolgt das Pausieren der Einzelaktivität RUCKSACK ABSETZEN nicht im Rahmen einer verbalen Beteiligung an einer Ensembleaktivität, sondern nach einer Adressierung. Im Transkriptausschnitt sind Schauspieler (SCM) und Hospitant (HOM) in eine BEGRÜßUNG involviert, während parallel dazu Schauspielerin (SCW), Hospitantin (HOW), Regieassistentin (ASS) und Videograph (VID) einen Vorschlag der Hospitantin erörtern, das gemeinsame Kind der Figuren an einer Operation sterben zu lassen.

Tab. 10: Pausieren der Einzelaktivität bei Beteiligung an der Ensembleaktivität durch SCM.

\begin{tabular}{|c|c|c|c|c|}
\hline Transkript 7 & a) & b) & c) & d) \\
\hline Einzelaktivität: & & starten & pausieren & engag. display \\
\hline RUCKSACK & & 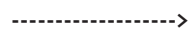 & 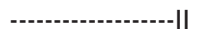 & 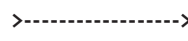 \\
\hline \multirow[t]{2}{*}{ ABSETZEN } & & haptische & haptische & hapt. \& vis. \\
\hline & & Beteiligungsw. & Bindung & Beteiligungsw. \\
\hline \multirow{5}{*}{$\begin{array}{l}\text { Ensembleaktivität: } \\
\text { VoRSCHLAGEN }\end{array}$} & disengag. & engag. display & engag. display & \\
\hline & display & & & \\
\hline & (n) & - & - & \\
\hline & & visuelle & vis. \& verb. & \\
\hline & & Beteiligungsw. & Beteiligungsw. & \\
\hline
\end{tabular}

Der Schauspieler wird per Adressierung in diese Ensembleaktivität inkludiert, wobei er seine Einzelaktivität RuCKSACK ABSETZEN pausiert. Im Zuge der Pausierung beteiligt sich der Schauspieler visuell und verbal an der Ensembleaktivität (Tab. 10). Anders als im vorherigen Transkript 6 erfolgt das Pausieren der Einzelaktivität hier nicht zum primären Zweck einer intrapersonellen Koordinierung der (verbalen) Beteiligung, sondern im Rahmen einer interpersonellen Koordinierung bei inkludierender Adressierung an den Schauspieler. Diese Inkludierung in die Ensembleaktivität durch die Hospitantin lädt den Schauspieler in den gemeinsamen Interaktionsraum ein (Besch 1996; Droste \& Günthner 2020; Hartung 2001). Dies erfolgt dabei zu einem Zeitpunkt, an dem der Schauspieler gerade eine durch ein schisming (Egbert 1993, 1997) ausgelöste Trennung der Interaktion in zwei parallele Interaktionsstränge aufgelöst hat und kurzzeitig kein Beteiligter einer Interaktion ist. Pausiert wird dabei nicht mit der Adressierung, sondern erst nach einer epistemischen Relevantsetzung der Beteiligung des 
Schauspielers. Dies ist keineswegs verwunderlich, da die pronominale Adressierung in Mehrpersonen-Interaktionen zunächst immer ambig ist und weiterer Kontextualisierungshinweise zum Verständnis bedarf (vgl. Lerner 1996, 2003).

\section{Transkript 7: Pausieren der Einzelaktivität RUCKSACK ABSETZEN bei Inkludierung in Ensembleaktivität VORSCH LAGEN ${ }^{35}$}

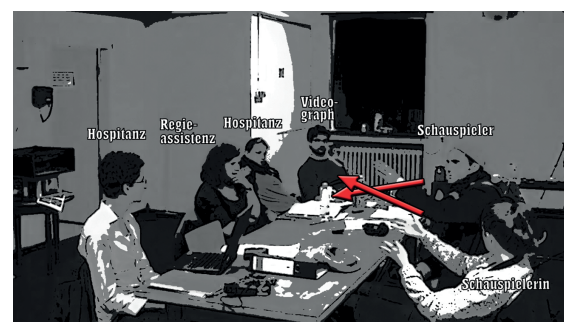

Abb. 7.1: Beteiligungsweise „über Kreuz“.

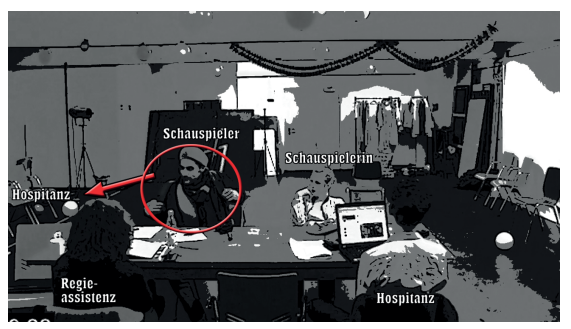

Abb. 7.2: SCM pausiert das Absetzen des Rucksacks.

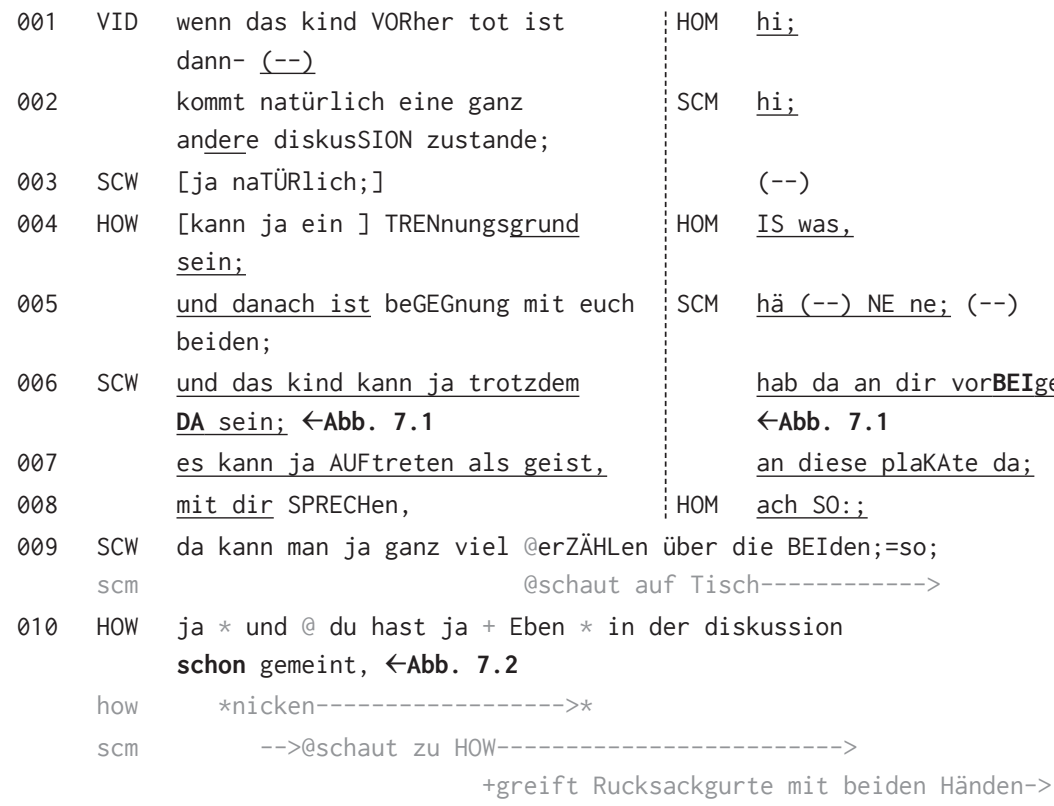

+greift Rucksackgurte mit beiden Händen->

35 Die Transkription des schisming orientiert sich an Egbert (1993). Ergänzend liefern unterstrichene Formatierungen Informationen über die simultanen Sprachbeiträge. 
011

$\mathrm{scm}$

012

$013 \mathrm{SCW}$

$014 \mathrm{HOW}$

015 SCM

$\mathrm{scm}$

016

017

018

019

020

021

022

023 +es kann das ende einer Ehe sein;

+hält Hände an Rucksackgurten-->

wenn das kind TOT ist; =glaub ich;

SCHAFfen sie_s (.) schaffen sie_s nich;

oder der TOD ist genau was euch wieder zusammenschweißt;

hoo० @ (---)

$-->$ @schaut auf Tisch->

da sind wir voRher auseinander; $\leftarrow$ Abb. 7.3

[sozu]sagen;=oder was? (--)

SCW [hmhm]

SCM die @diskussion hat uns entZWEIT,

scm -->@schaut SCW an-------------->

und der TOD bringt uns wieder zusammen?

SCW hmhm,

HOW vielLEICHT;=ja;

SCM @((lacht))

scm @schaut auf Tisch-->>

SCW geht (.) +geht WIRKlich; $\leftarrow$ Abb. 7.4

scm -->+setzt Rucksack ab--->>

SCW ich hab auch mal ein STÜCK gesehen;

da ging_s total um_n KIND;

das KIND ist geSTORben;

das erste KIND?

und es wurd kein einziges mal erWÄHNT auf der bühne;

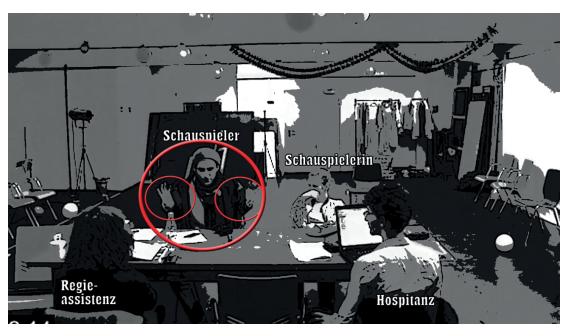

Abb. 7.3: SCM gestikuliert mit dem Rucksack.

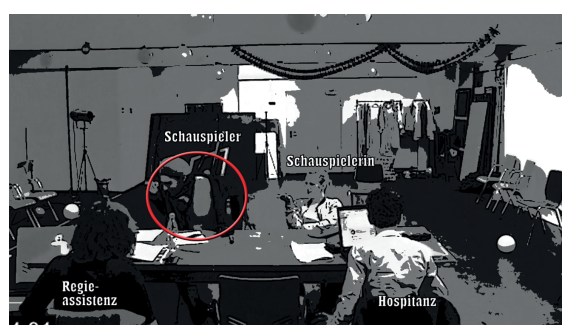

Abb. 7.4: SCM setzt den Rucksack ab.

a) disengagement display nach Auflösen eines schismings (Z.001-009): Zu Beginn des Transkriptausschnitts (Z.001-8) sind zwei Ensembleaktivitäten innerhalb eines Interaktionsensembles beobachtbar, die parallel zueinander verlaufen. Dieses Phänomen, das Egbert (1993) als schisming konzeptualisiert, besteht hier zum einen aus der Bearbeitung des VorschlaGs durch Videograph, Hospitantin, Schauspielerin und Regieassistentin, die in dieser Sequenz zwar verbal abstinent 
bleibt, aber ebenfalls ein visuelles engagement display bezüglich dieser Ensembleaktivität produziert. Zum anderen sind Schauspieler und Hospitant Beteiligte einer dyadischen Ensembleaktivität BEGRÜßUNG, in der sie aushandeln, dass der Schauspieler Plakate hinter dem Hospitanten fixiert und nicht den Hospitanten selber angeschaut hat. Die Parallelität der zwei Ensembleaktivitäten führt hier dazu, dass die Beteiligungsweisen an den jeweiligen Aktivitäten einander verbal überlappen (Z.001-8) und körperlich (gestisch bzw. proxemisch) überkreuzen (Abb. 7.1). Die Gleichzeitigkeit der parallelen Ensembleaktivitäten löst sich auf, nachdem Hospitant und Schauspieler ihre Aktivität zu einem Abschluss bringen (Z.008) und der Hospitant sich wieder seinem Laptop und damit seiner Einzelaktivität IM NEwsFEED LESEN zuwendet, während der Schauspieler seinen Blick auf den Tisch richtet (Z.009). Auch wenn die Möglichkeit besteht, dass der Schauspieler den Verlauf der Ensembleaktivität VoRSCHLAGEN auditiv monitort, zeigt er weder Involvierung in die soeben aufgelöste Ensembleaktivität BEGRÜßUNG, noch bzgl. der fortdauernden Vorschlags-Ensembleaktivität an. Er ist somit für kurze Zeit zwar Mitglied einer sozialen Situation (Goffman 1963), aber kein Mitglied einer Aktivität. ${ }^{36}$

b) duales engagement display bzgl. beider ko-relevanter Aktivitäten (Z.009-010): Dieses disengagement display hält der Schauspieler aufrecht, bis er von der Hospitantin pronominell und mithilfe einer nickenden Kopfgeste (Goodwin 1980b; Stivers 2010) adressiert und damit in die Ensembleaktivität inkludiert wird (,ja und du hast ja Eben in der diskussion schon gemeint,“, Z.010). Erst mit der Adressierung richtet sich der Schauspieler auf und schaut die Hospitantin an, was nach Egbert (1993:271) ein typisches Verfahren ist, mit der sich Teilnehmende wieder in auseinandergebrochene Interaktionen eingliedern. Den Blickkontakt mit der Hospitantin aufrechterhaltend fasst der Schauspieler innerhalb eines rhythmischen Schlags ${ }^{37}$ nach der Adressierung mit beiden Händen die Gurte seines Rucksacks (Z.010), an denen er zwei weitere rhythmische Schläge später zieht (Abb. 7.2). Damit zeigt er sowohl via visueller Beteiligungsweise ein engagement display bzgl. der Ensembleaktivität an als auch Involvierung in die Startphase seiner Einzelaktivität per haptischer Beteiligungsweise.

36 Mit Goffman (1963:69-73) gesprochen ist der Teilnehmendenstatus des Schauspielers hier der eines self-involvements: „While outwardly participating in an activity within a social situation, an individual can allow his attention to turn from what he and everyone else considers the real or serious world, and give himself up for a time to a playlike world in which he alone participates. This kind of inward emigration from the gathering may be called ,away [...].“

37 Auer \& Couper-Kuhlen (1994: 85-86) definieren Schläge folgendermaßen: „Sequenzen von Akzenten [werden] nach bestimmten Prinzipien zu rhythmischen Gestalten zusammengefaßt. Wir nennen Akzente, die in solchen Gestalten eine Rolle spielen, Schläge.“ 
c) Pausieren der Einzelaktivität durch Aufrechterhalten der haptischen Bindung bei gleichzeitigem engagement display bzgl. der Ensembleaktivität via visueller und verbaler Beteiligungsweisen (Z.011-018): Anstatt jedoch in die Kernphase der Einzelaktivität überzugehen, pausiert der Schauspieler das ABSETZEN SEINES RucKSACKS, während er mithilfe seiner Körperorientierung und dem Blickkontakt weiterhin Involvierung in die Ensembleaktivität anzeigt. Das Aktivitätspausieren erfolgt in jenem Moment, in dem die Hospitantin mit dem Hinweis „schon gemeint“ (Z.010) auf eine vorherige Beteiligung des Schauspielers an der VoRscHLAGS-Ensembleaktivität referiert und diese nun epistemisch relevant setzt. Der Schauspieler trägt dem Rechnung, indem er die Einzelaktivität pausiert und erst dann wieder fortsetzt, als er diese epistemische Relevanz als aufgehoben behandelt (Z.024). Bis zu diesem Zeitpunkt hält er die Einzelaktivität pausiert und durchgängig relevant, indem er seine Hände an den Rucksackgurten lässt und sich in dieser körperlichen Konfiguration neben verbalen Beiträgen (Z.015-23) auch gestisch an der Ensembleaktivität beteiligt (Abb. 7.3). Die pausierte Einzelaktivität bestimmt damit die Gestalt, mit der er sich gestikulierend an der Ensembleaktivität beteiligen kann.

d) Fortsetzen der pausierten Einzelaktivität nach Abschluss der Ensembleaktivität (Z.019-030): Erst als der Schauspieler den gemeinsam erarbeiteten VoRSCHLAG zusammenfasst (,die diskussion hat uns entZWEIT / und der TOD bringt uns wieder zusammen?", Z.019-20) und darauf sowohl von der Schauspielerin (Z.021) als auch der Hospitantin (Z.022) Bestätigungen erhält, setzt er seine pausierte Einzelaktivität fort (Z.024, Abb. 7.4). Dazu zieht er seine visuelle Beteiligungsweise von der Ensembleaktivität ab und reorientiert sich auf den Rucksack, den er auf im Rahmen seiner haptischen Beteiligungsweise auf den Boden stellt. Dies ist ebenfalls der Moment, den auch die Schauspielerin als Abschluss des Vorschlags interpretiert, indem sie eine neue Ensembleaktivität initiiert, in der sie von einem ähnlichen Fall in einem anderen Theaterstück BERICHTET (Z.024-30). Nach der Pausierung der Einzelaktivität organisiert der Schauspieler seine Beteiligung hier, wie bereits der Regisseur im vorherigen Transkript 6, dergestalt, dass er trotz pausierter Einzelaktivität gestikulierend zur Ensembleaktivität beitragen kann (Abb. 7.3). Anders als der Regisseur, der eine Hand zum Pausieren und die andere zum Gestikulieren verwendet, macht der Schauspieler beim Gestikulieren von beiden Händen Gebrauch, während sie die Einzelaktivität in der Startphase pausiert halten. Das Aufrechterhalten der Relevanz der pausierten Einzelaktivität wird dadurch in die Gestalt seiner multimodalen Beteiligung an der Ensembleaktivität integriert und erst wieder aufgelöst, als die Ensembleaktivität von den Teilnehmenden als abgeschlossen behandelt wird (Tab. 10). 
Sowohl in Transkript 6 als auch in Transkript 7 organisieren die Pausierenden ihre multimodalen Gestalten so, dass sie mithilfe von Blickkontakt, Körperorientierung, Gestik und verbalen Beiträgen durchgängig engagement displays bzgl. der Ensembleaktivität anzeigen können. Darin unterscheidet sich das folgende Transkript 8, in dem im Unterschied zu Transkript 6 und Transkript 7 die Ensembleaktivität zugunsten einer Einzelaktivität pausiert wird. In diesem Ausschnitt formuliert die Schauspielerin einen VorschlaG bzgl. der Inszenierung, während sie ihren MANTEL AUSZIEHT. Bei diesen beiden Aktivitäten werden keine Beteiligungsweisen doppelt benötigt, weshalb die Aktivitäten zunächst strukturell kompatibel sind. Dass es in der folgenden Sequenz an zwei Stellen zum Pausieren der Ensembleaktivität kommt, hat damit zu tun, dass Probleme beim Vollzug der Einzelaktivität auftreten: Zuerst muss ein Ablageort lokalisiert werden und danach verfängt sich der Arm im Mantelärmel. Beide Probleme bedürfen zum Bearbeiten eine visuellen Beteiligungsweise seitens der Schauspielerin. Das Pausieren der Ensembleaktivität realisiert die Schauspielerin bei beiden Stellen, indem sie die visuelle Beteiligungsweise von dieser Aktivität abzieht und auch die verbale Beteiligung einstellt, aber in ihrer syntaktischen Projektion bzw. epistemischen Rahmung eine Fortsetzung des verbalen Beitrags und damit der Ensembleaktivität erwartbar macht.

Tab. 11: Pausieren der der Ensembleaktivität bei Realisierung der Einzelaktivität durch SCW in Z.002 \& 007.

\begin{tabular}{|c|c|c|c|}
\hline \multicolumn{4}{|l|}{ Transkript 8} \\
\hline Einzelaktivität: & engagement display & engagement display & engagement display \\
\hline \multirow[t]{3}{*}{ AusZIEHEN } & - & - & - \\
\hline & haptische & haptische $\&$ visuelle & haptische \\
\hline & Beteiligungsweise & Beteiligungsweisen & Beteiligungsweise \\
\hline Ensembleaktivität: & engagement display & pausieren & engagement display \\
\hline \multirow[t]{3}{*}{ VORSCHLAGEN } & - & - & >-'- \\
\hline & visuelle \& verbale & epistem. Rahmung / & visuelle \& verbale \\
\hline & Beteiligungsweisen & syntak. Projektion & Beteiligungsweisen \\
\hline
\end{tabular}

Ebenfalls Teil des Interaktionsensembles sind die Hospitantin, die ihren TEE ZUBEREITET, der Hospitant, der an seinem Laptop GoogELT, der Videograph, der an seinem Laptop arbeitet und dort DATEIEN KONVERTIERT sowie die Regieassistentin, die als einzige in keine Einzelaktivität involviert ist. Als Konsequenz aus diesen Involvierungen ergibt sich für die Ensembleaktivität ein eingeschränkter Teilnehmendenkreis, bei dem lediglich die Regieassistentin durchgehend ein engagement display bzgl. der Ensembleaktivität anzeigt. Während Hospitant und 
Hospitantin keine solche Involvierung anzeigen, wechselt der Videograph zwischen seiner visuellen Orientierung auf den Laptop und die Schauspielerin. Er macht damit deutlich, dass seine Laptop-Einzelaktivität und die Ensembleaktivität strukturell bzgl. des engagement displays hinsichtlich der visuellen Beteiligungsweise inkompatibel sind. Auffällig ist dabei, dass diese Orientierungswechsel in solchen Momenten geschehen, in denen die Schauspielerin verbale Pausen realisiert und damit die Ensembleaktivität kurzzeitig pausiert.

\section{Transkript 8: Pausieren der verbalen Beteiligung an der Ensembleaktivität VoRSCHLAGEN beim Aufrechterhalten der Einzelaktivität MANTEL AUSZIEHEN}

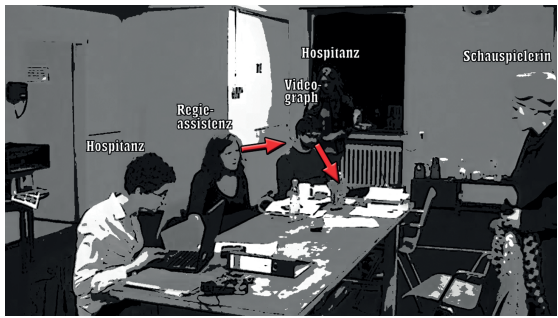

Abb. 8.1: SCW zieht den Reißverschluss herunter.

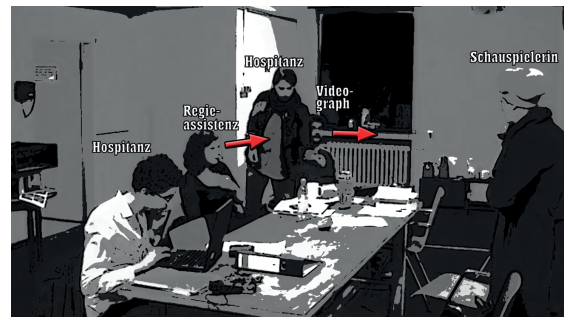

Abb. 8.2: SCWs linke Hand verfängt sich im Mantelärmel.

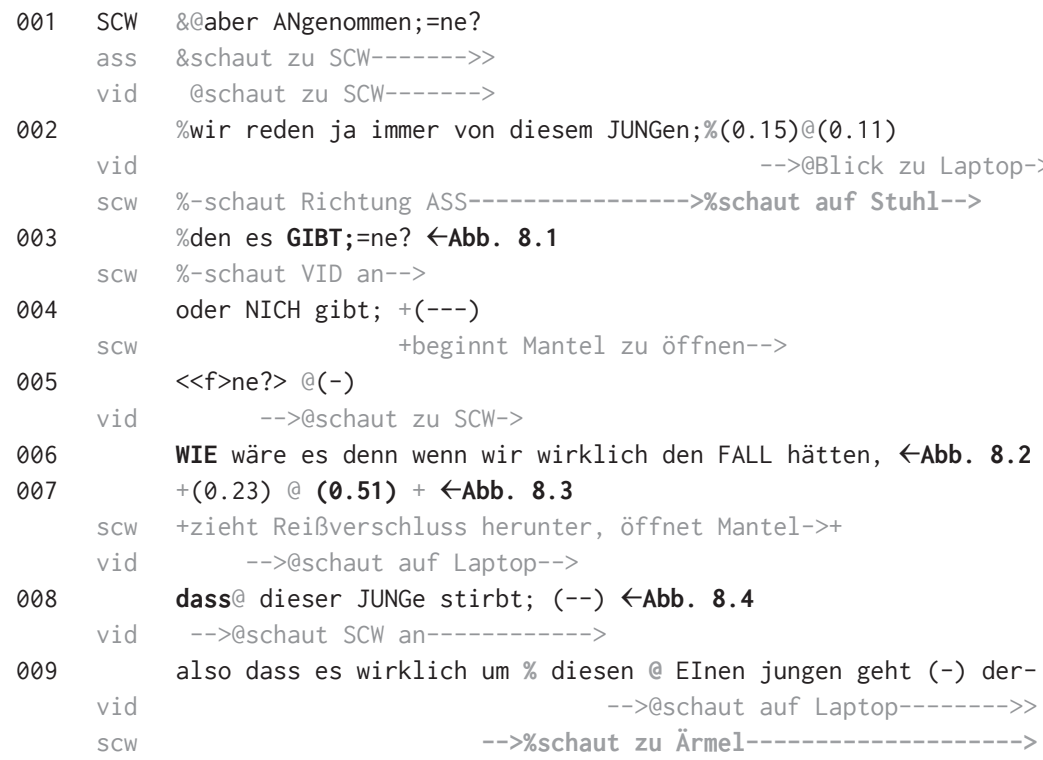


010

SCW

011 SCW

013 SCW \%der EHRgeiz der eltern bringt das kind um. (--)

\%schaut Richtung ASS--

und das [gibt_s dann am ende zu] verAN-

[ja: : :-

=also man erzählt so RÜCKblickend;=eher;

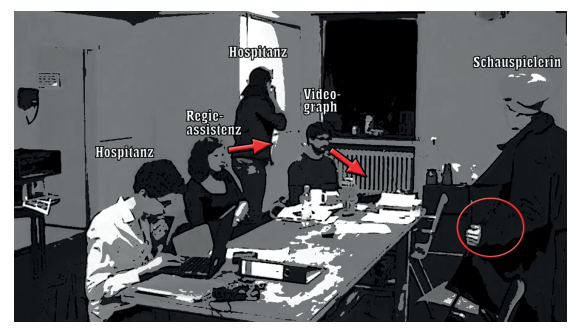

Abb. 8.3: VID löst den Blickkontakt mit SCW auf.

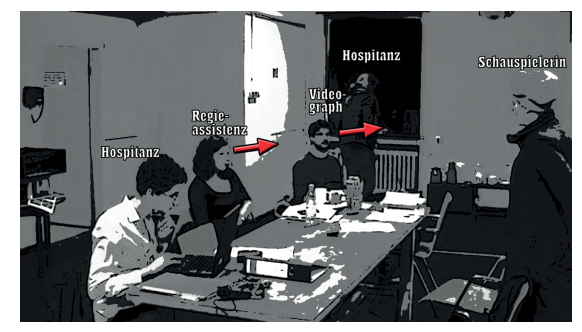

Abb. 8.4: VID schaut zu SCW.

a) Erstes Pausieren der Ensembleaktivität durch Abzug der verbalen und visuellen Beteiligungsweisen bei gleichzeitiger Fortsetzungsprojektion via epistemischer Rahmung (Z.001-003): Während die Schauspielerin sich zu ihrem Platz am Tisch bewegt und ihren Rucksack abstellt, verweist sie auf den common ground (Clark 1996, 2006; Clark, Schreuder \& Buttrick 1983) der Beteiligten und stellt damit den epistemischen Rahmen ihres VorschLAGS her (Z.001-3). Im Verlauf dessen erhält sie von der Regieassistentin und dem Videographen, die sie beide anschauen, engagement displays (Z.001). Während die Regieassistentin dieses display die gesamte Sequenz über beibehält, wendet der Videograph sich wieder seiner Einzelaktivität zu, als die Schauspielerin eine kurze verbale Pause einlegt (Z.002). Durch die epistemische Rahmung macht sie an dieser Stelle einen Turnabschluss unwahrscheinlich und eine Fortsetzung ihres Interaktionsbeitrags erwartbar. Die Regieassistentin, die neben der Schauspielerin zu diesem Zeitpunkt als einzige Beteiligte Involvierung anzeigt (Abb. 8.1), trägt dem Rechnung, indem sie den Turn der Schauspielerin trotz der Abschlussmarkierung in Form der tag question (Heritage \& Raymond 2005) „ne“ (Z.003) als nicht abgeschlossen behandelt und bis auf das engagement display keine weiteren Beteiligungsweisen (wie Nicken oder verbale Beteiligung) realisiert. Während also der Videograph die verbale Pause als Slot interpretiert, in dem er sich von der Ensembleaktivität zurückziehen und sich seiner Einzelaktivität zuwenden kann, verbleibt die Regieassistentin in ihrem visuellen engagement display. Dies behält sie auch dann bei, als die Schauspielerin nach Ausbleiben einer Reaktion der Ko-Interagierenden ihre verbale Beteiligung zunächst inkrementell erweitert, dann abermals pausiert (Z.004) und im Rahmen ihrer Einzelaktivität MANTEL AUSZIEHEN ihren Mantel zu öffnen beginnt. 
b) Zweites Pausieren der Ensembleaktivität durch Abzug der verbalen und visuellen Beteiligungsweisen bei gleichzeitiger Fortsetzungsprojektion via syntaktischer Projektion (Z.004-009): Die Pause der verbalen Beteiligung an der Ensembleaktivität mit dem Start der Einzelaktivität würde den anderen Interagierenden die Möglichkeit eröffnen, an dieser Stelle den common ground zu ratifizieren. Als dies abermals nicht geschieht, produziert die Schauspielerin einen prosodisch auffälligen (erhöhte Lautstärke, stark steigende Intonation) Diskursmarker „ne?“ (Z.005), die die Aufmerksamkeit der Anwesenden einfordert (vgl. Hagemann 2009; König 2017). Im Unterschied zum ersten ne evoziert die Schauspielerin hier die Aufmerksamkeit des Videographen. Dieser pausiert seine Einzelaktivität und schaut sie an (Z.005, Abb. 8.2). Mit zwei auf sie orientierten Beteiligten führt die Schauspielerin nun ihre Idee aus, der Junge könnte sterben (Z.006), während sie weiterhin im Zuge ihrer Einzelaktivität ihren Mantel öffnet. Als die Schauspielerin daraufhin abermals ihre verbale Beteiligung pausiert (Z.007), hält der Videograph zunächst für ca. 0.2Sekunden den Blickkontakt, bevor er seinen Blick wieder auf seinen Laptop richtet und damit seine Involvierungsanzeige bzgl. der Ensembleaktivität auflöst (Abb. 8.3). Dass seine Beteiligung an der Ensembleaktivität zumindest hier eng mit der verbalen Beteiligung der Schauspielerin zusammenhängt, wird deutlich, als der Videograph seinen Blick der Schauspielerin zuwendet, sobald diese ihre verbale Beteiligung wieder aufnimmt (Z.008, Abb. 8.4). Dieses engagement display hält der Videograph aufrecht, bis die Schauspielerin ihren Vorschlag zu einem möglichen Abschluss bringt und ihn im Folgenden als dyadische Ensembleaktivität mit der Regieassistentin weiterbearbeitet. Das prosodische wie auch das syntaktische Format der Äußerung „WIE wäre es denn wenn wir wirklich den FALL hätten,“ (Z.006) macht an dieser Stelle für die Teilnehmenden erwartbar, dass die Schauspielerin ihren Turn und damit ihre verbale Beteiligung an der Ensembleaktivität nach der auf diese TCU folgenden Pause, fortsetzt. Sowohl die leicht steigende prosodische Kontur der Äußerung als auch der unvollständige Konditionalsatz projizieren die zeitnahe Fortsetzung (bzw. den Abschluss) des Beitrags. Solange dies nicht eingelöst wird, kann die Aktivität als pausiert gelten. Diese Interpretation der pausierten Ensembleaktivität wird von der Regieassistentin geteilt, indem sie die Ensembleaktivität nicht als abgebrochen behandelt, sondern via visueller Beteiligungsweise weiterhin ein engagement display bzgl. dieser Aktivität produziert. Im Gegensatz dazu nutzt der Videograph die Pause, um seine eigene pausierte Einzelaktivität wieder zu bearbeiten.

Damit kommt es in dieser Sequenz an zwei Stellen zu Veränderungen in der Teilnehmendenrahmung, die mit verbalen Beteiligungspausierungen in Zusammenhang gebracht werden können: Z.002 und Z.007. Bei erstgenannter Stelle bricht 
der Videograph sein visuelles engagement display nach 0.15 Sekunden ab, das er erst wieder aufnimmt, als die Schauspielerin auf eine tag question zurückgreift. Auffällig ist hier, dass der Videograph seinen Blick erst senkt, als die Schauspielerin dies auch tut. Umso interessanter ist die zweite Stelle (Z.007) im direkten Vergleich dazu. Denn auch hier zieht sich der Videograph nach 0.23 Sekunden zurück, als die verbale Beteiligung der Sprecherin ausbleibt und die Schauspielerin stattdessen ihre Einzelaktivität bearbeitet. Anders als in der Zeile 002 erhält er hier aber durchgängig den Blick der Sprecherin. Dass seine Involvierungsorientierung an dieser Stelle eng mit dem verbalen Pausieren der Beteiligung der Schauspielerin an der Ensembleaktivität zusammenhängt, wird deutlich, als er seine visuelle Involvierungsanzeige in genau dem Moment wieder verändert, in dem die Schauspielerin ihren Turn fortsetzt. Damit hat sowohl das Pausieren als auch das Fortsetzen der Ensembleaktivität Einfluss darauf, wie und wann der Videograph seine Involvierung organisiert. Diese durch multiple Aktivitäten geprägte Beteiligungsweise des Videographen unterscheidet sich stark von der monoaktiven Beteiligung der Regieassistentin, die die Rederecht innehabende Schauspielerin durchgängig anschaut (vgl. Goodwin 1979, 1980a; Goodwin 1980b; Goodwin 1984; Lerner 2003). Damit wird deutlich, welche Bedeutung die Pausierungen der Ensembleaktivität für die interaktionale Struktur der sozialen Situation haben: Sie ermöglichen den Teilnehmenden Slots, in denen sie Einzelaktivitäten bearbeiten können (z. B. am Computer arbeiten, einen Mantelablageort finden oder einen Ärmel aus einem Mantel befreien), ohne dass die Relevanz der Ensembleaktivität dadurch aufgehoben werden würde. Wie Hoey (2015, 2018b) anhand von Videodaten zeigt, sind verbale Pausen keinesfalls auf Nicht-Sprechen reduzierbar, sondern tragen Anteil an der intersubjektiven Organisation multimodaler Interaktionen. Darüber hinaus zeigt die Analyse von Transkript 8, dass verbale Pausen als Verfahren zur Pausierung einer Ensembleaktivitä ${ }^{38}$ in enger Relation zur Koordinierung von Einzelaktivitäten stehen. Pausierungen bzw. Fortsetzungen pausierter Ensembleaktivitäten haben Auswirkungen auf die Teilnehmendenrahmung und liefern einen Hinweis darauf, dass an diesen Stellen eine kurzzeitige Veränderung im Relevanzsystem der multiplen Aktivitäten durch die Interagierenden bearbeitet wird. Das Ausbleiben der verbalen Beteiligung an der Ensembleaktivität zieht hier eine Veränderung im participation framework nach sich, die dadurch mitkonstituiert wird, dass die Schauspielerin die Pausierung nicht explizit als solche mar-

38 An dieser Stelle könnte der Eindruck entstehen, Ensembleaktivitäten würden primär durch die verbale Beteiligung konstituiert. Obwohl dies auf viele der in diesem Kapitel besprochenen Aktivitäten (z. B. VoRSCHLAGEN oder BERICHTEN) zutreffen mag, werden später in diesem Kapitel mit Aushändigen DER AKTUELlen StÜCKFASSUng und mit TANZEN in Kapitel 6 ebenfalls zwei sprachfreie Ensembleaktivitäten vorgestellt. 
kiert. Bei Keisanen, Rauniomaa \& Haddington (2014) werden solche Pausierungen mit „wait“ oder „hang on“ als pausiert gekennzeichnet, was es den Ko-Teilnehmenden ermöglicht, sich auf den pausierten Zustand der gemeinsamen Aktivität einzustellen und die Pausierungen mitherzustellen. In ihrem 36-stüdigen Korpus konnten Keisanen, Rauniomaa \& Haddington (2014) lediglich 30 Vorkommen von (Ensemble-)Aktivitätspausierungen in Situationen mit multiplen Aktivitäten beobachten, was einen Eindruck davon vermittelt, wie selten Ensembleaktivitäten zugunsten von Einzelaktivitäten pausiert werden. Dies lässt sich damit erklären, dass Einzelaktivitäten deutlich einfacher zu pausieren sind als Ensembleaktivitäten, da pausierte Beteiligungsweisen an Ensembleaktivitäten für andere Beteiligte von Aktivitätsabbrüchen (siehe 5.1) kaum unterscheidbar sind, wenn keine Involvierungsmarker verwendet werden (siehe 5.3). Pausierte Einzelaktivitäten ermöglichen den Teilnehmenden eine Bandbreite an multimodalen Beteiligungsweisen an den Ensembleaktivitäten (Transkripte 6 \& 7), während pausierte Ensembleaktivitäten den Interaktionsfluss zum Erliegen bringen und sich auf die Teilnehmendenrahmung auswirken können (Transkript 8).

Tab. 12: Schema einer Aktivitätspausierung bei multiplen Aktivitäten.

\begin{tabular}{|c|c|c|c|}
\hline \multirow[t]{2}{*}{ Aktivität 1} & engagement display & $\begin{array}{l}\text { Pausieren durch } \\
\text { Fortsetzungsprojektion }\end{array}$ & engagement display \\
\hline & $\begin{array}{l}\text { z. B. haptische } \\
\text { Beteiligungsweise }\end{array}$ & z. B. haptische Bindung & $\begin{array}{l}\text { z. B. haptische } \\
\text { Beteiligungsweise }\end{array}$ \\
\hline \multirow[t]{2}{*}{ Aktivität 2} & engagement display & engagement display & engagement display \\
\hline & z. B. visuelle \& & z. B. visuelle \& verbale & z. B. visuelle \& \\
\hline
\end{tabular}

Die Aktivitätspausierungen in den Transkripten 6-8 haben gemein, dass die Interagierenden (im Unterschied zu Aktivitätsabbrüchen in 5.1) durchgängig engagement displays bezüglich der konkurrierenden Aktivität aufrechterhalten, während sie die pausierte Aktivität währenddessen simultan relevant halten. Das Relevanthalten wird erreicht, indem die Teilnehmenden eine konstante Fortsetzungsprojektion leisten, die sich für Einzel- und Ensembleaktivitäten unterscheidet. Zum Pausieren von Einzelaktivitäten werden zentrale Handgriffe der Aktivität angehalten, während mindestens ein Element darauf hinweist, dass die Aktivität (jederzeit) wiederaufgenommen werden kann (z. B. indem die Lasche der Tasche mit einer Hand offengehalten wird oder indem der Rucksack nicht abgesetzt wird, während weiterhin die Hände die Riemen umfasst halten). Da Ensembleaktivitäten als eher interpersonell koordinierte und intersubjektiv ausgehandelte 
Einheiten im Gegensatz zu den vorrangig intrapersonell koordinierten Einzelaktivitäten immer auf die reflexive Mitarbeit der Teilnehmenden angewiesen sind, haben Pausierungen von Ensembleaktivitäten zugunsten von Einzelaktivitäten mehr oder weniger starke Auswirkungen auf die Teilnehmendenkonstellation. In Transkript 8 pausiert die Schauspielerin die Ensembleaktivität, indem sie die verbale Beteiligung kurzzeitig anhält. Dass es sich hier um keine Abbrüche, sondern um Pausierungen handelt, die eine zeitnahe Fortsetzung der verbalen Beteiligung projizieren, wird mit dem Blick auf das Turndesign hinsichtlich prosodischer und/oder syntaktischer Formate deutlich (Transkript 8). Die Pausierung einer multiplen Aktivität wird in situ durch die Fortsetzungsprojektion als solche accountable gemacht und in der Projektion ihrer zeitnahen Fortsetzung der Aktivität erkennbar.

\subsubsection{Fortsetzung einer pausierten Aktivität bei simultaner Beteiligung an anderer Aktivität}

Um pausierte Aktivitäten fortzusetzen, werden von den Teilnehmenden diejenigen Beteiligungsweisen reaktiviert, die für das Aufrechterhalten der Fortsetzungsprojektion verwendet werden. Diese Reaktivierungen erfolgen in den folgenden drei Fällen an interaktional relevanten Stellen, wenn Interagierende den aufrechterhaltenen Aktivitäten einen abgeschlossenen Zustand zuschreiben, z. B. wenn eine Ensembleaktivität abgebrochen (Transkript 9), die konditionelle Relevanz eingelöst (Transkript 10) oder eine Handlungssequenz gemeinsam zu Ende gebracht wurde (Transkript 11).

Im ersten der an dieser Stelle diskutierten Fortsetzungsfälle pausiert der Schauspieler seine Einzelaktivität JACKE ABLEGEN, die er primär per haptischer Beteiligungsweise vollzieht, während er per visueller und verbaler Beteiligungsweise an einer Ensembleaktivität partizipiert. Diese beinhaltet Eigenschaften einer AlltagserzäHlung (Quasthoff 1980, 1981; Gülich 1980, 2008; Kotthoff 2011; Schumann et al. 2015) wie das pointierte Wiedergeben eines zurückliegenden Ereignisses und das prosodische Markieren fremder Rede. Ebenfalls Mitglied dieser Ensembleaktivität ist der Regisseur, der seine Einzelaktivität AUSPACKEN zugunsten einer Beteiligung an der Ensembleaktivität pausiert, sowie die Schauspielerin, die in einem längeren Beitrag von einem Gespräch mit ihrer Mutter ERZÄHLT. Beide Teilnehmer zeigen ihr mithilfe von Blickkontakt, Lächeln und Lachpartikeln als engagement display an, dass sie sich als Beteiligte der ALLTAGSERZÄHLUNG verstehen. Die Schauspielerin reagiert auf diese Involvierungsanzeigen und wechselt ihren Blick zwischen den Interaktionspartnern. 
Tab. 13: Fortsetzen der pausierten Einzelaktivität bei Abbruch der Ensembleaktivität durch SCM.

\begin{tabular}{|c|c|c|c|c|}
\hline Transkript 9 & a) & b) & c) & d) \\
\hline \multirow[t]{2}{*}{$\begin{array}{l}\text { Einzelakti- } \\
\text { vität: JACKE } \\
\text { ABLEGEN }\end{array}$} & engag. display & Fortsetzungsproj. & Fortsetzungsproj. & $\begin{array}{l}\text { Fortsetzung nach } \\
\text { Relevanzverände- } \\
\text { rung }\end{array}$ \\
\hline & $\begin{array}{l}\text { haptische Betei- } \\
\text { ligungsweise }\end{array}$ & $\begin{array}{l}\text { haptische } \\
\text { Bindung }\end{array}$ & $\begin{array}{l}\text { haptische } \\
\text { Bindung }\end{array}$ & $\begin{array}{l}\text { hap. \& vis. } \\
\text { Beteiligungsweise }\end{array}$ \\
\hline Ensemble- & & engag. display & engag. display & \\
\hline aktivität: & & - & - & \\
\hline AlLtAGSER- & & vis. \& verb. & Auflösen der & \\
\hline
\end{tabular}

Im Verlauf der Interaktion konkurrieren der Regisseur und der Schauspieler um das Rederecht und damit um die verbale Beteiligungsweise an der Ensembleaktivität. Der Schauspieler verliert diesen Wettstreit. In der Folge zieht er sich aus der Ensembleaktivität zurück, indem er seine visuelle und verbale Beteiligung von ihr abzieht und seine pausierte haptische Beteiligungsweise der Einzelaktivität JACKE ABLEGEN fortsetzt (Tab. 13). Der Transkriptausschnitt setzt damit ein, wie die Schauspielerin erzählt, ihre Mutter habe eine plötzliche Meinungsänderung zu Kinderoperationen gehabt, die sie nun nicht mehr ab 10 Jahren, sondern sofort nach der Geburt durchführen lassen wollen würde.

\section{Transkript 9: Fortsetzung der pausierten Einzelaktivität JACKE ABLEGEN bei Rückzug aus Ensembleaktivität ALLTAGSERZÄHLUNG}

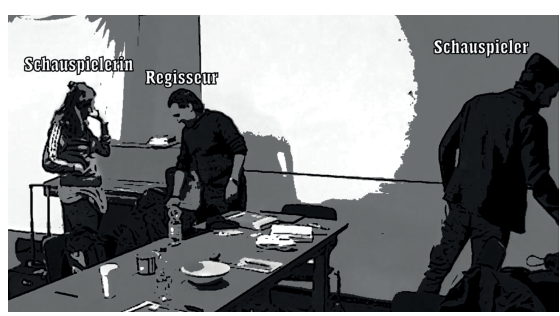

Abb. 9.1: SCW ERZÄHLT REG \& SCM von ihrer Mutter.

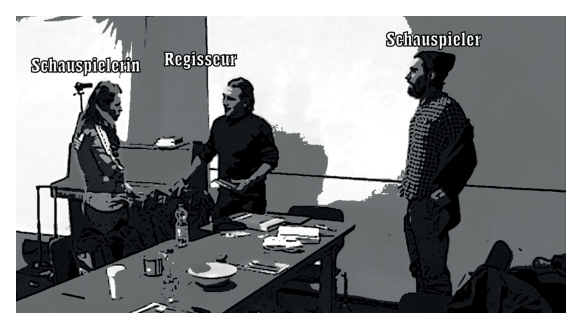

Abb. 9.2: REG und SCM pausieren ihre Einzelaktivitäten.

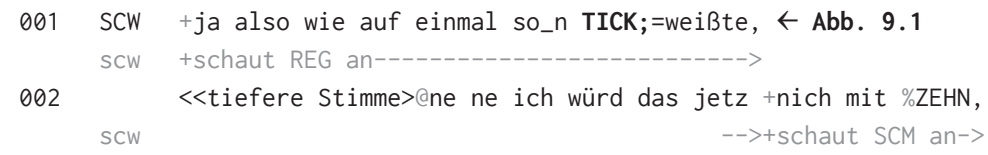


$\mathrm{scm}$

reg

\section{aschaut SCW an-}

$\%$ schaut SCW an $-\rightarrow>$

und ich würd das jetz (--)+direkt

nach der geBURT? $(--) \leftarrow$ Abb. 9.2

kein SCHMERZempfinden, und da TUST du der menschheit noch was GUtes mit;>

$+(2.0)+(2.0)$

+schaut REG an+ schaut SCM an+

REG aber is doch\% GUT;

reg $-->\%$ schaut auf Tisch->

SCW ALso-

reg \%schaut SCW an->

[aber die HAT_s- ]

$a b[$ er hast du sie ge]+FRAGT,

schaut auf Tisch---->+schaut SCM an->

[sach (---) ]

[was SAGT se denn, ]

[ja mama (.) + WICHtig, ] $\leftarrow$ Abb. 9.3

[ja WENN man (-)@ ?äh- ] Abb. 9.4

-->@schaut auf Kaffeekanne->

SCW ja sie hat noch mal geFRAGT,

und ich hab halt geSAGT,

dass es halt sehr komPLEX ähm is,

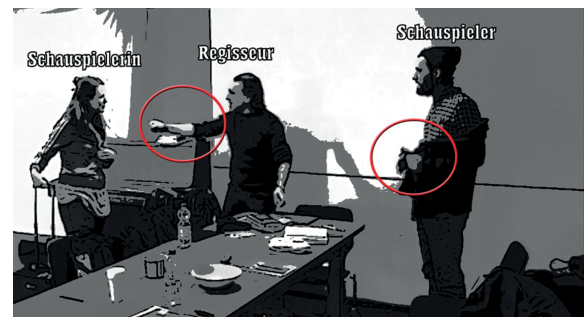

Abb. 9.3: REG und SCM gestikulieren.

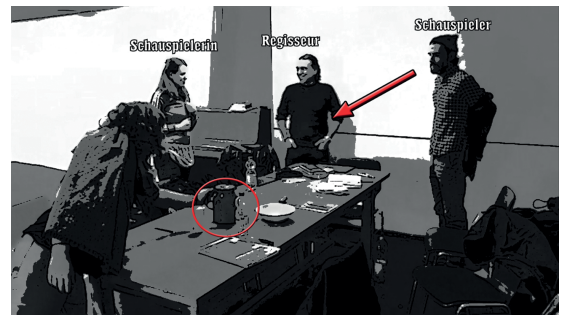

Abb. 9.4: SCM orientiert sich auf die Kaffeekanne.

a) engagement display bzgl. der Einzelaktivität via haptischer Beteiligungsweise (Z.001-002): Die Schauspielerin bewertet eine überraschende Einstellungsänderung ihrer Mutter als „TICK“ (Z.001), was sie mit einer vor ihrem Kopf schüttelnden Handbewegung illustriert (Abb. 9.1). Sie schaut dabei den Regisseur an, der den Blickkontakt nicht erwidert, sondern auf seine Tasche orientiert ist, in der er im Rahmen seiner AuSPACK-Einzelaktivität mit seiner rechten Hand tastet. 
Der Schauspieler ist der Schauspielerin mit dem Rücken zugewandt und legt seinen Mantel über einen Stuhl (Abb. 9.1). Obwohl keiner ihrer Interaktionspartner ihr Involvierung in die von ihr initiierte Ensembleaktivität anzeigt, bedient sich die Schauspielerin keiner (Äußerungs-)Pausen oder (Äußerungs-) Neustarts (Goodwin 1980a), um eine Orientierung auf die Ensembleaktivität zu elizitieren. ${ }^{39}$ Als die Schauspielerin mit prosodisch modulierter Stimme als „narrativem Effekt“ (Kotthoff 2011) die fremde Rede ihrer Mutter wiedergibt (Z.002), dreht sich zunächst der Schauspieler um, der sich auf die Schauspielerin und damit erkennbar auf die Ensembleaktivität orientiert und dabei seine Jacke AusZIEHT. Nur knapp danach hebt auch der Regisseur seinen Kopf, nimmt Blickkontakt mit der Schauspielerin auf und holt unterdessen zwei Notizbücher aus seiner Tasche.

b) Pausieren der Einzelaktivität durch Fortsetzungsprojektion via haptischer Bindung mit dem Fokusobjekt bei gleichzeitigem engagement display bzgl. der Einzelaktivität via visueller und verbaler Beteiligungsweisen (Z.003-011): Mit der Wiedergabe der Rede der Mutter, diese würde die Operation „direkt nach der geBURT“ (Z.003) durchführen, pausieren Regisseur und Schauspieler beide nahezu simultan ihre Einzelaktivitäten, indem beide in ihrer Gestalt einfrieren: Der Regisseur hält weiterhin die Notizbücher mit der linken Hand, während die rechte Hand die Tasche offenhält. Der Schauspieler hat derweil die Jacke in der Kernphase der Aktivität auf „halbe Höhe“ heruntergezogen (Abb. 9.2). Das Pausieren ist an dieser Stelle möglich, da weder Regisseur noch Schauspieler für den Vollzug der Einzelaktivitäten Beteiligungsweisen verwenden, die auch in der Ensembleaktivität eingesetzt werden könnten. Die haptische Beteiligungsweise ist damit strukturell kompatibel mit der visuellen und der verbalen Beteiligungsweise der Ensembleaktivität. Die pausierte Einzelaktivität gibt den Interagierenden die Möglichkeit, Blickkontakt mit anderen Beteiligten der Ensembleaktivität aufzunehmen. In der analysierten Sequenz orientieren sich Regisseur und Schauspieler jeweils auf die erzählende Schauspielerin und konkurrieren um den Blickkontakt mit ihr. Eine Orientierung aufeinander bzw. Blickkontakt miteinander findet nicht statt. Beide pausieren ihre Einzelaktivitäten nahezu gleichzeitig und zeigen damit unabhängig voneinander eine veränderte Relevanz der als abgeschlossen behandelten ERZÄHLUNG an, die nun auch verbale Beteiligungen der Zuhörenden ermöglicht. Diese pausierte Gestalt der Einzelaktivitäten halten beide Interagierende bis zum Ende der Redewiedergabe (Z.005) und

39 Möglicherweise waren die vorherigen lachenden und lächelnden Reaktionen von Regisseur und Schauspieler Beteiligungsanzeige genug, damit die Schauspielerin an dieser Stelle trotz engagement display bzgl. in die jeweiligen Einzelaktivitäten eine Involvierung in die Ensembleaktivität annehmen kann. 
anschließenden verbalen Pause (Z.006) aufrecht. In dieser 4-sekündigen Phase verbaler Abstinenz schaut die Schauspielerin zunächst den Regisseur und danach den Schauspieler an. Sie evoziert damit eine Bewertung ihrer Erzählung, die durch den Regisseur eingelöst wird. Seine pausierte Gestalt aufrechterhaltend realisiert dieser eine positive Bewertung ,aber is doch GUT“ (Z.007). Als die Schauspielerin beginnt, darauf verbal einzugehen (Z.008) und sich kurz von ihren beiden Interaktionspartnern abwendet, nutzt der Regisseur diesen unterbrochenen Blickkontakt dafür, seine pausierte Einzelaktivität wiederaufzunehmen, die Notizbücher abzulegen und die Einzelaktivität AuSPACKEN abzuschließen (Z.007). Damit lässt sich an dieser Stelle die Funktion der Pausierung ableiten: Der Regisseur pausiert die Einzelaktivität genau an der Stelle, an der er neben der haptischen auch auf eine visuelle Beteiligungsweise zurückgreifen muss, um den Ort zu lokalisieren, an dem er das AUSGEPACKTE Objekt ablegen kann. Da diese visuelle Beteiligungsweise mit der visuellen Beteiligung an der Ensembleaktivität strukturell inkompatibel ist, stellt der Regisseur diese Beteiligungsweise und damit den Vollzug der Aktivität solange zurück, bis die visuelle Beteiligungsweise im Rahmen der Ensembleaktivität (kurzzeitig) nicht benötigt wird. Im Gegensatz dazu behält der Schauspieler seine pausierte Gestalt und damit auch seine visuelle Beteiligungsweise bei, wodurch er anzeigt, dass er die Ensembleaktivität situativ in Bezug zu seiner Einzelaktität priorisiert. Neben der visuellen Beteiligungsweise versucht der Schauspieler auch im Rahmen der verbalen Beteiligungsweise fragend an der Ensembleaktivität teilzunehmen. Allerdings überlappt er zunächst mit der Schauspielerin (Z009/10), woraufhin er die Frage reformuliert (Z.012), die aber diesmal mit einer Äußerung des Regisseurs überlappt (Z.011).

c) Auflösen des engagement displays bzgl. der Ensembleaktivität via Abzug der verbalen und visuellen Beteiligungsweisen bei fortbestehendem Pausieren der Einzelaktivität via haptischer Bindung mit dem Fokusobjekt (Z.012-14): Auch der dritte Versuch des Schauspielers, sich verbal an der Ensembleaktivität zu beteiligen, resultiert in einer Überlappung mit dem Regisseur (Z013/14). Regisseur und Schauspieler konkurrieren somit an dieser Stelle um das Rederecht und um den Blickkontakt mit der Schauspielerin. Beide Konkurrierenden wählen die gleiche Strategie, um Rederecht und Blickkontakt zu erhalten, indem beide anfangen zu gestikulieren. Allerdings fällt die Geste des Schauspielers, der weiterhin seine Jacke im Rahmen seiner AuszIEH-Aktivität festhält, deutlich kleiner aus, als die einem Faustschlag ähnliche Handbewegung des Regisseurs (Abb. 9.3). Die Schauspielerin wendet daraufhin den Blick vom Schauspieler ab und orientiert sich auf den Regisseur. Der Schauspieler kommentiert den verlorenen Wettstreit um das Rederecht mit einem durch Glottalverschluss auffälligen change-of-state token (Heritage 1984; Imo 2009) „?äh“(Z.014), noch bevor 
der Regisseur seine TCU abgeschlossen hat. Der Schauspieler zeigt damit an, dass er die Auswahl des nächsten Sprechers über den Blickkontakt als abgeschlossen betrachtet.

d) Fortsetzen der Einzelaktivität durch Wiederaufnahme der haptischen Beteiligungsweise bei disengagement display bzgl. der Ensembleaktivität durch visuelle Reorientierung (Z.015-017): Als die Schauspielerin ihre ALLTAGSERZÄHLUNG wiederaufnimmt (Z.015-17), wendet sich der Schauspieler per body torque von der Schauspielerin zur Regieassistentin, die eine Kaffeekanne auf den Tisch stellt, während er gleichzeitig seine pausierte AuszIEH-Einzelaktivität fortsetzt und auf diese Weise sein engagement display bzgl. der Ensembleaktivität AlltagsERZÄHLUNG auflöst (Abb. 9.4). Die Fortsetzung realisiert der Schauspieler hier also nicht, weil seine Einzelaktivität neue Relevanz gewonnen hätte, sondern weil die ko-relevante Ensembleaktivität aufgrund seiner verhinderten verbalen Beteiligung situativ weniger relevant für ihn wird. Er depriorisiert daraufhin die Ensembleaktivität und setzt seine Einzelaktivität fort. Die Fortsetzung der Einzelaktivität erfolgt damit ab dem Moment, ab dem sich der Schauspieler aus der Ensembleaktivität zurückzieht und damit seine Beteiligung an ihr abbricht. Die Fortsetzungsprojektion der „,auf halber Höhe“ gehaltenen Jacke wird eingelöst, sobald der Anlass zur Pausierung (=Möglichkeit der Beteiligung an der Ensembleaktivität) nicht mehr relevant ist. Ohne eine gleichzeitige Relevanz zweier Handlungsstränge ist es für den Schauspieler nicht mehr notwendig, multiple Aktivitäten zu koordinieren. Dies resultiert hier darin, dass er zu einem monoaktiven engagement display übergeht.

Eine zweite Form veränderter Relevanz in einer sozialen Interaktion, die auch bei multiplen Aktivitäten beobachtet werden und eine Auflösung einer Fortsetzungsprojektion nach sich ziehen kann, ist das Einlösen einer konditionellen Relevanz. Als konditionelle Relevanz wird in der Konversationsanalyse die Erwartbarkeit einer zweiten Handlung nach einer bestimmten ersten Handlung bezeichnet:

By conditional relevance of one item on another we mean: given the first, the second is expectable; upon its occurrence it can be seen to be a second item to the first; upon its nonoccurrence it can be seen to be officially absent.

(Schegloff 1968: 1083)

Der erste Paarteil einen Vorschlag unterbreiten der Ensembleaktivität VoRSCHLAGEN macht einen zweiten Paarteil den Vorschlag bearbeiten (z. B. Vorschlag annehmen/ablehnen oder Vorschlag bewerten) erwartbar. Bleibt der zweite Paarteil gänzlich aus, wird sein Nichtauftreten bearbeitet, wie im folgenden Tran- 
skriptausschnitt. ${ }^{40}$ In diesem expandiert die Schauspielerin ihren VoRSCHLAG, das gemeinsame Kind der Figuren solle sterben. Während sie per visueller und verbaler Beteiligungsweisen den ersten Paarteil des VoRschlAGs bearbeitet, hält sie ihre Mütze in der Hand und damit die Fortsetzungsprojektion ihrer pausierten Einzelaktivität KLEIDUNG ABLEGEN per haptischer Bindung aufrecht. Sie setzt die pausierte Aktivität fort, als die ratifizierten Beteiligten ${ }^{41}$ der Ensembleaktivität (Regieassistentin und Videograph) den zweiten Paarteil nicht realisieren. Die Schauspielerin interpretiert dies als Account für die Ablehnung ihres VoRSCHLAGS und löst die Fortsetzungsprojektion ihrer pausierten Einzelaktivität ein, indem sie diese per haptischer Beteiligungsweise realisiert (Tab. 14). Das Fortsetzen der pausierten (und damit relevant gehaltenen) Aktivität zeigt eine Veränderung der Ensembleaktivität an. Diese Statusänderung ergibt sich hier aus der Einlösung bzw. Bearbeitung der konditionellen Relevanz durch die Beteiligten. Damit findet auch in diesem Beispiel eine Relevanzverschiebung innerhalb der multiplen Aktivitäten von der priorisierten Ensembleaktivität zur Einzelaktivität statt.

Tab. 14: Fortsetzen der pausierten Einzelaktivität bei Einlösen der konditionellen Relevanz der Ensembleaktivität durch SCW.

\begin{tabular}{|c|c|c|}
\hline Transkript 10 & a) & b) \\
\hline $\begin{array}{l}\text { Einzelaktivität: KLEIDUNG } \\
\text { ABLEGEN }\end{array}$ & $\begin{array}{l}\text { Fortsetzungsprojektion } \\
\text { haptische } \\
\text { Bindung }\end{array}$ & 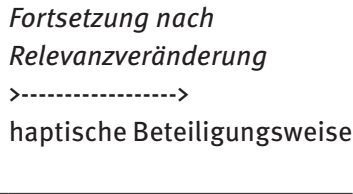 \\
\hline $\begin{array}{l}\text { Ensembleaktivität: } \\
\text { VoRSCHLAGEN }\end{array}$ & $\begin{array}{l}\text { engagement display } \\
-------> \\
\text { visuelle \& verbale } \\
\text { Beteiligungsweisen }\end{array}$ & $\begin{array}{l}\text { engagement display } \\
\text {---.----.--> } \\
\text { visuelle } \& \text { verbale } \\
\text { Beteiligungsweisen }\end{array}$ \\
\hline
\end{tabular}

Im Unterscheid zum vorherigen Transkript 9 tritt diese Veränderung innerhalb des lokalen Relevanzsystems der gleichzeitig relevanten Aktivitäten allerdings nicht nach einer verhinderten Beteiligung an einer Aktivität auf, sondern findet nach Ausbleiben einer erwartbaren Beteiligung durch die Mitglieder des Interaktionsensembles statt.

40 Es handelt sich hier um denselben VoRSCHLAG, der bereits in Transkript 8 untersucht wurde. Zwischen Transkript 8 und Transkript 10 liegen etwa 20 Sekunden, in denen die Schauspielerin ihren VoRSCHLAG weiter ausführt.

41 Der ebenfalls anwesende Hospitant ist seit mehreren Minuten in seine Laptop-Einzelaktivität involviert und wird von den anderen Teilnehmenden nicht adressiert. 


\section{Transkript 10: Fortsetzung der pausierten Einzelaktivität KLEIDUNG ABLEGEN nach Einlösung der konditionellen Relevanz der Ensembleaktivität VORSCHLAGEN}

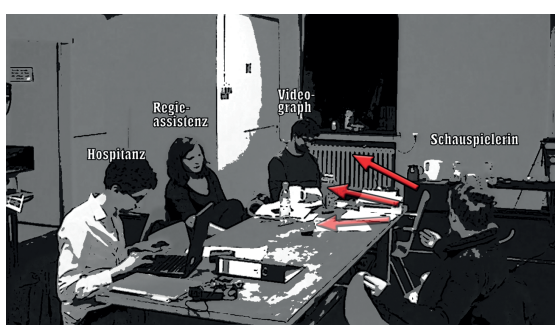

Abb. 10.1: SCW schaut die Beteiligten an.

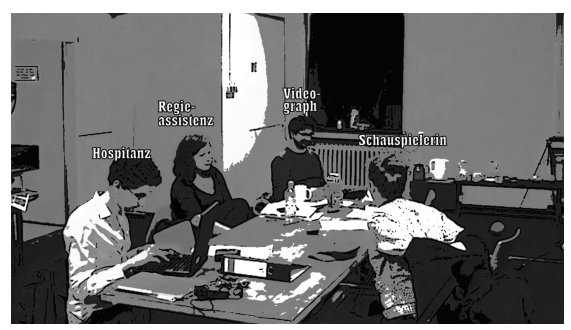

Abb. 10.2: SCW zieht Jacke aus.

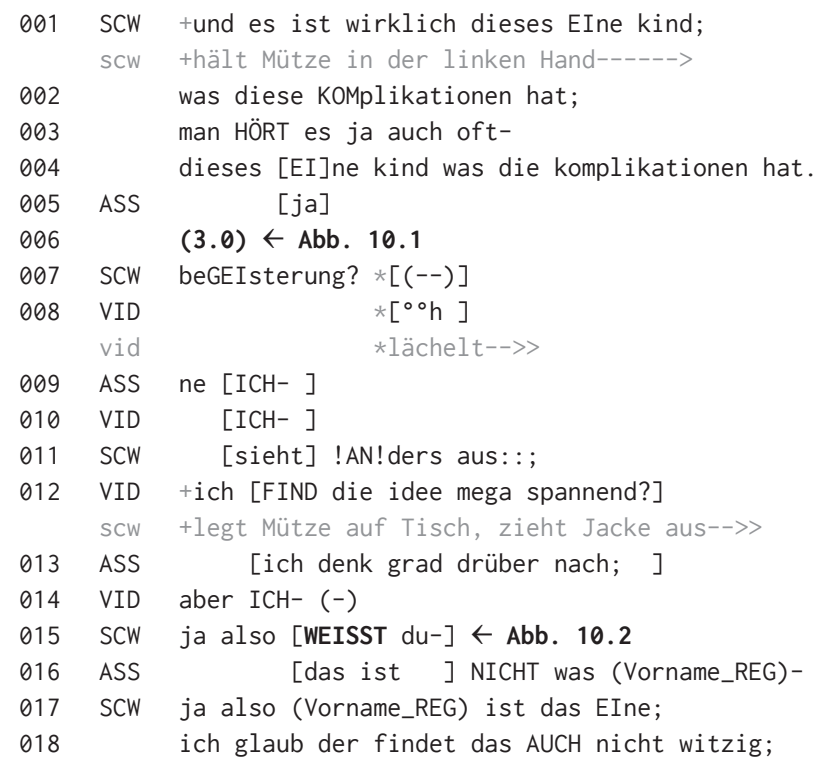

a) Fortsetzungsprojektion der pausierten Einzelaktivität via haptischer Bindung mit dem Fokusobjekt bei gleichzeitigem engagement display bzgl. der Ensembleaktivität via visueller und verbaler Beteiligungsweisen (Z.001-011): Die Schauspielerin schließt die Erläuterung ihres VoRschLAGs ab und markiert das Ende mittels stark fallender Intonation (Z.004) sowie einer längeren verbalen Pause (Z.006). Nachdem zuvor lediglich die Regieassistentin ein engagement display via verbaler (Z.005) sowie visueller Beteiligungsweise (Abb. 10.1) bzgl. der Ensembleaktivität angezeigt hat, nutzt die Schauspielerin die Pause, um nachei- 
nander den Videographen, die Regieassistentin und den Hospitanten anzuschauen (Abb. 10.1). Als am Ende ihres schweifenden Blicks keine Bearbeitung des Vorschlags durch die visuell Adressierten erfolgt, bewertet sie das „nicht begeisterte“ (Z.007/11) Verhalten der Anwesenden als Ablehnung ihres VorSCHLAGS.

b) Fortsetzung der pausierten Einzelaktivität via haptischer Beteiligungsweise bei gleichzeitigem engagement display bzgl. der Ensembleaktivität via visueller und verbaler Beteiligungsweisen (Z.012-018): Als Folge ihrer Interpretation des Verhaltens von Regieassistentin und Videograph legt die Schauspielerin ihre Mütze ab, beginnt ihre Jacke auszuziehen und nimmt dadurch ihre in der Endphase pausierte AUSZIEH-Einzelaktivität wieder auf (Abb. 10.2). Mit ihrer verbalisierten Interpretation der ausbleibenden Reaktion elizitiert sie Accounts von Regieassistentin und Videographen, die verbal überlappend auf die Kritik der Schauspielerin bzgl. der ausgebliebenen Reaktion auf ihren VoRSCHLAG reagieren und damit anzeigen, dass für sie die VoRsCHLAGs-Ensembleaktivität noch andauert (Z.009-013). Während die Regieassistentin ihren mentalen Status kommentiert (,ich denk grad drüber nach“, Z.013) und damit als kognitive Einzelaktivität accountable macht, liefert der Videograph eine positive Bewertung („ich FIND die idee mega spannend“, Z.012), für die er eine Einschränkung projiziert (,aber ICH“, Z.014). Auch die Regieassistentin meldet Bedenken an, ob dieser Vorschlag im Sinne der Vision des Regisseurs sei (Z.016). Dies führt dazu, dass die Schauspielerin in den nächsten zwei Minuten ihren VoRschlag erneut ausführt, die inszenatorischen Möglichkeiten darstellt und gemeinsam mit der Regieassistentin und dem Videographen (sowie etwas später auch mit der Hospitantin, siehe Transkript 7) die dramaturgischen Implikationen des VoRSCHLAGS diskutiert.

Der Fall illustriert, dass sowohl das Pausieren wie auch das Fortsetzen von Einzelaktivitäten nicht willkürlich, sondern funktional an interaktional dafür passenden Slots geschehen kann. Als auf das Unterbreiten des Vorschlags der Schauspielerin kein zweiter Handlungsteil Bearbeiten des Vorschlags durch die Anwesenden folgt, fordert die Schauspielerin eine aktive Beteiligung der Ensembleaktivität ein. Das Ausbleiben einer solchen Beteiligung interpretiert sie als Ablehnung ihres VoRschlags und nimmt ihre während der Handlung Vorschlag unterbreiten pausierte AuszIEH-Aktivität wieder auf. Das Fortsetzen der pausierten (und damit relevant gehaltenen) Aktivität zeigt somit eine Veränderung im Status der Ensembleaktivität und damit im lokalen Relevanzsystem der multiplen Aktivitäten an. Diese Statusänderung ergibt sich aus dem Einlösen bzw. dem Bearbeiten der konditionellen Relevanz durch die Beteiligten. Ein vergleichbarer 
Fall ist in der Analyse von Transkript 7 (SCM pausiert sein RUCKSACKABSETZEN) bereits beschrieben worden, wo sich die Umsetzung der Fortsetzungsprojektion ebenfalls nach Einlösen der konditionellen Relevanz beobachten ließ. Im Gegensatz zum Beispiel in Transkript 10 hält dort aber der Schauspieler seine Einzelaktivität AUSPACKEN durch Festhalten der Rucksackgurte solange pausiert, bis der VORSCHLAG von den Beteiligten als angenommen behandelt wird und die Schauspielerin eine neue Ensembleaktivität initiiert.

Ein ähnlicher Fall findet sich im folgenden Transkript $11 .^{42}$ Hier hält der Hospitant seine pausierte Einzelaktivität TRINKEN pausiert, bis die Ensembleaktivität ERKLÄREN einen abgeschlossenen Status erhält. Im Gegensatz zu den vorherigen Fällen ist es hier aber die Fortsetzung der Einzelaktivität (nicht der Ensembleaktivität), die die Ensembleaktivität als abgeschlossen markiert, da der Hospitant derjenige mit der verbalen Beteiligung an der Ensembleaktivität ist.

Tab. 15: Fortsetzen der pausierten Einzelaktivität bei Abschluss der Ensembleaktivität durch HOM.

\begin{tabular}{|c|c|c|c|c|}
\hline Transkript 11 & a) & b) & c) & d) \\
\hline $\begin{array}{l}\text { Einzelaktivi- } \\
\text { tät: TRINKEN }\end{array}$ & & $\begin{array}{l}\text { engag. display } \\
\text {-yis. \& hap. } \\
\text { Beteiligungsw. }\end{array}$ & $\begin{array}{l}\text { Fortsetzungsproj. } \\
\text { haptische Bindung }\end{array}$ & 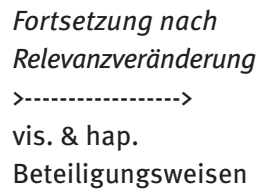 \\
\hline $\begin{array}{l}\text { Ensemble- } \\
\text { aktivität: } \\
\text { ERKLÄREN }\end{array}$ & $\begin{array}{l}\text { engag. display } \\
\text { vis. \& verb. } \\
\text { Beteiligungsw. }\end{array}$ & & $\begin{array}{l}\text { engag. display } \\
\text { vis. \& verb. } \\
\text { Beteiligungsw. }\end{array}$ & \\
\hline
\end{tabular}

Wie Hoey (2018a) bereits zeigen konnte, verhält sich die TRINK-Aktivität strukturell inkompatibel zu allen Aktivitäten, für die Teilnehmende auf verbale Beteiligungsweisen zur Realisierung zurückgreifen. Es wäre damit durchaus erwartbar, dass der Hospitant eine der gleichzeitig relevanten, aber strukturell in Bezug auf die Beteiligungsweisen inkompatiblen Aktivitäten TRINKEN und ERKLÄREN zugunsten der anderen abbricht. Dass es hier nicht zu einem Abbruch, sondern einem Pausieren der Trink-Aktivität durch den Hospitanten kommt, hängt damit

42 Die in Transkript 11 analysierte Sequenz ist bereits in Transkript 2 in Bezug auf den Abbruch der Ensembleaktivität besprochen worden. 
zusammen, wann der Hospitant die Aktivität pausiert und welche Fortsetzungsprojektion er zum Aufrechterhalten der Relevanz verwendet (Tab. 15).

\section{Transkript 11: Fortsetzung der pausierten Einzelaktivität TRIN KEN bei} gemeinsamem Abschluss der Ensembleaktivität ERKLÄREN
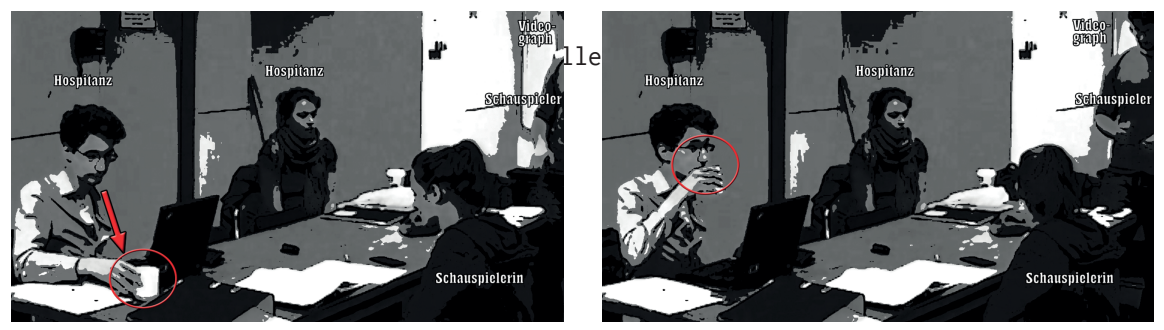

Abb. 11.1: HOM greift die Tasse.

Abb. 11.2: HOM führt die Tasse zum Mund.

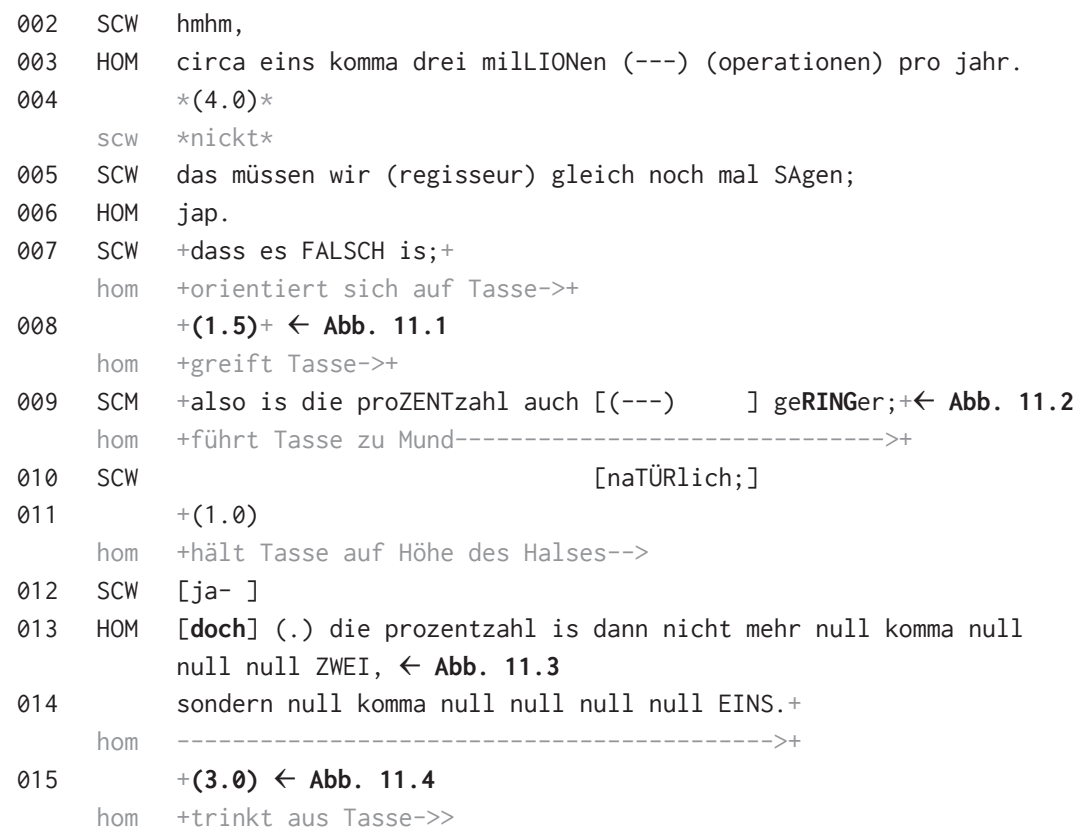




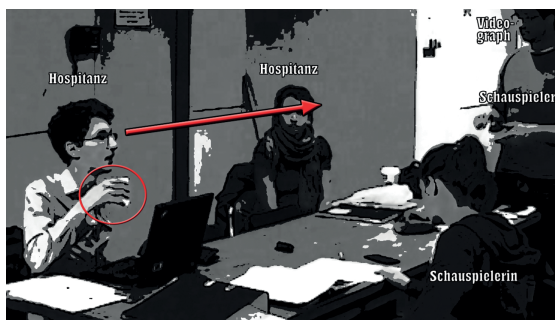

Abb. 11.3: HOM hält die Tasse in working position.

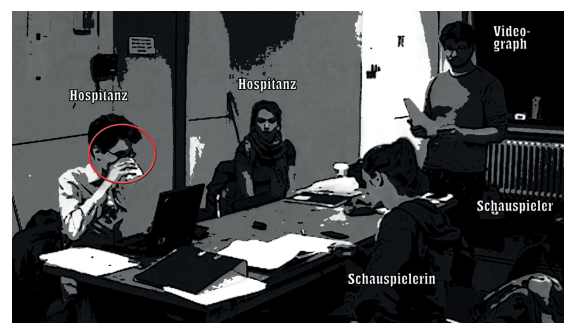

Abb. 11.4: HOM TRINKT aus der Tasse.

a) engagement display bzgl. der Ensembleaktivität via visueller und verbaler Beteiligungsweisen (Z.001-006): Von den fünf Anwesenden (Hospitant, Hospitantin, Videograph, Schauspielerin und Schauspieler) stellen nur die Schauspielerin und der Hospitant gemeinsam eine Ensembleaktivität her. Sie schauen einander an, sind aufeinander orientiert und geben einander Rezipientensignale (Z.002), während der Hospitant einen von ihm korrigierten Fakt zusammenfasst (Z.001-3), den er zuvor ERKLÄRT hat. Der Blickkontakt wird in einer 4-sekündigen Phase verbaler Abstinenz aufrechterhalten, in der die Schauspielerin langsam nickt (Z.004). Schauspielerin und Hospitant bringen ihre Ensembleaktivität dadurch gemeinsam zu einem Abschluss, indem die Schauspielerin die gemeinsame Handlungsanweisung ableitet, den Regisseur von der falschen Information im Textbuch in Kenntnis zu setzen (Z.005-7), die vom Hospitanten bestätigt wird (Z.006).

b) engagement display bzgl. der Einzelaktivität via visueller und haptischer Beteiligungsweisen (Z.007-010): Nach Realisierung seiner Bestätigung ändert der Hospitant sein engagement display. Er senkt den Kopf, löst dadurch nach der verbalen auch die visuelle Beteiligung an der Ensembleaktivität auf und greift seine Tasse, um die Startphase der Trink-Einzelaktivität zu initiieren (Abb. 11.1). Auch die Schauspielerin zeigt ihren Ausstieg aus der Ensembleaktivität an, indem sie eine Einzelaktivität startet. Sie senkt dafür ebenfalls den Kopf und orientiert sich auf ihr geöffnetes Textbuch vor ihr, um (möglicherweise) eine LESE-Aktivität zu starten. Etwa anderthalb Sekunden vergehen, ohne dass eine ${ }^{\star}$ r der Anwesenden eine Involvierung in die Ensembleaktivität anzeigt, die dadurch einen abgeschlossenen Status erhält. Daraufhin nimmt der Schauspieler die Ensembleaktivität wieder auf, indem er das verhandelte Thema wiederaufgreift und auf die falsche Information (,die proZENTzahl“, Z.009) verweist. Er liefert mit der Verwendung von „also“ eine Verstehensinferenz (Deppermann \& Helmer 2013) und orientiert sich mit Blickkontakt sowie body torque (Schegloff 1998) auf den Hospitanten hin. Dieser führt während der Äußerung des Schauspielers seine Tasse 
zum Mund und reagiert auf diese Adressierung, indem er seinen Blick auf den Schauspieler richtet (Abb. 11.2). Die Schauspielerin indes löst den Blick von ihrem Textbuch und bestätigt die Schlussfolgerung des Schauspielers verbal (Z.010).

c) Pausieren der Einzelaktivität durch Herstellen einer Fortsetzungsprojektion via haptischer Bindung mit dem Fokusobjekt bei gleichzeitigem engagement display bzgl. der Ensembleaktivität via visueller und verbaler Beteiligungsweisen (Z.011014): Bevor der Hospitant einen Schluck aus seiner Tasse nehmen und damit in die Kernphase seiner Einzelaktivität übergehen kann (was eine strukturelle Inkompatibilität der Beteiligungsweisen nach sich ziehen würde), hält er in seiner Bewegung inne und senkt die Tasse auf Höhe seines Halses ab (Abb. 11.3). Dadurch wird es ihm möglich, sich verbal an der wiederaufgenommenen Ensembleaktivität $\mathrm{zu}$ beteiligen und gleichzeitig seine Einzelaktivität in der working postion (Schegloff 1998) relevant zu halten. Sein darauffolgender Interaktionsbeitrag überlappt mit einer weiteren Äußerung der Schauspielerin, die ihren Turn daraufhin abbricht (Z.012/13). Blickkontakt mit dem fragenden Schauspieler aufrechterhaltend bestätigt der Hospitant die Schlussfolgerung des Schauspielers und liefert Informationen zur falschen wie zur korrekten Prozentzahl (Z.013/14).

d) Fortsetzung der pausierten Einzelaktivität via haptischer Beteiligungsweise (Z.015): Nachdem der Hospitant seine ERKLÄRUNG syntaktisch und prosodisch als abgeschlossen markiert hat, löst er den Blickkontakt auf, führt seine immer noch kurz vor seinem Mund gehaltene Tasse zurück an seine Lippen und realisiert die Kernphase seiner TRINK-Einzelaktivität (Abb. 11.4). Das Pausieren und Fortsetzen der TRINK-Aktivität durch den Hospitanten bei gleichzeitiger Bearbeitung der Relevanz der strukturell damit inkompatiblen ERKLÄR-Aktivität wird hier dadurch möglich, dass der Hospitant die Start- und Kernphasen seiner TRINKEinzelaktivität in Bezug auf den Status der ko-relevanten Ensembleaktivität zeitlich abstimmt. Bei ihm ist eine Art situative „Vorplanung“ zu erkennen, als er das TRINKEN vor der Kernphase pausiert, um sich verbal an der Ensembleaktivität beteiligen zu können und nach seiner Beteiligung die Kernphase schließlich durchführt. Die Fortsetzungsprojektion erreicht er hier, indem er seine TRINK-Gestalt in eine working position überführt und sie solange dort „parkt“, bis sich die Relevanz der Ensembleaktivität verändert hat. Damit sind Fortsetzen und Pausieren hier nicht nur Praktiken, mit denen er auf die Anforderung gleichzeitiger Relevanz zweier unterschiedlicher Handlungsstränge reagiert, sondern ebenfalls eine multimodale Anzeigeleistung bzgl. der Relevanzveränderung der Ensembleaktivität. Während der erste Abschluss der Ensembleaktivität zwischen Hospitanten und Schauspielerin wechselseitig ausgehandelt wird, erfolgt die finale Abschlussmarkierung des zweiten Abschlusses mit Fortsetzung der pausierten 
Einzelaktivität. Indem der Sprechende durch die Ausführung der Einzelaktivität die Möglichkeit seiner verbalen Beteiligung an der Ensembleaktivität einschränkt, ist für die Ko-Interagierenden für die Dauer der Kernphase dieser Einzelaktivität davon auszugehen, dass keine weitere Beteiligung an dieser Ensembleaktivität erfolgen wird. Damit demonstriert das Beispiel, dass die gleichzeitige Relevanz multipler Aktivitäten nicht nur koordinative Anforderungen an die Teilnehmenden stellt, sondern dass die Interagierenden ihre engagement displays bzgl. der Aktivitäten auch dahingehend nutzen können, um interaktionale Aufgaben wie das Abschließen einer Ensembleaktivität zu bearbeiten.

Wie die Analysen dieses Abschnitts gezeigt haben, handelt es sich bei Pausierungen und Fortsetzungen von Aktivitäten um Koordinierungspraktiken, mit denen Interagierende gleichzeitige Relevanzen bearbeiten. Die Pausierung einer Aktivität kann bei einer multimodalen Beteiligung an der Ensembleaktivität zum Aufrechterhalten des Blickkontakts erforderlich sein (Transkript 6), nach einer Beteiligungseinforderung notwendig werden (Transkript 7) oder mit einer Beteiligungsrelevantsetzung einhergehen (Transkript 8). Das Relevanthalten einer pausierten Aktivität wird erreicht, indem die Teilnehmenden eine konstante Fortsetzungsprojektion leisten. Diese wird zum Ersten durch das Einfrieren der jeweiligen Beteiligungsweise in der Start- (Transkript 6 \& 7), Kern- (Transkript 8 \& 9) oder Endphase (Transkript 10) der zu pausierenden Einzelaktivität erzeugt. Möglich ist zum Zweiten auch das Überführen der Beteiligungsweise für die Dauer der Pausierung in eine temporäre working position (Transkript 11) der Einzelaktivität. Zum Dritten ermöglichen Sprechpausen ein Pausieren einer verbalen Beteiligung an einer Ensembleaktivität, wenn diese syntaktisch und prosodisch so in die TCUs eingebettet werden, dass sie eine Fortsetzung der verbalen Beteiligung erwartbar machen. Diese Fortsetzungsprojektion wird bei allen hier analysierten Aktivitätspausierungen als solche accountable gemacht und lässt die zeitnahe Fortsetzung der pausierten Aktivität für die Teilnehmenden erkennbar werden. Fortsetzungen erfolgen in den besprochenen Sequenzen an solchen Stellen, an denen sich die gleichzeitige Relevanz, die eine Pausierung zuvor notwendig werden ließ, aufgelöst hat. Dies umfasst Situationen, in denen die verbale Beteiligung an einer Ensembleaktivität verweigert (Transkript 9), die konditionelle Relevanz einer Ensembleaktivität eingelöst (Transkript 10) oder eine Ensembleaktivität gemeinsam abgeschlossen wird (Transkript 11). Voraussetzung von Pausierungen im Rahmen gleichzeitiger Relevanz multipler Aktivitäten ist eine zumindest teilweise strukturelle Kompatibilität der verwendeten Beteiligungsweisen. Dies zeigt sich deutlich an Transkript 9. Dort kann der Regisseur an einer ALLTAGSERZÄHLUNG mit verbalen und visuellen Beteiligungsweisen partizipieren, während er mittels haptischer Beteiligungsweise seine zwei Notiz- 
bücher in der Hand und seine Einzelaktivität AUSPACKEN dadurch pausiert hält. Dass sich die beiden für ihn ko-relevanten Aktivitäten ALLTAGSERZÄHLUNG und AUSPACKEN nicht völlig strukturell kompatibel zueinander verhalten, zeigt er, als er an einem passenden Slot (Abbruch des Blickkontakts der Sprecherin) die visuelle Beteiligungsweise von der AlltAGsERzÄHLung abzieht und sie dafür nutzt, um im Rahmen des AusPACKENS einen Ablageort für seine Notizbücher zu lokalisieren. Damit sind die beiden ko-relevanten Aktivitäten in dieser Situation zwar nicht strukturell inkompatibel, teilen sich aber im Kern zum Vollzug dieselbe (visuelle) Beteiligungsweise. Da sich die Beteiligungsweisen jedoch nicht gegenseitig ausschließen, muss der Regisseur keine der Aktivitäten abbrechen, sondern pausiert eine der Aktivitäten lediglich, bis die benötigte Beteiligungsweise frei wird. Pausierungen und Fortsetzungen ermöglichen es Interagierenden also die gleichzeitige Relevanz multipler Aktivitäten so zu bearbeiten, dass eine Beteiligung an mehreren Aktivitäten simultan möglich wird. Denn anders als bei den Praktiken Abbrüche und Wiederaufnahmen der seriellen Koordination (5.1), die die Relevanz der abgebrochenen Aktivitäten im Vordergrund auflösen, zeigen Interagierende mithilfe der Fortsetzungsprojektion die stete Relevanz der pausierten Aktivität an. Die dadurch entstehende Koordinierungsform ist dabei nur quasi-simultan, da im Vergleich zur simultanen Koordinierung (5.3) zwar ein durchgängiges engagement display angezeigt wird, aber keine stete Bearbeitung der Aktivität erfolgt.

\subsection{Praktiken der simultanen Koordination: Singuläre und multiple Beteiligungsmarker}

Wenn Interagierende keine der multiplen Aktivitäten zugunsten einer anderen beenden, abbrechen oder pausieren und stattdessen ihre multiplen Aktivitäten dergestalt vollziehen, dass die Aktivitäten gleichzeitig realisiert werden können, entstehen Aktivitätskomplexe, die simultan koordiniert werden. Solche simultan koordinierten Multiaktivitäten sind interaktionale Einheiten, die aus mehreren Aktivitäten bestehen, deren simultane Relevanz gleichzeitig bearbeitet wird und bei denen dies im engagement display sichtbar wird. Eine Voraussetzung dafür ist die emergente „Transformation der Gleichzeitigkeit“ (Pitsch 2006: 198-199), bei der multimodale Ressourcen so auf die Teil-Aktivitäten einer Multiaktivität aufgeteilt werden, dass die gleichzeitige Bearbeitung multipler Relevanzen einerseits möglich und andererseits für andere Teilnehmende erkennbar wird. Wie die bisherigen Analysen gezeigt haben, stellen die Teilnehmenden je nach strukturaler Kompatibilität der verwendeten Beteiligungsweisen und Priorisierung der Aktivitäten im lokalen Relevanzsystem der vollzogenen Aktivitäten unterschiedliche 
engagement displays her, auf die Ko-Interagierende reagieren können. Die folgenden Analysen zeigen, dass innerhalb des Interaktionsensembles bereits eine einzelne Beteiligungsweise ausreicht, damit Teilnehmende ein engagement display bzgl. einer Aktivität annehmen. Solche singulären Beteiligungsmarker zur simultanen Involvierung in multiple Aktivitäten (5.3.1) können verbal abstinente Beteiligungsweisen des Nickens und des Blickkontakts (Transkript 12) als auch Rückmeldepartikeln ohne Blickkontakt (Transkripte 13 \& 14) umfassen. Bei singulären Beteiligungsmarkern kann es allerdings trotz entstehendem dualen engagement display bzgl. der ko-relevanten Aktivitäten vorkommen, dass Teilnehmende in ihren Reaktionen anzeigen, dass ihnen die singuläre Beteiligungsanzeige zwar ausreicht, sie aber dennoch eine Störung im Interaktionsfluss - beispielsweise das Fehlen eines Blickkontakts - bearbeiten. Im Vergleich dazu sind engagement displays deutlich robuster, wenn Interagierende auf multiple Beteiligungsmarker der simultanen Involvierung in multiple Aktivitäten (5.3.2) zurückgreifen. Damit wird es Interagierenden möglich, nicht nur die Teilnahme an mehreren Aktivitäten öffentlich im engagement display anzuzeigen, sondern auch die Relevanzen multipler Aktivitäten aktiv zu bearbeiten. Solche Multiaktivitäten können entweder aus Kombinationen von Ensemble- und Einzelaktivitäten (Transkript 15), mehreren Einzelaktivitäten (Transkript 16) oder multiplen Ensembleaktivitäten (Transkript 17) bestehen. Je nach Verhältnis von Einzel- und Ensembleaktivität variieren dabei die multimodalen Ausformungen einerseits und die koordinativen Praktiken andererseits.

\subsubsection{Singuläre Beteiligungsmarker der simultanen Involvierung in multiple Aktivitäten}

Wie die bisherigen Analysen gezeigt haben, stehen die Interagierenden in Ensembleaktivitäten konstant vor der Aufgabe einander ihren Teilnehmendenstatus (Goodwin 2000d; Goodwin \& Goodwin 2004; Goodwin 2007) an einer oder mehrerer Aktivitäten anzuzeigen. Mithilfe von Pausierungen können Interagierende zwar eine andauernde Relevanz einer Aktivität aufrechterhalten, die tatsächliche Bearbeitung dieser Relevanz erfolgt dabei allerdings nicht, da die jeweilige Beteiligungsweise für die Dauer der Pausierung eingefroren wird. Um daher die gleichzeitige Relevanz mehrerer Aktivitäten einlösen zu können, greifen Interagierende auf Verfahren der Beteiligungsmarkierung zurück, mit denen sie zwei (oder mehr) Aktivitäten gleichzeitig bearbeiten können. In den folgenden drei Transkripten 12-14 leisten Interagierende eine solche Beteiligungsmarkierung, indem sie für die Herstellung der engagement displays nur eine singuläre Beteiligungsweise verwenden. Diese können neben verbal abstinenten engagement dis- 
plays (Transkript 12) auch verbale Marker umfassen. Wie sich im direkten Vergleich zwischen einer dyadischen (Transkript 13) und einer triadischen Interaktionskonstellation (Transkript 14) zeigen wird, kann eine Partikel als singulärer Involvierungsmarker zwar eine Simultanbearbeitung zweier Aktivitäten ermöglichen, geht aber mit interaktionalen Implikationen für das Interaktionsensemble einher. Dies lässt sich deutlich anhand der Beteiligungsweisen der Ko-Interagierenden nachweisen, wenn diese aufgrund des Involvierungsmarkers zwar ein engagement display bzgl. der gemeinsamen Aktivität interpretieren, ihre Interaktionspartner*innen aber dennoch danach auswählen, inwiefern diese ihnen ein visuelles Aufmerksamkeitsdisplay anbieten. Dass ein solches visuelles Aufmerksamkeitsdisplay von den Beteiligten aktiv eingefordert wird (5.2.2: Transkript 10) bzw. dessen Ausbleiben zum Abbruch der Ensembleaktivität führen kann (5.1.1: Transkript 2), ist bereits deutlich geworden. Solche visuellen Aufmerksamkeitsdisplays ermöglichen es den Teilnehmenden, simultane engagement displays für multiple Aktivitäten zu realisieren. In den folgenden Transkripten beteiligt sich jeweils ein ${ }^{\star}$ e Interagierende* ${ }^{\star}$ verbal an einer Ensembleaktivität und verfügt epistemische Autorität bzgl. des Gesagten, während eine andere Person sowohl Involvierung in eine Ensemble- als auch in eine Einzelaktivität anzeigt.

Tab. 16: Nicken bzw. Blickkontakt als singuläre Beteiligungsmarker an der Ensembleaktivität bei simultaner Involvierung in die Einzelaktivität durch SCM.

\begin{tabular}{|c|c|c|c|}
\hline Transkript 12 & a) & b) & c) \\
\hline Einzelaktivität: & engagement display & engagement display & engagement display \\
\hline \multirow[t]{3}{*}{ AUSPACKEN } & - - & - --1-- & - \\
\hline & haptische & haptische & haptische $\&$ visuelle \\
\hline & Beteiligungsweise & Beteiligungsweise & Beteiligungsweisen \\
\hline Ensembleaktivität: & Blick \& Nicken als & Blickkontakt als & disengagement display \\
\hline \multirow[t]{2}{*}{ ERKLÄREN } & Beteiligungsmarker & Beteiligungsmarker & $\begin{array}{l}\text { Aktivitätsabschluss } \\
\text { durch HOM }\end{array}$ \\
\hline & 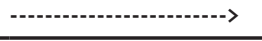 & - & --> \\
\hline
\end{tabular}

Damit wird es für den Schauspieler (SCM) im folgenden Transkriptausschnitt ${ }^{43}$ möglich, dem verbal beteiligten Hospitanten (HOM) im Rahmen der gemeinsamen Ensembleaktivität ERKLÄREN durch Nicken und Blickkontakt seine Beteiligung anzuzeigen, während er gleichzeitig seinen Rucksack AUSPACKT. Im Verlauf der Sequenz reduziert der Schauspieler sukzessive seine Beteiligungsweisen an der Ensembleaktivität von einem engagement display mit zwei Beteiligungsmar-

43 Es handelt sich hier um die feingraduellere Version des Transkripts 2. 
kern (Nicken und Blickkontakt) zu einer Partizipationsanzeige mit nur einem Beteiligungsmarker (Blickkontakt) bis er schließlich auch die visuelle Beteiligungsweise zugunsten seiner für ihn relevant gewordenen Einzelaktivität LESEN aufgibt, was der Hospitant als disengagement display bzgl. der Ensembleaktivität behandelt und die Ensembleaktivität daraufhin beendet (Tab. 16). Die Sequenz ist deshalb interessant, weil sie illustriert, inwiefern Blickkontakt als singulärer Beteiligungsmarker im Rahmen einer visuellen Beteiligungsweise für die Teilnehmenden ausreichen kann, damit sie ein engagement display bzgl. einer Ensembleaktivität annehmen.

\section{Transkript 12: Beteiligungsmarkierung an der Ensembleaktivität ERKLÄREN via} Nicken \& Blickkontakt während simultaner Realisierung der Einzelaktivität AUSPACKEN

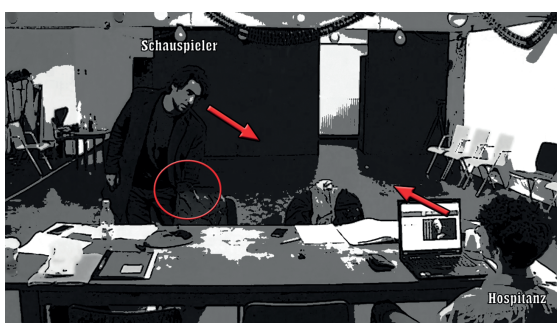

Abb. 12.1: SCM nickt beim ENTPACKEN.

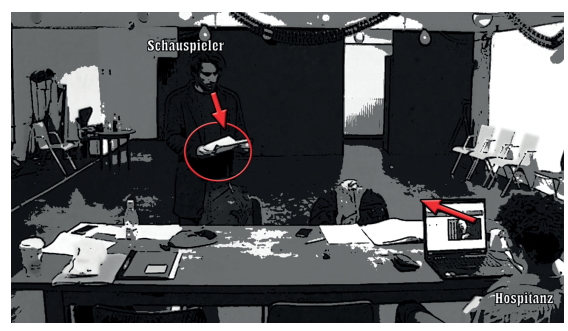

Abb. 12.2: SCM LIEST im Text, HOM BERICHTET weiter.

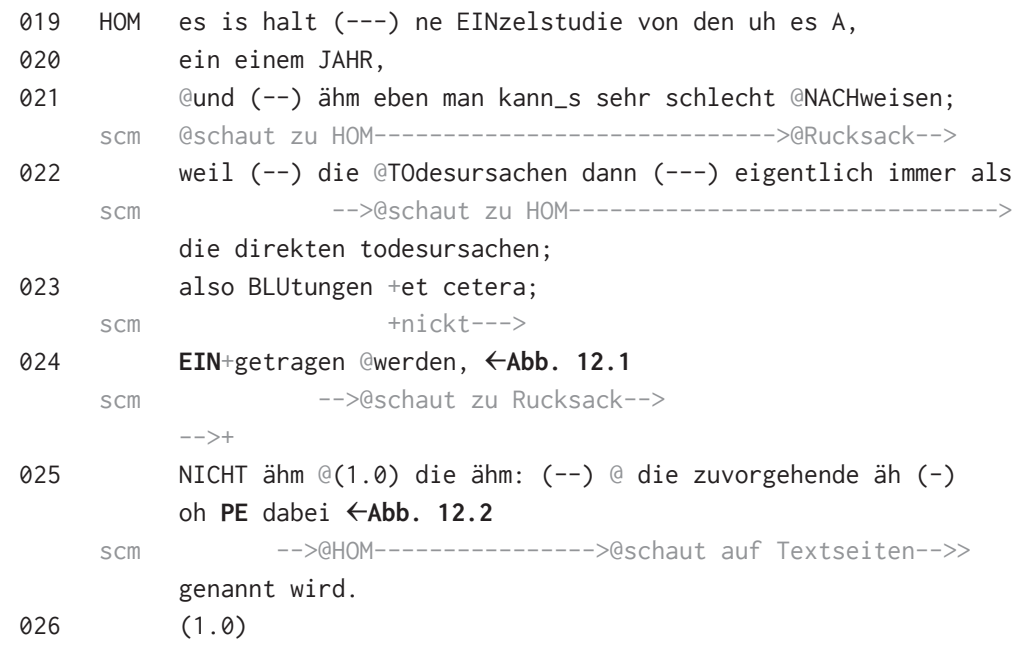


a) Blickkontakt und Nicken als Beteiligungsmarker bzgl. der Ensembleaktivität bei gleichzeitigem engagement display bzgl. der Einzelaktivität via haptischer Beteiligungsweise (Z.019-24): Während der Hospitant seine ERKLÄRUNG bzgl. einer Studie zu Todesursachen bei medizinischen Operationen durch verbale Interaktionsbeiträge ausführt und dabei visuell durchgängig auf den Schauspieler orientiert ist (Z.019-25), alterniert der Schauspieler konstant zwischen einer visuellen Orientierung auf seinen Rucksack und auf den Hospitanten. Auffällig ist dabei die Dauer der jeweiligen Blicke: Die Zeit, die der Schauspieler auf seinen Rucksack schaut, ist mit insgesamt 3.75 Sekunden deutlich kürzer als jene, die Blickkontakt mit dem Hospitanten umfasst (insgesamt 13.57 Sekunden). Damit zeigt der Schauspieler seine Orientierung auf die Ensembleaktivität an, indem er seine simultan relevante Einzelaktivität so organisiert, dass er ein möglichst umfangreiches, visuelles engagement display an der Ensembleaktivität anzeigen kann. Darüber hinaus bettet der Schauspieler in dieses Display ein Nicken ein, das es ihm ermöglicht, verbal abstinent ein bestätigendes Rezipientensignal (Goodwin 1980b; Goodwin 1984; Heath 1986) zu produzieren, während er gleichzeitig im Rahmen seiner haptischen Beteiligungsweise seiner Einzelaktivität AusPACKEN tief in seinen Rucksack greift (Z.023-4; Abb. 12.1).

b) Blickkontakt als Beteiligungsmarker bzgl. der Ensembleaktivität bei gleichzeitigem engagement display bzgl. der Einzelaktivität via haptischer Beteiligungsweise (Z.025): Obwohl der Schauspieler keine verbale Beteiligung an der Ensembleaktivität produziert, interpretiert der Hospitant diese sprachfreien go-aheads (Zinken \& Deppermann 2017) als Beteiligungsmarker des Schauspielers an der Ensembleaktivität. Dies ist auch dann der Fall, als der Schauspieler sein Nicken als gestische Beteiligungsweise einstellt und sein engagement display an der Ensembleaktivität nur noch über seine visuelle Beteiligungsweise anzeigt. In der Reaktion des Hospitanten, der in seiner verbalen und visuellen Beteiligungsweise unverändert fortfährt, wird deutlich, dass für ihn dieser singuläre Beteiligungsmarker des Schauspielers in Form des Blickkontakts ausreicht, damit er den Schauspieler als Teilnehmer der Aktivität annehmen kann.

c) disengagement display bzgl. der Ensembleaktivität bei gleichzeitigem engagement display bzgl. der Einzelaktivität via haptischer und visueller Beteiligungsweisen (Z.025-026): Die Annahme des Hospitanten hinsichtlich der Beteiligung des Schauspielers an der Ensembleaktivität verändert sich, als der Schauspieler für fast drei Sekunden keine Beteiligungsweisen bzgl. der Ensembleaktivität produziert und stattdessen seine Einzelaktivität via haptischer und visueller Beteiligungsweisen bearbeitet (Z.025, Abb. 12.2). Der Hospitant behandelt dieses Verhalten des Schauspielers als disengagement display bzgl. der Ensembleaktivität 
und führt seine damit die nun als Einzelaktivität fortgeführte Aktivität zu einem syntaktisch und prosodisch markierten Abschluss (Z.025).

Diese Sequenz liefert einen Hinweis auf jene Beteiligungsweisen, die vorhanden sein müssen, damit Interagierende ein engagement display annehmen können. Sowohl die Kombination aus Nicken und Blickkontakt als auch der Blickkontakt allein reichen hier aus, um als go-ahead fungieren zu können und damit die Ensembleaktivität aufrechtzuerhalten. Sobald der Blickkontakt jedoch fehlt, tritt das ein, was auch Goodwin (1980a) beschreibt: Der Sprechende pausiert bei fehlendem Blickkontakt des Hörenden in seiner Sprachproduktion. Für das vorliegende Transkript bedeutet das, dass die bloße Ko-Präsenz des Schauspielers für den Hospitanten nicht ausreicht, um den Schauspieler als Teil des Interaktionsensembles anzunehmen und die Aktivität fortzuführen. Stattdessen schließt der Hospitant die Aktivität aufgrund des disengagement displays des Schauspielers ab. Damit zeigt der Fall, welche zentrale Rolle der Blickkontakt nicht nur als Ressource zum Organisieren sprachlicher Beteiligungsweisen, sondern auch als Beteiligungsmarker bei der simultanen Koordination multipler Aktivitäten spielen kann.

Damit nicht jeder fehlende Blickkontakt in Situationen der simultanen Bearbeitung multipler Aktivitäten zu Aktivitätsabbrüchen führt, können Interagierende unter anderem auf Involvierungsmarker in Form von Rückmeldepartikeln (vgl. Schwitalla 2002: 266-271) zurückgreifen, die sie zeitlich abgestimmt dann realisieren, wenn sie den Blickkontakt abbrechen. Deutlich wird dies an folgendem Transkriptausschnitt, der einsetzt, nachdem der Regisseur auf einen Vorschlag durch die Schauspielerin mit einem GEGENVORSCHLAG geantwortet hat und dafür seine Einzelaktivität TASCHE AUSPACKEN pausiert hat ${ }^{44}$.

Um diese Einzelaktivität nach seinem Turnende zum Abschluss zu bringen, muss der Regisseur das AUSZUPACKENDE Etui nicht nur aus der Tasche herausholen, sondern es auch auf einen neuen Platz befördern. Dazu muss er zunächst einen geeigneten Platz auswählen. Dies erfolgt wie schon bei der Organisation der Einzelaktivität AUSPACKEN in Transkript 12 nicht allein über haptische Praktiken, sondern erfordert den Einsatz des Blicks. Das bedeutet allerdings, dass der Regisseur den Blickkontakt als Beteiligungsweise an der Ensembleaktivität mit der Schauspielerin abbrechen muss. Der Regisseur bearbeitet dieses Problem innerhalb der gleichzeitigen Relevanz multipler Aktivitäten, indem er die Partikel „hmhm“ (Z.019) in dem Moment äußert, in dem er seinen Blick verschiebt. Die Partikel

44 Transkript 13 schließt damit unmittelbar an Transkript 6 an, in dem der Regisseur seine Einzelaktivität AUSPACKEN pausiert, um der Schauspielerin auf ihren VoRSCHLAG einen GEGENVORscHLAG anzubieten. 
Tab. 17: hmhm als singulärer Beteiligungsmarker an der Ensembleaktivität bei simultaner Involvierung in die Einzelaktivität durch REG.

\begin{tabular}{|c|c|c|c|}
\hline Transkript 13 & a) & b) & c) \\
\hline Einzelaktivität: & engagement display & engagement display & engagement display \\
\hline \multirow[t]{3}{*}{ AUSPACKEN } & 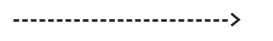 & 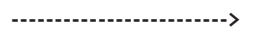 & 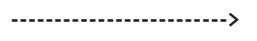 \\
\hline & haptische & haptische \& visuelle & haptische \\
\hline & Beteiligungsweise & Beteiligungsweisen & Beteiligungsweise \\
\hline Ensembleaktivität: & engagement display & Partikel als & engagement display \\
\hline \multirow[t]{4}{*}{ VORSCHLAGEN } & & Beteiligungsmarker & restart durch SCW \\
\hline & 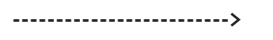 & 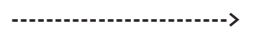 & -----------> \\
\hline & visuelle & verbale & visuelle \\
\hline & Beteiligungsweise & Beteiligungsweise & Beteiligungsweise \\
\hline
\end{tabular}

zeigt der Schauspielerin an, dass der Regisseur trotz fehlenden Blickkontakts als Beteiligungsmarkierung (vgl. Transkript 12) an der Ensembleaktivität partizipiert. Deutlich wird diese Organisation der Beteiligungsmarkierung vor allem mit Blick auf die Schauspielerin, die ihre verbale Beteiligungsweise auch ohne Blickkontakt mit ihrem Adressaten fortsetzt (Tab. 17). Damit erfüllt die Partikel hmhm hier die Funktion einer Beteiligungsmarkierung zur simultanen Bearbeitung gleichzeitig relevanter Aktivitäten, indem sie den Abzug der visuellen Beteiligungsweise kompensier $\mathrm{t}^{45}$. Dass dies nur teilweise gelingt, zeigt das folgende Transkript.

\section{Transkript 13: Beteiligungsmarkierung an der dyadischen Ensembleaktivität VORSCHLAGEN via Rückmeldepartikel während simultaner Realisierung der Einzelaktivität AUSPACKEN}

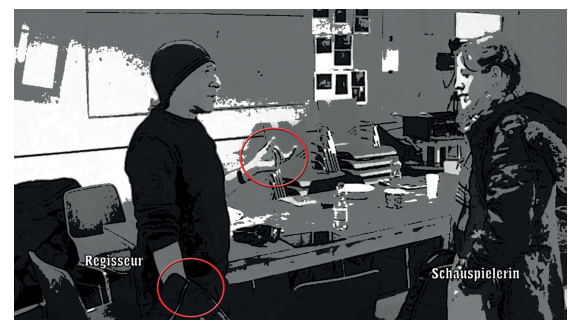

Abb. 13.1: REG behält Körperkonfiguration bei.

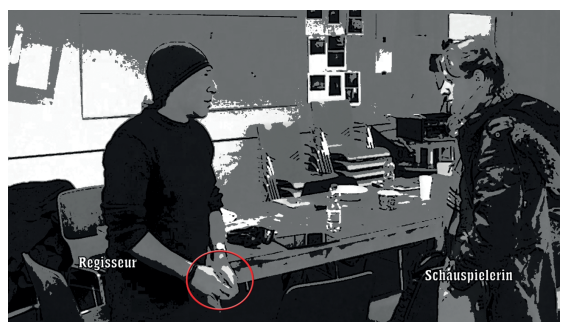

Abb. 13.2: REG fasst sein Brillenetui mit beiden Händen.

45 Auf die Verfahren der Kompensation (Routinisierung und Synchronisierung), auf die Interagierende zurückgreifen können, um auch strukturell inkompatible Aktivitäten simultan zu koordinieren, wird in Kapitel 6 und besonders in Kapitel 7 eingegangen. 


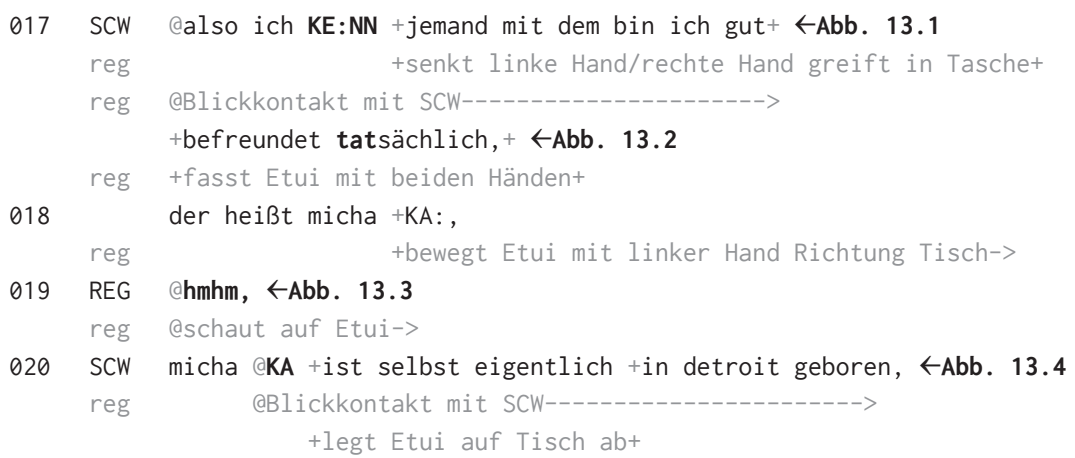

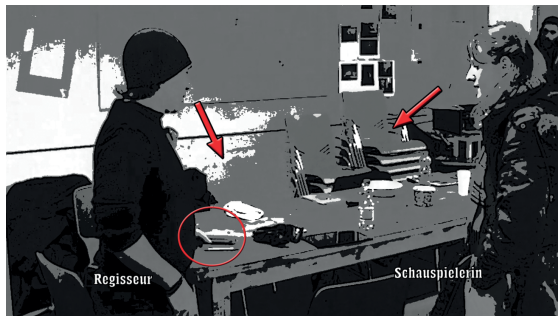

Abb. 13.3: REG und SCW verfolgen das Etui.

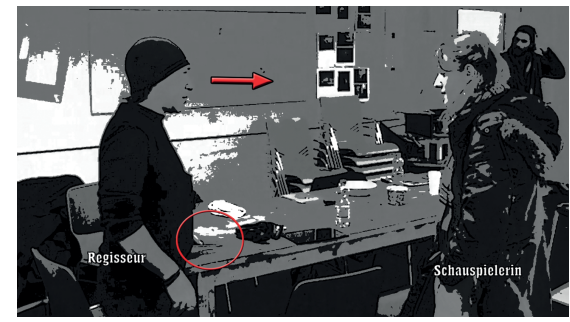

Abb. 13.4: REG stellt wieder Blickkontakt her.

a) engagement display bzgl. der Ensembleaktivität via visueller Beteiligungsweise bei gleichzeitigem engagement display bzgl. der Einzelaktivität via haptischer Beteiligungsweise (Z.017-018): Der Regisseur richtet sein multimodales Verhalten auf die Bearbeitung der gleichzeitigen Relevanz der Ensembleaktivität VoRSCHLAGEN und der Einzelaktivität AUSPACKEN aus, indem er seine Beteiligungsweisen auf beide Aktivitäten aufteilt. Mithilfe der visuellen Beteiligungsweise hält er Blickkontakt mit der verbal beteiligten Schauspielerin, während er seine Hände so organisiert, dass er gleichzeitig im Rahmen der haptischen Beteiligungsweise das Etui mit der rechten Hand aus der Tasche herausholt und es in die linke Hand überführt (Z.017, Abb. 13.1/2). Dadurch ist er in der Lage, das Etui mit der linken Hand in Richtung des Tisches, der als Ablageort des Objekts fungiert, zu bewegen und der Schauspielerin gleichzeitig mithilfe des Blickkontakts sein engagement display an der Ensembleaktivität anzuzeigen (Z.018). Diesen interaktionalen Spagat zwischen den beiden Aktivitäten hält er aufrecht, bis seine Einzelaktivität ebenfalls eine visuelle Beteiligung erfordert.

b) Partikel als Beteiligungsmarker bzgl. der Ensembleaktivität bei gleichzeitigem engagement display bzgl. der Einzelaktivität via haptischer und visueller Beteili- 
gungsweisen (Z.019): Um zu überprüfen, wohin er das Etui auf den Tisch legt, bricht der Regisseur den Blickkontakt mit der Schauspielerin ab. Dies vollzieht er in dem Moment, in dem die Schauspielerin im Rahmen ihres VoRschlags einen potentiell relevanten Musiker („micha KA“, Z.018) namentlich einführt und damit ihre Aussage, sie würde diesen Musiker gut kennen, faktisch unterstützt (vgl. Kretzenbacher 2010). Diese Information ratifiziert der Regisseur mit einer reduplizierten Rückmeldepartikel mit fallend-steigender prosodischen Kontur „hmhm“ (Z.019) (vgl. Ehlich 1986: 54; Nübling 2005), während er simultan den Blick zum Tisch verändert und den linken Arm mit dem Brillenetui in Richtung des Tisches bewegt. Die Schauspielerin folgt dem Blick des Regisseurs zum Brillenetui und damit der Trajektorie seiner Einzelaktivität (Abb. 13.3). Die Partikel „hmhm“ (Z.019) ermöglicht an dieser Stelle eine Koordinierung der gleichzeitig relevanten Aktivitäten, mit der der Regisseur weiterhin ein engagement display bzgl. der Ensembleaktivität anzeigen kann, während er die Einzelaktivität AusPACKEN realisiert. Wie eng der Moment der visuellen Reorientierung mit der Partikel verbunden ist, zeigt sich darin, wie die Beteiligten sich gemeinsam auf die Ensembleaktivität ausrichten.

c) engagement display bzgl. der Ensembleaktivität via visueller Beteiligungsweise bei gleichzeitigem engagement display bzgl. der Einzelaktivität via haptischer Beteiligungsweise (Z.020): Der Regisseur stellt sein visuelles engagement display bzgl. der Ensembleaktivität wieder her, noch bevor seine Einzelaktivität abgeschlossen ist (Z.020). Während er das Brillenetui auf den Tisch legt und damit seine AusPACK-Aktivität in deren Endphase überführt, orientiert er sich visuell wieder zurück auf die Schauspielerin (Abb. 13.4). Diese erwidert den Blickkontakt und nimmt das letzte Sprachmaterial aus ihrer vorherigen Äußerung in Form eines „restarts“ (vgl. Goodwin 1980a) wieder auf („micha KA“, Z.020). Für den Restart recycelt die Schauspielerin zwar zunächst den Namen des Musikers, dessen prosodische Kontur sich aber in Hinblick auf die erste Nennung (Z.018) dahingehend unterscheidet, dass bei der zweiten Nennung die steigende Intonation fehlt. Darüber hinaus erweitert sie beim Restart die Äußerung inkrementell um eine weitere Information bzgl. des Geburtsortes des Musikers, die vor dem Restart noch nicht genannt wurde. Betrachtet man das Timing des Restarts im multimodalen Vollzug, fällt auf, dass die Schauspielerin in ihrer verbalen Beteiligung an der Ensembleaktivität fortfährt, noch bevor der Regisseur sie wieder anschaut (Z.020). Damit reagiert die Schauspielerin in dem Moment auf eine Veränderung im Gesprächsfluss (=Ausbleiben des Blickkontakts ihres Interaktionspartners), in dem der Regisseur eine Rückmeldepartikel realisiert. In ihrem Äußerungsdesign des Restarts wird deutlich, dass sie die Ensembleaktivität als von beiden Interagierenden weiterhin als relevant angesehen behandelt. 
Die Rückmeldepartikel als Beteiligung an der Ensembleaktivität im Moment des von beiden Interagierenden wahrgenommen Wechsels der visuellen Orientierung auf das Ziel der Einzelaktivität leistet an dieser Stelle eine Beteiligungsmarkierung bzgl. der Ensembleaktivität, die ohne diese Involvierungsanzeige seitens des Regisseurs ohne engagement display gewesen wäre. Mithilfe der Partikel hält der Regisseur seine Partizipation an der Ensembleaktivität aufrecht, während er eine Einzelaktivität bearbeitet. Gleichzeitig liefert sie der Schauspielerin ein Signal dafür, dass der Regisseur ihre Ausführungen weiterhin (auditiv) monitort. Somit erfüllt die Partikel hmhm hier mehr als nur die „kontinuierliche[n] Rückmeldungen über die mentalen Prozesse der Verarbeitung des Gesprochenen“ (Ehlich 2009: 430) und geht über eine verbale Markierung von Abbrüchen oder Wiederaufnahmen hinaus, wie die Verwendung von Partikeln in der Forschungsliteratur im Zusammenhang mit multiplen Aktivitäten bisher beschrieben worden ist (Yang, Heeman \& Kun 2011; Sutinen 2014). Die Verwendung von $\mathrm{hmhm}$ als Beteiligungsmarker leistet in dieser Sequenz somit kein entweder oder, sondern ein sowohl als auch der multiplen Aktivitäten als Konsequenz der simultanen Bearbeitung gleichzeitiger Relevanz.

Obwohl die visuelle Antizipation der Einzelaktivität des Regisseurs durch die Schauspielerin, ihre beibehaltene Körperorientierung auf ihn und ihre veränderte prosodische Kontur ihre Interpretation des aufrechterhaltenen Status der Ensembleaktivität anzeigt, liefert ihr Äußerungsdesign mit ihrem sprachmaterialrecycelnden Restart (Z.020) nach Verwendung des Beteiligungsmarkers nichtsdestotrotz einen Hinweis darauf, dass sie eine Veränderung im Interaktionsfluss bearbeitet. Die Vermutung, dass sie hier nicht nur auf die Unterbrechung durch die Partikel reagiert, sondern den Restart vorrangig als Antwort auf den fehlenden Blickkontakt ihres Interaktionspartners konzipiert, bestärkt sich im direkten Vergleich mit dem folgenden Transkript. Darin greift der Schauspieler (SCM) auf die Rückmeldepartikel ja zurück, um seine Beteiligung an der Ensembleaktivität BERICHTEN anzuzeigen, während er sich visuell auf seine Einzelaktivität AUSPACKEN orientiert. Obwohl der verbal beteiligte Regisseur (REG) in seinem Äußerungsdesign anzeigt, dass er den Schauspieler weiterhin als Mitglied der Ensembleaktivität versteht, wechselt er in seinem Folgeturn seine visuelle Aufmerksamkeit auf die Schauspielerin (SCW) als Ko-Teilnehmerin der triadischen Ensembleaktivität.

Die Partikel als singulärer Beteiligungsmarker wird in folgendem Transkript 14 aus dem gleichen Grund notwendig, wie bereits in Transkript 13. Die Einzelaktivität AUSPACKEN, die vom Schauspieler vor allem mithilfe haptischer Beteiligungsweisen vollzogen wird, benötigt zusätzlich den Einsatz einer visuellen Beteiligungsweise, die der Schauspieler allerdings in der laufenden Interaktion dafür verwendet, ein engagement display bzgl. der Ensembleaktivität BERICHTEN 
Tab. 18: $j a$ als singulärer Beteiligungsmarker an der Ensembleaktivität bei simultaner Involvierung in die Einzelaktivität durch SCM.

\begin{tabular}{|c|c|c|c|}
\hline Transkript 14 & a) & b) & c) \\
\hline Einzelaktivität: & engagement display & engagement display & engagement display \\
\hline \multirow[t]{3}{*}{ AUSPACKEN } & 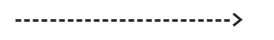 & 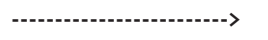 & 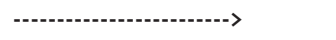 \\
\hline & haptische & visuelle $\&$ haptische & haptische \\
\hline & Beteiligungsweise & Beteiligungsweisen & Beteiligungsweise \\
\hline Ensembleaktivität: & engagement display & Partikel als & engagement display \\
\hline \multirow[t]{4}{*}{ BERICHTEN } & & Beteiligungsmarker & Wechsel im \\
\hline & & & Adressatenzuschnitt bei REG \\
\hline & visuelle & verbale & visuelle \\
\hline & Beteiligungsweise & Beteiligungsweise & Beteiligungsweise \\
\hline
\end{tabular}

anzuzeigen. Der zeitlich abgestimmte Einsatz der Partikel als verbaler Beteiligungsweise an der Ensembleaktivität ermöglicht es dem Schauspieler also zu verhindern, seinen Ko-Interagierenden ein disengagement display bzgl. der Ensembleaktivität anzubieten und zeitgleich seine Einzelaktivität zu bearbeiten (Tab. 18).

\section{Transkript 14: Beteiligungsmarkierung an der triadischen Ensembleaktivität BERICHTEN via Rückmeldepartikel während simultaner Realisierung der Einzelaktivität AUSPACKEN}

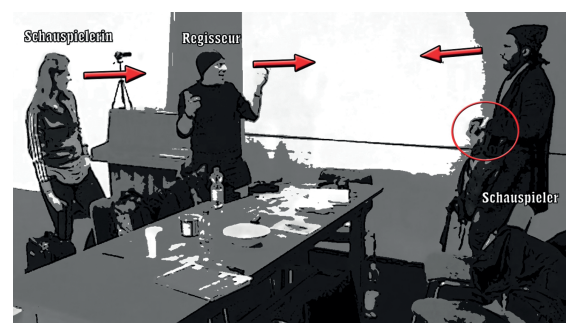

Abb. 14.1: REG orientiert sich auf SCM.

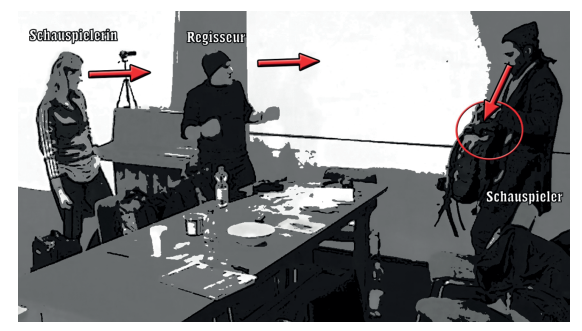

Abb. 14.2: SCM schaut auf Rucksack.

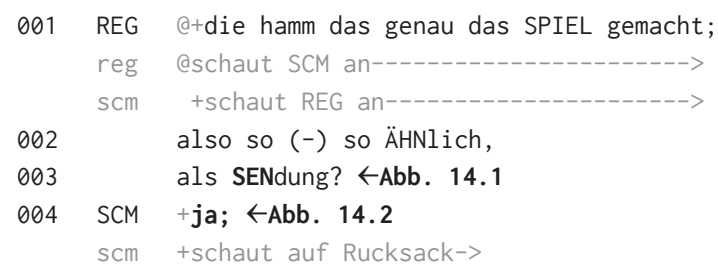




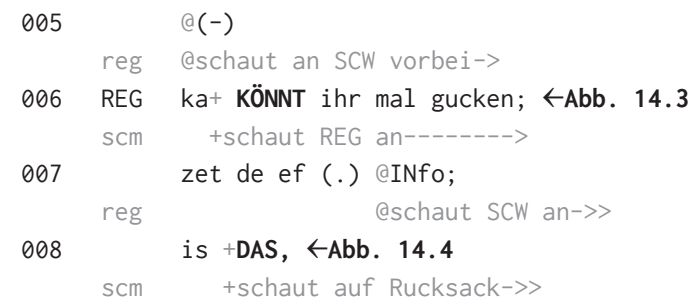

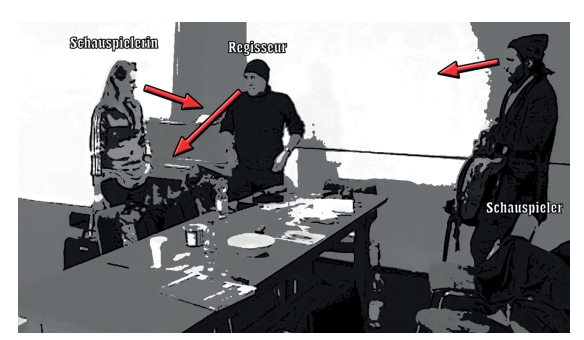

Abb. 14.3: SCM greift in Rucksack.

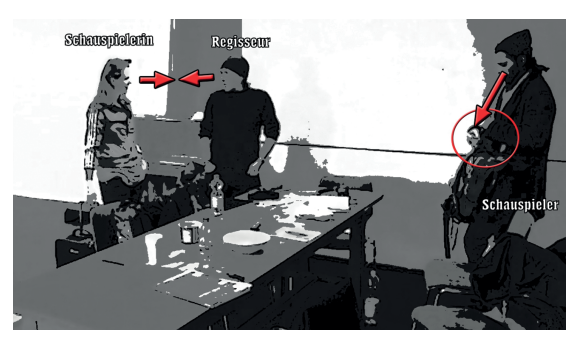

Abb. 14.4: REG orientiert sich auf SCW.

a) engagement display bzgl. der Ensembleaktivität via visueller Beteiligungsweise bei gleichzeitigem engagement display bzgl. der Einzelaktivität via haptischer Beteiligungsweise (Z.001-003): In dieser Sequenz BERICHTET der Regisseur von einer Fernsehsendung, in der das Publikum über den Ausgang einer für die betreffende Narration zentrale Entscheidung bestimmen konnte, so wie es auch für dieses Theaterstück geplant ist. Er orientiert sich im Verlauf des BERICHTS (Z.001-3) visuell zum Schauspieler, der den Blickkontakt erwidert und damit per visueller Beteiligungsweise sein engagement display bzgl. der Ensembleaktivität herstellt. Parallel dazu öffnet der Schauspieler seinen Rucksack und ist damit in die Startphase seiner AuSPACK-Einzelaktivität involviert (Abb. 14.1). Wie bereits der Regisseur in Transkript 13 organisiert der Schauspieler hier seine multimodalen Ressourcen dergestalt, dass er beide Aktivitäten simultan bearbeiten kann. Mithilfe des Blickkontakts stellt er dem Regisseur ein engagement display bzgl. der Ensembleaktivität zur Verfügung, während er mit haptischer Beteiligungsweise seine Einzelaktivität vollzieht. Diese simultane Koordinierung hält er solange aufrecht, bis seine Einzelaktivität ebenfalls eine visuelle Ressource benötigt.

b) Partikel als Beteiligungsmarker bzgl. der Ensembleaktivität bei gleichzeitigem engagement display bzgl. der Einzelaktivität via haptischer und visueller Beteiligungsweisen (Z.04-005): In Transkript 13 ist es das Lokalisieren eines geeigneten Ablageplatzes für das Etui des Regisseurs, hier lokalisiert der Schauspieler mithilfe der multimodalen Ressource Blick den Gegenstand, den er AUSPACKEN wird. 
In beiden Transkripten realisieren die Auspackenden eine Rückmeldepartikel am TRP des verbalen Beitrags, während sie den Blickkontakt und damit die visuelle Beteiligung an der Ensembleaktivität abbrechen. Dass es zu keiner verbalen Überlappung kommt, zeigt eine Orientierung der Auspackenden an der jeweils laufenden Ensembleaktivität. Sie passen den Einsatz der Partikeln zeitlich so ab, dass sie nicht mit einer anderen Äußerung überlappen und halten ihre Einzelaktivitäten solange zurück, bis der sequentielle Slot innerhalb der Ensembleaktivitäten dafür bereitsteht. Die Partikel „ja“ (Z.004) ermöglicht dem Schauspieler in dieser Sequenz eine präzise getimte Beteiligung an der Ensembleaktivität zum Zeitpunkt der Veränderung seines visuellen engagement displays. Damit leistet die Partikel zwar eine Bestätigung des Gesagten, geht aber in ihrer Funktion „retraktiv eine eröffnete Leerstelle zu füllen“ (Meer 2009: 95) hinaus, indem sie als singulärer Beteiligungsmarker innerhalb der multimodalen Gestalt fungiert.

c) engagement display bzgl. der Ensembleaktivität via visueller Beteiligungsweise bei gleichzeitigem engagement display bzgl. der Einzelaktivität via haptischer Beteiligungsweise (Z.006-008): Wie bereits in Transkript 13 hat auch hier das Ausbleiben des Blickkontakts interaktionale Auswirkungen auf die Emergenz der Sprachproduktion: Nachdem der Schauspieler seinen Blick abgewendet hat, pausiert der Regisseur zunächst in seiner verbalen Beteiligung (Z.005). Als er seinen Turn fortsetzt, schaut er nicht nur keine*n der Anwesenden an (Abb. 14.3), sondern zeigt auch in einer Selbstreparatur („ka KÖNNT ihr mal gucken“, Z.006) seine Interpretation der veränderten Teilnehmendenrahmung an. So bricht der Regisseur die Äußerung des Modalverbs können in der zweiten Person Singular (kannst) zugunsten der Konjugation in der zweiten Person Plural (könnt) ab. Damit macht er zweierlei in seinem Adressatenzuschnitt deutlich: Zum einen ist der Schauspieler nun nicht mehr der alleinige Adressat seines Redebeitrags. Dies zeigt sich auch im Wechsel der Körperorientierung des Regisseurs vom Schauspieler zur Schauspielerin, die den Blickkontakt mit dem Regisseur erwidert (Abb. 14.4). Zum anderen behandelt der Regisseur den Schauspieler weiterhin als ratifizierten Beteiligten (Goffman 1981) der Ensembleaktivität, bearbeitet aber das Ausbleiben des Blickkontakts durch den Adressaten (Goodwin 1980a) mit einem Orientierungswechsel.

In den Transkripten 12, 13 und 14 verfügt jeweils ein ${ }^{\star}$ e Interagierende ${ }^{\star}$ r epistemische Autorität bzgl. der verbalen Beteiligungsweise an einer Ensembleaktivität, während eine andere Person sowohl Involvierung in eine Ensemble- als auch in eine Einzelaktivität anzeigt. In allen drei Transkripten produzieren die zusätzlich in Einzelaktivitäten involvierten Beteiligten per Blickkontakt (und in Transkript 12 darüber hinaus per Nicken) engagement displays bzgl. der Ensembleaktivität. 
Um die Einzelaktivitäten fokussieren zu können, brechen die Interagierenden in allen besprochenen Fällen den Blickkontakt mit den sprechenden Interagierenden ab. Daraufhin bearbeiten die verbal Beteiligten den fehlenden Blickkontakt, indem sie auf die veränderten Interaktionsbedingungen reagieren: Während in den Transkripten 13 und 14 diejenigen, die den Blickkontakt auflösen, im Moment der visuellen Reorientierung Rückmeldepartikeln ( $\mathrm{hmhm}$ bzw. ja) produzieren, bleibt eine solche Beteiligungsmarkierung durch den blickkontaktabbrechenden Interagierenden in Transkript 12 aus. Als Resultat daraus behandelt der sprechende Interagierende den Ko-Interagierenden in Transkript 12 als nicht mehr in die Ensembleaktivität involviert und beendet die Ensembleaktivität. Im Gegensatz dazu bleibt die Ensembleaktivität nach der Realisierung der Rückmeldepartikeln in den Transkripten 13 und 14 bestehen. Die verbal beteiligten Interagierenden bearbeiten dort den fehlenden Blickkontakt per Restart (Transkript 13) oder Selbstreparatur mit Veränderung in der Teilnehmendenrahmung (Transkript 14). Sie reagieren damit auf eine Veränderung in der Interaktionskonfiguration, behandeln ihre Ko-Interagierenden (im Gegensatz zu Transkript 12) aber weiterhin als ratifizierte Teilnehmende des Interaktionsensembles. Damit wird deutlich, dass die Rückmeldepartikeln hmhm und ja in den Transkripten 13 und 14 als singuläre Beteiligungsmarker verwendetet werden, um mit der gleichzeitigen Relevanz multipler Aktivitäten umzugehen, wenn aufgrund eines foregroundings (Langacker 1987, 2008) der Einzelaktivität kein visuelles engagement display bzgl. der Ensembleaktivität via Blickkontakt angezeigt werden kann, aber beide Aktivitäten gleichermaßen bearbeitet werden.

\subsubsection{Multiple Beteiligungsmarker der simultanen Involvierung in multiple Aktivitäten}

Zwei oder mehr gleichzeitig relevante Aktivitäten zur selben Zeit zu bearbeiten, stellt an Interagierende ein gesteigertes Maß inter- und intrapersoneller Koordinierungsanforderungen. Dies ist insbesondere dann der Fall, wenn nicht nur eine Involvierung in einer der Aktivitäten mithilfe eines singulären Beteiligungsmarkers (z. B. Nicken oder Rückmeldepartikel) angezeigt werden soll, sondern wenn die Beteiligungsweisen gleichmäßig auf die Aktivitäten verteilt werden, um die ko-relevanten Aktivitäten gleichermaßen bearbeiten zu können. Da die körperlichen Ressourcen des Menschen anatomisch determiniert sind (Menschen haben maximal zwei Hände, können mit beiden Augen in nur eine Richtung gleichzeitig schauen, usw.) erfordert dieses Verteilen der Beteiligungsweisen auf die Aktivitäten mitunter eine enge Verzahnung der Ressourcen im multimodalen Haushalt. Die Möglichkeiten, die multimodalen Ressourcen auf zwei gleichzeitig relevante 
Aktivitäten zur Bearbeitung derselben aufteilen zu können, variieren dabei je nach Kombination der Aktivitätsformen. Die simultane Koordinierung einer Einzel- mit einer Ensembleaktivität (Transkript 15) unterscheidet sich deutlich von der rein intrapersonellen Koordinierungsleistung zweier Einzelaktivitäten (Transkript 16) einerseits und der gesteigerten interpersonellen Koordinierungsleistung bei der simultanen Bearbeitung zweier gleichzeitig relevanter Ensembleaktivitäten (Transkript 17).

Tab. 19: Linke und rechte Hand als multiple Beteiligungsmarker an der Ensembleaktivität bei simultaner Involvierung in die Einzelaktivität durch SCW.

\begin{tabular}{|c|c|c|c|}
\hline Transkript 15 & a) & b) & c) \\
\hline $\begin{array}{l}\text { Einzelaktivität: } \\
\text { MANTEL AUSZIEHEN }\end{array}$ & & \begin{tabular}{l} 
engagement display \\
\hdashline linke Hand: \\
haptische \\
Beteiligungsweise
\end{tabular} & \\
\hline $\begin{array}{l}\text { Ensembleaktivität: } \\
\text { VoRSCHLAGEN }\end{array}$ & $\begin{array}{l}\text { engagement display } \\
\text { verbale \& visuelle } \\
\text { Beteiligungsweisen }\end{array}$ & $\begin{array}{l}\text { engagement display } \\
\text {---.---.--> } \\
\text { rechte Hand: } \\
\text { gestische } \\
\text { Beteiligungsweise } \\
\text { + verbale \& visuelle } \\
\text { Beteiligungsweisen }\end{array}$ & $\begin{array}{l}\text { engagement display } \\
\text { - beide Hände: gestische } \\
\text { Beteiligungsweise } \\
\text { + verbale \& visuelle } \\
\text { Beteiligungsweisen }\end{array}$ \\
\hline
\end{tabular}

Alle hier präsentierten Fälle zeigen die Orientierungen der Teilnehmenden auf die gleichzeitige Relevanz multipler Handlungsverläufe, die sie mit einem Aufteilen ihrer multimodal organisierten, strukturell kompatiblen Beteiligungsweisen bearbeiten. Dies erreicht die Schauspielerin im folgenden Transkriptausschnitt primär durch ihre Hände, mit denen sie sich einerseits gestisch an der VoRschlags-Ensembleaktivität mit dem Regisseur beteiligt, während sie im Rahmen ihrer Einzelaktivität MANTEL AUSZIEHEN ihren Reißverschluss nach unten zieht.

Das duale engagement display, im Rahmen dessen die Schauspielerin sowohl ihre Einzelaktivität bearbeitet als auch mithilfe verbaler und visueller Beteiligungsweisen an der Ensembleaktivität partizipiert, ist damit eine Praktik, bei der die Schauspielerin ihre Hände zeitgleich für unterschiedliche Aktivitäten verwendet. Während die linke Hand im Zuge einer haptischen Beteiligungsweise das MANTElauszieHen vollzieht, nutzt die Schauspielerin ihre rechte Hand als redebegleitende gestische Beteiligungsweise zur Konzeptvermittlung (Tab. 19). 


\section{Transkript 15: Simultane Beteiligung an der Ensembleaktivität VoRSCH LAGEN und die Einzelaktivität MANTEL AUSZIEHEN}

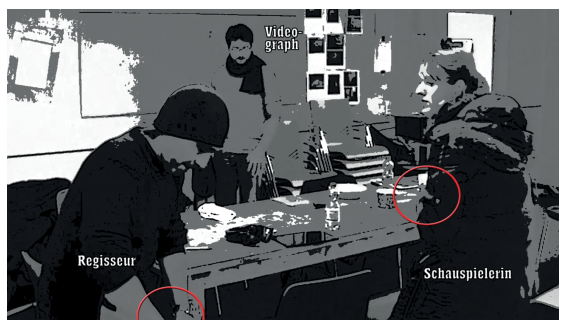

Abb. 15.1: SCW greift den Reißverschluss.

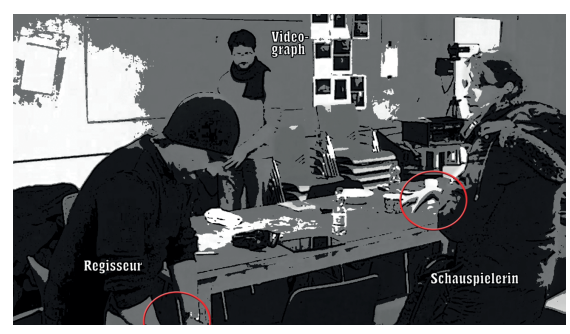

Abb. 15.2: SCW gestikuliert beim AuszieHEN.

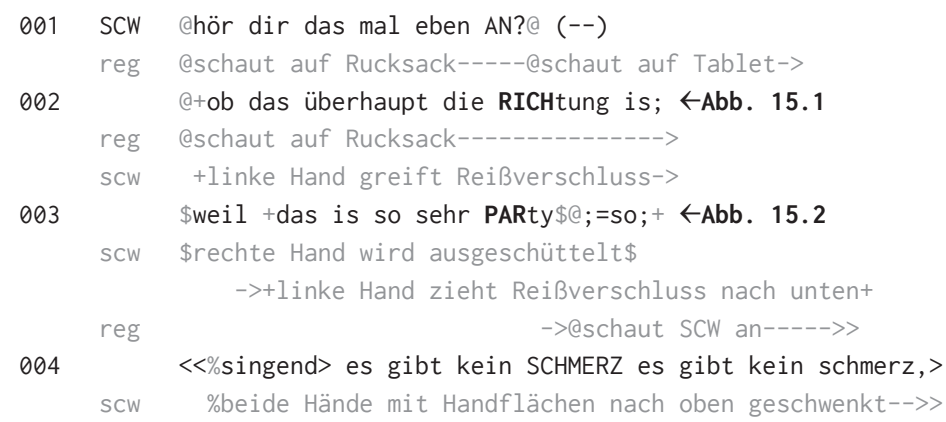

a) engagement display bzgl. der Ensembleaktivität via verbaler und visueller Beteiligungsweisen (Z.001-002): Die Schauspielerin monitort die Einzelaktivität AusPACKEN des Regisseurs (Abb. 15.1), den sie auffordert, ihren LIEDVORSCHLAG anzuhören (Z.001). Der Regisseur reagiert auf diese Aufforderung, indem er seinen Blick von seiner Tasche auf das Tablet verschiebt (Z.001), orientiert sich jedoch wieder auf seine Einzelaktivität, als die Schauspielerin kein Lied abspielt (Z.002). Die Schauspielerin reagiert auf diese Reorientierung des Regisseurs, indem sie ihre TCU inkrementell erweitert. Das Adverbialsatz-Inkrement „ob das überhaupt die RICHtung is“ (Z.002) ist dabei sowohl prosodisch (Sprechpause und veränderte Tonhöhenbewegung) von der Host-TCU abgegrenzt, als auch syntaktisch von ihr abhängig (vgl. Inkremente im Deutschen bei Auer 2007b) und fungiert als Einschränkung bzgl. ihres VoRschlaGs, den sie im Folgenden im Rahmen einer gesungen modulierten Konzeptvermittlung ausführt.

b) engagement display bzgl. der Ensembleaktivität via verbaler, visueller und gestischer Beteiligungsweise der rechten Hand bei gleichzeitigem engagement display 
bzgl. der Einzelaktivität via haptischer Beteiligungsweise der linken Hand (Z.003): Gleichzeitig tastet und fasst die Schauspielerin mit der linken Hand den Reißverschluss ihrer Jacke, ohne die visuelle Beteiligungsweise von der Ensembleaktivität abzuziehen. Als der Regisseur nach wie vor keine Orientierung bzgl. ihres VoRschlaGs anzeigt, macht die Schauspielerin bei der Beschreibung des Lieds (,so sehr PARty“, Z.003) „rhythmische“ Bewegungen mit ihrer rechten Hand, während ihre linke Hand simultan den Reißverschluss herunterzieht (Abb. 15.2). Beide Hände sind damit jeweils an einer anderen Aktivität beteiligt. Die linke Hand nutzt die Schauspielerin zur haptischen Beteiligung an der Einzelaktivität Auszienen. Im Gegensatz dazu bilden ihre Gestikulation, ihre Blick- und Körperorientierung auf den Regisseur sowie der Adressatenzuschnitt der den VORSCHLAG bereits im Vorfeld einschränkenden Beschreibung des Liedes eine komplexe Gestalt, mit der sie sich an der Ensembleaktivität VoRSCHLAGEN beteiligt. Die Schauspielerin vollzieht beide Aktivitäten simultan, wodurch die Multiaktivität AUSZIEHENDER-VORSCHLAG entsteht. Betrachtet man das koordinierende Timing, wird deutlich, dass die Beteiligungsweisen der Hände an den jeweiligen Aktivitäten einerseits zeitlich unabhängig voneinander sind und andererseits unterschiedliche Funktionen erfüllen. So ist der Einsatz der händischen Beteiligungsweisen eng mit der jeweiligen Aktivität verknüpft. Die Einzelaktivität MANTEL AUSZIEHEN kann simultan zur verbalen Beteiligungsweise an der Ensembleaktivität initiiert werden, von der sie strukturell und koordinativ nicht abhängig ist. Deutlich wird dies anhand des Zeitpunkts, an dem die Einzelaktivität bearbeitet wird. Denn während das Reißverschlussfassen noch im Übergang von einer Aufforderungshandlung zu einer Einschränkung vollzogen wird, zieht die Schauspielerin den Reißverschluss schließlich inmitten einer syntaktischen Konstruktion weil + Begründung (vgl. Z.003) herunter. Im Gegensatz dazu ist die händische Beteiligungsweise an der Ensembleaktivität in hohem Maße mit der verbalen Beteiligung verknüpft (vgl. Kendon 1980; Schegloff 1984; Streeck 1993): Die Schauspielerin beginnt erst mit den rhythmischen Bewegungen der rechten Hand, als die dazu passende semantische Kategorie Party eingeführt wird (vgl. McNeill 1992, 2005). In der Konsequenz werden Party-TCU und Party-Geste gemeinsam gestartet und gemeinsam abgeschlossen (vgl. Z.003).

c) Auflösen des dualen engagement displays zum engagement display bzgl. der Ensembleaktivität via verbaler, visueller und gestischer Beteiligungsweisen beider Hände (Z.003-004): Die Bearbeitung der Einzelaktivität ist indes noch nicht beendet und erfordert weiterhin, dass die Schauspielerin eine Hand am Mantel behält und den Reißverschluss nach unten zieht. Die Schauspielerin reagiert darauf, indem sie die Partikel „SO“ (Z.003) mit schnellem Anschluss realisiert. Dies verschafft ihr intrapersonell genügend Zeit, um den Reißverschluss vollstän- 
dig nach unten zu ziehen, die Einzelaktivität dadurch zu einem vorläufigen $\mathrm{Ab}$ schluss zu bringen und beide Hände zur Verfügung zu haben, um diese synchron zueinander als tanzende Bewegungsressource zur Beteiligung an ihrem gesungen modulierten LIEDVorschlag zu organisieren. Mit dem Ende der TCU der Schauspielerin (Z.003) fällt auf diese Weise das schwungvolle Herunterziehen des Reißverschlusses zusammen, sodass sie nach dem Inkrement „So“ (Z.003), das bereits die kommende Performance des Liedes projiziert (vgl. Auer 2006; Ningelgen \& Auer 2017), zum Beginn ihrer nächsten TCU (Z.004) beide Hände zum Gestikulieren frei hat. Sie erweitert daraufhin ihre Beschreibung des Liedes, indem sie das Lied singend und mit erhobenen Händen tanzend performt ${ }^{46}$ (Z.004). Damit zeigt sich zum einen die enge Verknüpfung der beiden Aktivitäten, wenn sich der noch offene Status der Einzelaktivität in Form einer Partikel auf der verbalen Oberfläche niederschlägt. Mithilfe dieser Partikel als intrapersonellem Koordinierungsmarker kann die Einzelaktivität pünktlich zur nächsten TCU abgeschlossen werden, für die daraufhin beide Hände mobilisiert werden können. Zum anderen zeigt sich die funktionale Differenzierung der händischen Beteiligungsweisen an den Aktivitäten. Ähnlich wie beim Spielen eines Saitenoder Streichinstruments spiegeln beide Hände einander nicht, sondern erfüllen unterschiedlich komplexe Bewegungs- und Handlungsanforderungen mit jeweils eigenen temporalen Logiken: die linke Hand greift und zieht vertikal, während die rechte Hand in Übereinstimmung mit der Sprachproduktion horizontal geschüttelt wird. Mit dem Übergang von einer multiaktiven zu einer monoaktiven Konfiguration tritt diese Differenzierung in den Hintergrund: beide Hände bearbeiten nun nicht mehr unterschiedliche Aufgaben, sondern ermöglichen gespiegelt und zum Rhythmus des Gesangs synchron die tanzende Beteiligungsweise an der Ensembleaktivität Vorschlag (Tab. 19). ${ }^{47}$

Die hohe intrapersonelle Anforderung bei der simultanen Koordinierung multipler Aktivitäten lässt sich besonders dann gut erkennen, wenn Interagierende keine interpersonelle Koordinierung vornehmen müssen. Dies ist dann der Fall,

46 Die Reaktion des Regisseurs, der den Vorschlag dadurch ablehnt, dass er einen GeGENVoRSCHLAG produziert, wurde bereits in Transkript 6 besprochen. Dadurch, dass er seinen GEGENVoRSCHLAG sowohl inhaltlich als auch koordinativ auf den VoRSCHLAG der Schauspielerin abstimmen kann, zeigt er an, dass die Konzeptvermittlung der Schauspielerin kommunikativ erfolgreich war. 47 In neuropsychologischen Untersuchungen zu bimanueller Koordination beim Menschen (zum Beispiel Kelso 1984, Hazeltine, Weinstein \& Ivry 2008 oder Swinnen \& Gooijers 2015) zeigt sich, dass Menschen dazu neigen, unterschiedliche Handbewegungen zu synchronisieren. Die Partikel „so“ (Z.003) dient aus kognitiver Perspektive vermutlich hier (auch) dazu, eine symmetrische Synchronisation der beiden Hände zu ermöglichen. Dieser spezifische Aspekt der simultanen Koordination wird in Kapitel 6 eingehend betrachtet. 
wenn zwei Einzelaktivitäten gleichzeitig bearbeitet werden, wie im folgenden Transkriptausschnitt. Darin organisiert der Schauspieler seine Einzelaktivitäten IM NEWSFEED LESEN und TRINKEN dergestalt, dass beide Aktivitäten gleichzeitig als TRINKENDES-LESEN möglich werden. Neben dem Schauspieler sind ebenfalls der Hospitant und der Videograph anwesend, die ihren eigenen Einzelaktivitäten nachgehen und kein Interaktionsensemble bilden. Der Fall, der als einziger in dieser Fallkollektion nicht interaktional organisiert ist, zeigt die Praktiken, mit denen Interagierende auch strukturell inkompatible Beteiligungsweisen simultan koordinieren können. Dieses Phänomen wird in Kapitel 7 - dann aber für interaktionale Kontexte - ausführlich betrachtet.

Tab. 20: Blick und rechte Hand als multiple Beteiligungsmarker an der Ensembleaktivität bei simultaner Involvierung in die Einzelaktivität durch SCM.

\begin{tabular}{|c|c|c|c|c|c|c|}
\hline Transkript 16 & a) & b) & c) & d) & e) & f) \\
\hline Einzelakti- & Kernphase & pausiert & Kernphase & pausiert & Kernphase & Kernphase \\
\hline vität: & 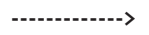 & 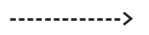 & & ------------.> & ------------.> & ------------.> \\
\hline IM NeWSfEed & vis. \& hap. & Fort. & vis. & Fort.proj. & vis. & vis. \& hap. \\
\hline LESEN & & $\begin{array}{l}\text { proj. via } \\
\text { Körper- } \\
\text { haltung }\end{array}$ & & $\begin{array}{l}\text { via Kör- } \\
\text { per-hal- } \\
\text { tung }\end{array}$ & & \\
\hline \multirow{4}{*}{$\begin{array}{l}\text { Einzelaktivi- } \\
\text { tät: TRINKEN }\end{array}$} & & Startphase & Startphase & Kernphase & Endphase & \\
\hline & & -..-..-.-.>> & -..-..--.-.> & --.--.--.--.> & - 0 & \\
\hline & & vis. \& hap. & hap. & hap. & hap. & \\
\hline & & Beteil. & Beteil. & Beteil. & Beteil. & \\
\hline
\end{tabular}

Analog zum koordinativen Handeln der Schauspielerin im vorherigen Transkript 15 realisiert der Interagierende im folgenden Transkript 16 zwei ko-relevante Aktivitäten, indem er seine Beteiligungsweisen auf die zwei für ihn relevanten Aktivitäten aufteilt. Anders als bei der Schauspielerin verwendet er nur seine rechte Hand, die sich dadurch entweder scrollend an der LESE- oder greifend an der TRINK-Aktivität beteiligen kann. Damit kann er nicht, wie die Schauspielerin, sich mit je einer Hand an einer unterschiedlichen Aktivität beteiligen. Seine simultane Bearbeitung der gleichzeitig relevanten Aktivitäten gelingt in der untenstehenden Sequenz dennoch, da seine Hand ihm eine sensorische Referenz (Mondada 2019a) bzgl. der Position der Tasse ermöglicht, sodass er seine visuelle Modalität auf die LESE-Aktivität ausrichten kann, ohne die Position der Tasse visuell monitoren zu müssen. Der Schauspieler verwendet also sowohl für die Einzelaktivität AM SMARTPHONE LESEN als auch für die Einzelaktivität AUS EINER TASSE TRINKEN haptische Beteiligungsweisen. Zusätzlich blockiert die TRINK-Aktivität in ihrer Kernphase die visuelle Beteiligungsweise an der LESE-Aktivität. Dass der 
Schauspieler beide Aktivitäten trotz multimodaler Inkompatibilität der Beteiligungsweisen simultan koordinieren kann, ist eng damit verbunden, wie er seine Körperhaltung zur Fortsetzungsprojektion einsetzt und wann er welche der Aktivitäten im Rahmen von Mikropausen kurzzeitig zurückstellt. Das Beispiel illustriert damit die Praktiken, mit denen Menschen auch situativ strukturell inkompatible Aktivitäten (LESEN und TRINKEN) simultan koordinieren (vgl. Kap. 7).

\section{Transkript 16: Simultane Beteiligung an zwei Einzelaktivitäten} (Trin Ken und Im Newsfeed LeSEN)

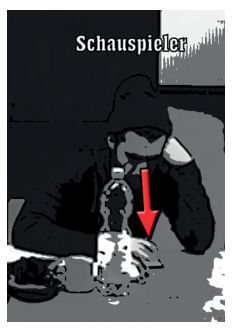

Abb. 16.1: \# ${ }^{1}$.

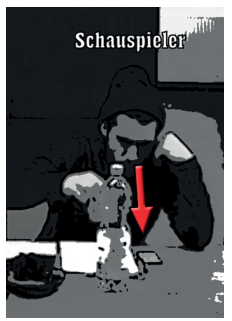

Abb. 16.5: $\#^{5}$.

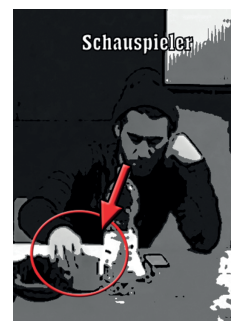

Abb. 16.2: $\#^{2}$.

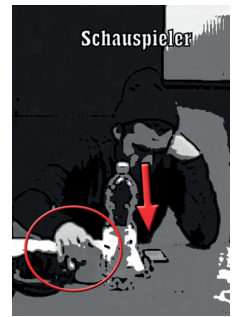

Abb. 16.6: $\#^{6}$.

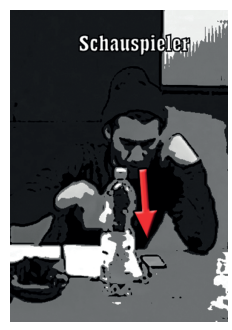

Abb. 16.3: $\#^{3}$.

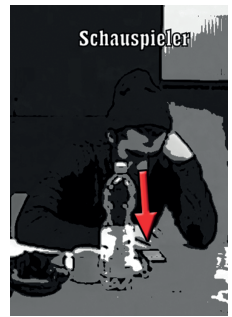

Abb. 16.7: $\#^{7}$.

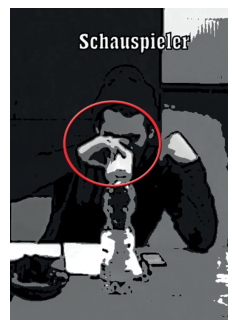

Abb. 16.4: $\#^{4}$.
001
(a) (1.0) $\#^{1}(0.4)$
a+ $(0.4) \#^{2}(0.2)$
aschaut zu Tasse
$a+(0.7) \#^{3}(0.6)$
scm @schaut auf Smartphone
+greift Tasse
@schaut zu Smartphone->
+führt Tasse zu Mund->
002
a+ (1.0) \# $\#^{4}(0.5)$
a+ $(0.2) \#^{5}(0.6) \#^{6}(1.0)+(0.2) \#^{7}(0.8)$
scm @schaut in Tasse
+trinkt aus Tasse
@schaut zu Smartphone----- $--------->>$ +stellt Tasse auf Tisch +

a) Vollzug der LESE-Kernphase via visueller und haptischer Beteiligungsweisen (Abb. 16.1): Der Schauspieler vollzieht IM NEWSFEED LESEN primär dadurch, dass 
er auf die linke Hand gestützt auf das vor ihm liegende Smartphone schaut und mit dem Mittelfinger der rechten Hand durch den Newsfeed scrollt (Abb. 16.1).

b) Pausieren der LESE-Aktivität durch Fortsetzungsprojektion via Körperhaltung bei gleichzeitigem Vollzug der TRINK-Startphase via visueller und haptischer Beteiligungsweisen (Abb. 16.2): Diese Konfiguration löst der Schauspieler auf, indem er seinen Blick vom Smartphone auf die rechts vor ihm stehende Tasse orientiert, während er gleichzeitig seine rechte Hand vom Smartphone hebt und zur Tasse führt. Damit besteht keine aktive Bearbeitung der LESE-Aktivität, während er die TRINK-Aktivität initiiert. Einzig mit seiner unveränderten Körperhaltung zeigt er im Rahmen einer Fortsetzungsprojektion an, dass die Relevanz der LESE-Aktivität weiterhin besteht und die zeitnahe Rückkehr in die working position dieser Aktivität erwartbar ist (Abb. 16.2).

c) Vollzug der LESE-Kernphase via visueller Beteiligungsweise bei gleichzeitigem Vollzug der TRINK-Startphase via haptischer Beteiligungsweise (Abb. 16.3): Die Erwartbarkeit der Fortsetzung der LESE-Aktivität löst er bereits nach 0.6Sekunden ein, als er seinen Blick zurück auf das Smartphone richtet, noch während er die Tasse zum Mund führt (Abb. 16.3). Damit teilt der Schauspieler seine multimodalen Ressourcen so auf die gleichzeitig relevanten Aktivitäten auf, dass er beide Aktivitäten simultan als LESENDE-TRINK-Multiaktivität bearbeiten kann.

d) Pausieren der LESE-Aktivität durch Fortsetzungsprojektion via Körperhaltung bei gleichzeitigem Vollzug der TRINK-Startphase via haptischer Beteiligungsweise (Abb. 16.4): Unterbrochen wird diese gleichzeitige Bearbeitung der LESE-Aktivität durch die physische Beschränkung der Tasse, die es verhindert, dass er während der Kernphase des Trinkens auf die visuelle Beteiligungsweise zurückgreifen und LESE-Aktivität fortsetzen kann (Abb. 16.4).

e) Vollzug der LESE-Kernphase via visueller Beteiligungsweise bei gleichzeitigem Vollzug der TRINK-Endphase via haptischer Beteiligungsweise (Abb. 16.5-6): Sobald die TRINk-Kernphase abgeschlossen ist und die Tasse in der TRINKEndphase wieder zurück auf den Tisch gestellt wird, orientiert sich der Schauspieler erneut auf sein Smartphone und nimmt seine LESE-Aktivität wieder auf (Abb. 16.5). Im Unterschied zum Greifen der Tasse in der Startphase des TRINKENS (Abb. 16.2) vollzieht der Schauspieler das finale Abstellen der Tasse ohne visuelle Beteiligung an dieser Aktivität (Abb. 16.6).

f) Vollzug der LESE-Kernphase via visueller und haptischer Beteiligungsweisen (Abb. 16.7): Der Schauspieler führt daraufhin seine rechte Hand zum Smartphone zurück und nimmt dadurch seine LESE-Gestalt wieder auf (Abb. 16.7). 
Die simultane Koordinierung der beiden Einzelaktivitäten wird in diesem Fallbeispiel durch die enge Verzahnung der Beteiligungsweisen ermöglicht, die durch eine Aufteilung der multimodalen Ressourcen erreicht wird. Mithilfe des Blicks kann der Schauspieler die LESE- und durch die haptische Beteiligung der Hand die TRINk-Aktivität vollziehen. Dass diese Aufteilung eine Praktik zur Bearbeitung der gleichzeitigen Relevanz der beiden Aktivität ist, wird dadurch ersichtlich, dass der Schauspieler zu seinem multimodalen Status Quo zurückkehrt, sobald die TRINK-Aktivität abgeschlossen ist. Im Zuge dessen pausiert der Schauspieler die LESE-Aktivität, als die Beteiligungsweisen der ko-relevanten multiplen Aktivitäten um dieselben multimodalen Ressourcen konkurrieren und sich dadurch strukturell inkompatibel zu einander verhalten. Dieser Fall tritt in dem Moment ein, in dem er die Position der Tasse in der Startphase des TRINKENS zunächst identifizieren muss, bevor er sie zum Mund führen kann. Darüber hinaus zeigen beide Aktivitäten neben der engen multimodalen Verzahnung eine stark wechselwirkende Beziehung. Dies zeigt sich zum einen im Alternieren zwischen Bearbeitung und Pausierung der LESE-Aktivität, die davon abhängig ist, ob die visuelle Ressource Blick verfügbar bzw. im Gebrauch der Konkurrenzaktivität ist. So ist es für den Schauspieler strukturell unmöglich, nach unten auf das Smartphone zu schauen, während er seine Tasse in der Kernphase des TRINkENS anhebt. Auf der einen Seite weist das engagement display an der TRINK-Aktivität in ihrem Verlauf eine sukzessive Herabstufung der multimodalen Beteiligungsmarker auf. Sind in der Startphase der TRINK-Aktivität noch visuelle und haptische Beteiligungsweisen zur Bearbeitung notwendig, können Kern- und Endphase ohne visuelle Beteiligung bearbeitet und abgeschlossen werden. Dies ermöglicht es dem Schauspieler wieder in die LESE-Aktivität einzusteigen, noch bevor die TRINK-Aktivität beendet ist. Die simultane Koordination multipler Einzelaktivitäten geht also mit einer engen Verzahnung der Aktivitäten einher, die eine Aufteilung der multimodalen Ressourcen auf die jeweiligen Aktivitäten zur Folge haben können. Der daraus resultierenden Verfügbarkeitsanforderung der Ressourcen für die Bearbeitung der Aktivitäten begegnet der Interagierende im Fallbeispiel dadurch, dass er Aktivitäten mit nichtverfügbaren Ressourcen für den Zeitraum der Nichtverfügbarkeit pausiert. Mithilfe dieser intrapersonellen Organisation der Beteiligungsweisen vermag der Schauspieler beide gleichzeitig relevanten Einzelaktivitäten simultan zu bearbeiten. Auf diese Weise bleiben beide Aktivitäten im Vordergrund, während die Bearbeitung mit der strukturellen Kompatibilität in der jeweiligen Aktivitätsphasen einhergeht.

Deutlich komplexer wird die simultane Koordinierung, wenn Teilnehmende darüber hinaus interpersonelle Beteiligungsweisen an multiplen Ensembleaktivitäten organisieren müssen. Bislang wurden ausschließlich Ensembleaktivitäten mit verbaler Beteiligungsweise betrachtet. Dass Teilnehmende auch sprachfreie 
Aktivitäten als Ensembleaktivitäten organisieren und diese mit anderen Ensembleaktivitäten koordinativ kombinieren, zeigt das folgende Fallbeispiel. In diesem ERKLÄRT die Regieassistentin der Schauspielerin die aktuelle Stückfassung ${ }^{48}$, die sie ihr gleichzeitig AUSHÄNDIGT (vgl. für Objektübergaben als sozial organisierte Aktivitäten Due \& Trærup 2018; Tuncer \& Haddington 2019). Beide Interagierende organisieren die Ensembleaktivitäten als eine interpersonell aufeinander abgestimmte, emergente Multiaktivität des ERKLÄRENDEN-AUSHÄNDIGENS. Die AUSHÄNDIGUNG startet in der untenstehenden Sequenz, nachdem die Regieassistentin (ASS) und die Schauspielerin (SCW) gemeinsam die Probebühne betreten. Zu diesem Zeitpunkt sind bereits mehrere Theaterschaffende anwesend, die in verschiedene Aktivitäten involviert sind. Für das folgende Fallbeispiel ist die parallel stattfindende Ensembleaktivität zwischen Schauspieler (SCM) und Dramaturg (DRA) relevant. Sie bearbeiten im Rahmen einer BERATUNGS-Ensembleaktivität ein Problem des Schauspielers, der sich beim Dramaturgen über zu spät angewiesenes Gehalt und hohe bürokratische Hürden beklagt. Die Bearbeitung dieser Ensembleaktivität als schisming wird in der Organisation der AusHÄNDIGUNGsEnsembleaktivität sichtbar und hat interaktionale Folgen für die simultane Koordinierung der Ensembleaktivitäten von Schauspielerin und Regieassistenz.

Im Laufe der Situation beteiligt sich die Schauspielerin an drei Ensembleaktivitäten, die sie jeweils so koordiniert, dass sie zeitgleich stets in solche Aktivitäten involviert ist, die jeweils strukturell kompatibel zueinander sind. Sowohl für die Partizipation an der BEGRÜßUNG als auch die ERKLÄR-Aktivität greift die Schauspielerin auf verbale und visuelle Beteiligungsweisen zurück, sodass es ihr möglich ist, beide Aktivitäten jeweils mit der Aktivität STÜCKFASSUNG AUSHÄNDIGEN simultan zu koordinieren, für die sie lediglich gestische bzw. haptische Beteiligungsweisen verwendet (Tab. 21). Die simultane Koordination solcher strukturell kompatiblen Ensembleaktivitäten wird in Kapitel 6 ausführlich diskutiert.

\footnotetext{
48 Die aktuelle Stückfassung ergibt sich aus den Improvisationen, Diskussionen und dem szenischen Probieren. Grundlage dafür sind die Mitschriften der Regieassistenz, die diese nach Probenschluss abtippt und an den Regisseur weiterleitet. Dieser erstellt daraus eine neue Textfassung, die er oft noch in derselben Nacht an die Schauspielerinnen weiterleitet. Insbesondere für die Schauspieler*innen resultiert daraus ein konstantes Textlernen vor, während und nach der Probe. Die letzte Stückfassung haben Schauspieler und Schauspielerin in der 2. Hauptprobe (Probe vor der Generalprobe) erhalten.
} 
Tab. 21: Geste und Sprache-Körperorientierungsgestalt als multiple Beteiligungsmarker an zwei Ensembleaktivitäten durch SCW.

\begin{tabular}{|c|c|c|c|}
\hline Transkript 17 & a) & b) & c) \\
\hline Ensembleaktivität: & engagement display & engagement display & engagement display \\
\hline STÜCKFASSUNG & 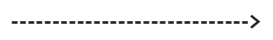 & 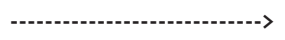 & 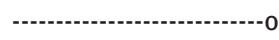 \\
\hline \multirow[t]{2}{*}{ AUSHÄNDIGEN } & gestische & gestische & haptische \\
\hline & Beteiligungsweise & Beteiligungsweise & Beteiligungsweise \\
\hline Ensembleaktivität: & & engagement display & \\
\hline \multirow[t]{3}{*}{ BEGRÜßEN } & & 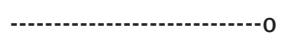 & \\
\hline & & verbale \& visuelle & \\
\hline & & Beteiligungsweisen & \\
\hline Ensembleaktivität: & & & engagement display \\
\hline \multirow[t]{3}{*}{ ERKLÄREN } & & & 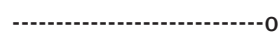 \\
\hline & & & verbale \& visuelle \\
\hline & & & Beteiligungsweisen \\
\hline
\end{tabular}

\section{Transkript 17: Simultane Beteiligung an zwei Ensembleaktivitäten (ERKLÄREN Und STÜCKFASSUNG AUSHÄNDIGEN)}

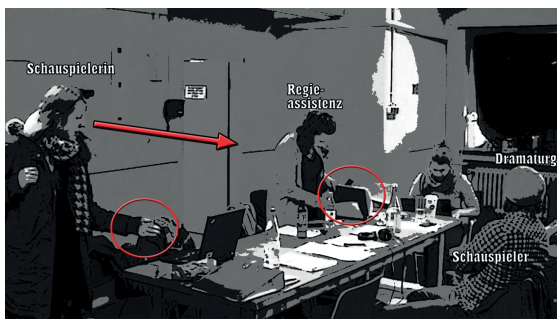

Abb. 17.1: SCW hält Hand auf/schaut zu SCM \& DRA.

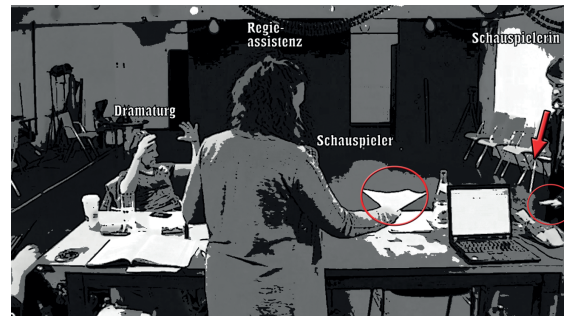

Abb. 17.2: ASS hält SCW die Blätter hin.

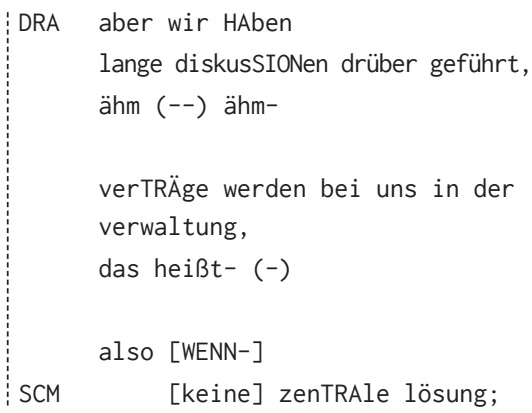




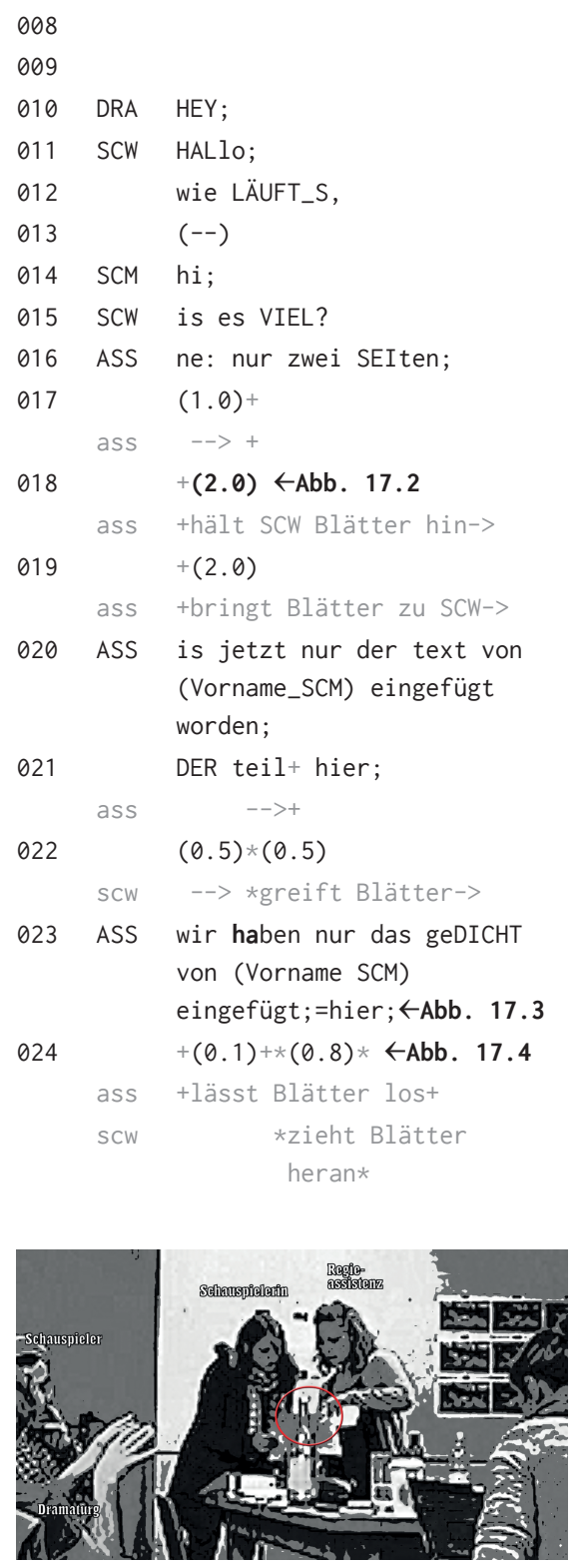

Abb. 17.3: ASS zeigt auf die neue Textstelle.

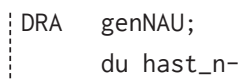

je nachdem was man gerade BRAUCHT,

verhandel ich mit DEN die verträge,

je nach funkTION (-) äh geht_s im besten falle hier

von einem zimmer ins nächste;

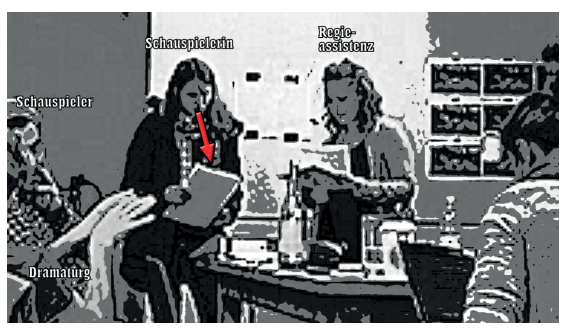

Abb. 17.4: SCW zieht die Stückfassung zu sich heran. 
a) engagement display bzgl. der Aktivität AUSHÄNDIGEN via gestischer Beteiligungsweise (Z.001-009): Zu Beginn des Transkriptausschnitts liefert die Schauspielerin einen Account für ihr spätes Kommen (Z.001-2), während sie sich am Tisch entlang Richtung ihres Platzes bewegt. Die Entschuldigung wird von der Regieassistentin angenommen („ALles gut;“, Z.003), die zeitgleich damit beginnt, in ihren Unterlagen zu suchen. Dass es vorher eine Absprache zwischen Schauspielerin und Regieassistentin hinsichtlich der aktuellen Stückfassung gegeben haben muss, wird deutlich, als die Schauspielerin ihre Hand mit der Handfläche nach oben in Richtung der Regieassistentin hält (Z.005) und dadurch eine Übergabe der Textseiten per gestischer Beteiligungsweise einfordert. Damit beteiligen sich Schauspielerin und Regieassistentin verbal abstinent an der AushÄNDIGUNGs-Ensembleaktivität: Die Regieassistentin bearbeitet suchend eine Anforderung der Ensembleaktivität (um die Textseiten AUSHÄNDIGEN zu können, muss sie diese zunächst finden) und die Schauspielerin hält weiterhin ihre „empfangende“ Geste in Richtung der Regieassistentin aufrecht (Abb. 17.1).

b) engagement display bzgl. der Aktivität AUSHÄNDIGEN via gestischer Beteiligungsweise bei gleichzeitigem engagement display bzgl. der Aktivität BEGRÜ$\beta E N$ via visueller und verbaler Beteiligungsweisen (Z.010-014): In der multimodalen Gestalt der „empfangenden“ Geste beginnt die Schauspielerin die Ensembleaktivität zwischen dem Dramaturgen und dem Schauspieler zu monitoren. Ihre dadurch entstehende simultane Beteiligung an zwei Aktivitäten organisiert sie mit derselben Praktik, die bereits in den Trankskripten 15 und 16 zum Einsatz kam: die Aufteilung der benötigten Beteiligungsweisen auf unterschiedliche Aktivitäten je nach struktureller Kompatibilität. Mithilfe der aufrechterhaltenen Geste zeigt sie ihre fortwährende Forderung nach den Textseiten der AushäNDIGUNGs-Ensembleaktivität an, während sie mit visueller Beteiligungsweise ihr engagement display an der BERATUNGS-Ensembleaktivität zwischen dem Dramaturg und dem Schauspieler herstellt. Es entsteht die Gestalt einer AUSHÄNDIGENDEN-BEGRÜßungs-Multiaktivität. Dieses Beteiligungsdisplay wird vom Dramaturgen bemerkt und zieht eine Begrüßungssequenz nach sich (Z.010-14), die die BERATUNG unterbricht und kurzzeitig eine triadische BEGRÜßUNGS-Ensembleaktivität mit der nun auch verbal beteiligten Schauspielerin formt.

c) engagement display bzgl. der Aktivität AUSHÄNDIGEN via haptischer Beteiligungsweise bei gleichzeitigem engagement display bzgl. der Aktivität ERKLÄREN via visueller und verbaler Beteiligungsweisen (Z.015-024): Nachdem die drei Beteiligten Grüße ausgetauscht haben, zerfällt diese Ensembleaktivität zeitgleich wieder 
in die zwei parallele Ensembleaktivitäten BERATEN und AUSHÄNDIGEN DER AKTUELLEN STÜCKFASSUNG. Dazu recycelt der Dramaturg sein sprachliches Material (,du hast“ Z.009/13/15), während sich die Schauspielerin an die Regieassistentin mit einer Frage nach dem Umfang der AUSZUHÄNDIGENDEN Unterlagen wendet (Z.015) und damit eine ERKLÄRUNG als weitere Ensembleaktivität mit der Regieassistentin etabliert. Diese beantwortet die Frage („ne: nur zwei Seiten“, Z.016) ohne die Schauspielerin anzuschauen, findet die angesprochenen Seiten (Z.017) und hält sie der Schauspielerin entgegen (Abb. 17.2). Die Regieassistentin bietet der Schauspielerin damit die Übergabe der Textseiten an. Die Schauspielerin hält zu diesem Zeitpunkt zwar immer noch ihre „empfangende“ Geste und damit ihre Forderung aufrecht, monitort aber wieder die Ensembleaktivität von Dramaturg und Schauspieler und kann damit das sprachfreie Angebot der Textseiten nicht wahrnehmen. Die Regieassistentin löst dieses praktische Problem, indem sie die Distanz zwischen sich und der Schauspielerin reduziert. Sie verbindet diese proxemische Beteiligungsweise an der AusHäNDIGUNGs-Ensembleaktivität mit einem verbalen Beitrag einer ERKLÄRUNG (Z.019-020), die thematisch an die vorherige Frage der Schauspielerin nach dem Umfang anknüpft. Dazu weist die Regieassistentin zunächst global auf die relevante Modifikation der Stückfassung hin (,is jetzt nur der text von von (Vorname SCM) eingefügt worden“, Z.020), bevor sie lokal auf die betreffende Textstelle auf der Papierseite verweist („DER teil hier“, Z.021). Damit ist zwar die Arbeitsdistanz für die AusHÄNDIGUNG hergestellt, die Übergabe aber noch nicht vollzogen. Die Schauspielerin reagiert darauf, indem sie die ihr gereichten Textseiten greift (Z.022). Es kommt allerdings noch zu keiner Übergabe, da die Regieassistentin die Blätter weiterhin festhält. Stattdessen reformuliert die Regieassistentin ihre ERKLÄRUNG (Z.023). Sie reagiert dabei auf die veränderte Verfügbarkeit der Beteiligungsweisen, die sich daraus ergibt, dass nun Schauspielerin und Regieassistentin die Stückfassung mit jeweils einer Hand festhalten. Dies ermöglicht es der Regieassistentin nun neben verbalen Referenzpraktiken („das geDICHT“ / „hier“, Z.023) auch gestisches Zeigen (Abb. 17.3) einzusetzen. Nachdem die ERKLÄRUNG der Änderungen abgeschlossen ist, lässt die Regieassistentin die Blätter los (Z.024), was es der Schauspielerin ermöglicht, die Stückfassung zu sich zu ziehen (Abb. 17.4) und damit die AusHÄNDIGUNG abzuschließen. Schauspielerin und Regieassistenz koordinieren in dieser Sequenz gemeinsam die zwei gleichzeitig relevanten Ensembleaktivitäten AUSHÄNDIGUNG DER AKTUELLEN STÜCKFASSUNG und ERKLÄREN dergestalt, dass die Multiaktivität AUSHÄNDIGENDE-ERKLÄRUNG entsteht. Dabei greifen beide Teilnehmerinnen einerseits auf die gleiche Praktik der Ressourcenaufteilung zurück, wie sie bereits in den vorherigen Transkripten beobachtet werden konnten: So hält die Schauspielerin mit ihrer aufrechterhaltenen Geste ihre Forderung nach den Textseiten aufrecht, während sie sich verbal und mit ihrer Körperorientierung an den 
Ensembleaktivitäten BEGRÜßEN und kurz darauf am ERKLÄREN beteiligt. Dadurch teilt sie ihre Beteiligungsweisen so auf, dass eine simultane Bearbeitung möglich wird. Die Beteiligungsweisen der beiden Interagierenden unterscheiden sich andererseits sowohl in Hinblick auf die strukturellen Anforderungen der Aktivitäten als auch hinsichtlich der realisierenden Handlungen. Die Schauspielerin ist bei beiden Aktivitäten insofern die Nutznießerin, als ihr sowohl etwas ausgehängt als auch erklärt wird. Diametral dazu ist die Regieassistentin in beiden Aktivitäten diejenige, die etwas aushändigt bzw. erklärt. Reicht daher für die Schauspielerin die aufrechterhaltende Geste aus, um ihre fortbestehende Involvierung in die AushäNDIGUNG anzuzeigen, greift die Regieassistentin auf eine Vielzahl unterschiedlicher Beteiligungsmarker zurück. Diese umfassen Praktiken der Auge-Hand-Koordination bei der Suche der Textseiten in der Startphase der Aktivität, die Positionsveränderung zur Vorbereitung der Übergabe der Textseiten in der Kernphase und schließlich das geordnete Loslassen der durch die Schauspielerin angenommenen Textseiten in der Endphase (Tab. 22). Die enge Verknüpfung der Aktivitäten wird darüber hinaus nicht nur in der Verzahnung der aufgeteilten multimodalen Ressourcen sichtbar, sondern auch in der zeitlichen Bearbeitung. Denn erst mit Abschluss ihrer verbalen Beteiligungsweise an der ERKLÄRUNG lässt die Regieassistentin die Textseiten los und ermöglicht der Schauspielerin den zweiten Paarteil Annahme der Textseiten (Z.024). Indem die Regieassistentin die Textseiten festhält, verzögert sie den Abschluss der AusHäNDIGUnG, bis sie ihre ERKLÄRUNG zu einem möglichen Abschluss gebracht hat. Textseitenreichen, -festhalten und -loslassen sind dabei Praktiken der Regieassistentin, mittels derer sie den Zeitpunkt der Übergabe und Annahme der Textseiten organisiert.

Tab. 22: Situativ variierte multiple Beteiligungsmarker zur simultanen Beteiligung an zwei Ensembleaktivitäten durch ASS.

\begin{tabular}{|c|c|c|c|c|c|}
\hline \multicolumn{6}{|l|}{ Transkript 17} \\
\hline \multirow{3}{*}{$\begin{array}{l}\text { Ensembleakt.: } \\
\text { STÜCKFASSUNG } \\
\text { AUSHÄNDIGEN }\end{array}$} & engag. dis. & engag. dis. & engag. dis. & $\begin{array}{l}\text { Fortset- } \\
\text { zungsproj. }\end{array}$ & engag. dis. \\
\hline & ------------.> & -------------->> & 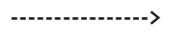 & 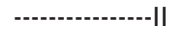 & >------------o \\
\hline & $\begin{array}{l}\text { hap. \& vis. } \\
\text { Beteil. }\end{array}$ & $\begin{array}{l}\text { hap. \& vis. } \\
\text { Beteil. }\end{array}$ & $\begin{array}{l}\text { proxemische } \\
\text { Beteili- } \\
\text { gungsweise }\end{array}$ & $\begin{array}{l}\text { hap. Beteil. } \\
\text { (festhalten) }\end{array}$ & $\begin{array}{l}\text { hap. Beteil. } \\
\text { (loslassen) }\end{array}$ \\
\hline \multirow{5}{*}{$\begin{array}{l}\text { Ensembleakt.: } \\
\text { ERKLÄREN }\end{array}$} & & engag. dis. & engag. dis. & engag. dis. & \\
\hline & & --------------> & 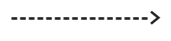 & 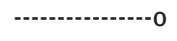 & \\
\hline & & verbale & verbale \& & Verb., vis., & \\
\hline & & Beteil. & visuelle & gest. & \\
\hline & & & $\begin{array}{l}\text { Beteili- } \\
\text { gungsweise }\end{array}$ & $\begin{array}{l}\text { Beteili- } \\
\text { gungsweise }\end{array}$ & \\
\hline
\end{tabular}


Indem sie ERKLÄRT, was sie AUSHÄNDIGT und AUSḦ̈NDIGT, was sie ERKLÄRT, entsteht ein enges Geflecht beider Ensembleaktivitäten als Multiaktivität. Beide Aktivitäten werden hier von den Teilnehmenden nicht als konkurrierende Relevanzen, sondern als unterschiedliche Verfahren zur Bearbeitung der gleichen Interaktionsaufgabe Wissensausgleich bzgl. eines Arbeitsdokuments behandelt. Dies zeigt sich insbesondere in den engagement displays, die durch das Aufteilen der Beteiligungsweisen auf die jeweils ko-relevanten Aktivitäten entstehen.

Wie die Analysen zeigen konnten, macht die Verteilung der Beteiligungsweisen auf gleichzeitig relevante Aktivitäten die simultane Bearbeitung derselben möglich. Um eine solche simultane Koordinierung zu ermöglichen, greifen Teilnehmende auf Verzögerungsstrategien zurück (Transkript 15) oder pausieren Aktivitäten, deren multimodale Ressourcen als Beteiligungsmarker zeitweilig nicht verfügbar sind (Transkript 16). Indem Teilnehmende multiple Beteiligungsmarker einsetzen, kommt es (anders als in den Transkripten 12-14 mit nur einem singulären Beteiligungsmarker) zu keinem Wechsel in der Teilnehmendenrahmung. Stattdessen ermöglicht die simultane Realisierung multipler, gleichzeitig relevanter Aktivitäten schließlich die Bearbeitung einer komplexen Interaktionsaufgabe (Transkript 17).

\subsection{Zwischenfazit: Praktiken der Koordination zur Bearbeitung gleichzeitiger Relevanzen multipler Aktivitäten}

Das interaktionale Phänomen der multiplen Aktivitäten existiert unabhängig von der Verteilung des Rederechts oder anderen verbalen Ordnungsprinzipien. In multiple Aktivitäten können sowohl verbal abstinente wie auch verbal aktive Beteiligte involviert sein. Dabei ist in den besprochenen Fällen dieses Kapitels eine verbale Beteiligung an einer Ensembleaktivität erwartbarer (und auch einklagbarer) als an einer Einzelaktivität - allerdings keine notwendige Voraussetzung. Involvierungen in Ensembleaktivitäten zeigen Interagierende neben verbalen Beiträgen vor allem über aufeinander bezogene Orientierungen (besonders Blickkontakt und Körperausrichtung), Gestikulation und Rezipientensignale an. Situationen mit multiplen Ensembleaktivitäten können innerhalb derselben Teilnehmendenrahmung hergestellt werden (z. B. das ERKLÄRENDE-AUSHÄNDIGEN der aktuellen Stückfassung in Transkript 17) oder mehrere Teilnehmendenrahmungen nach sich ziehen, die jeweils separat voneinander bearbeitet werden (z. B. in Transkript 7 das Ausscheiden des Schauspielers aus dem Interaktionsensemble nach dem verlorenen Wettkampf um das Rederecht oder in Transkript 17 die Beteiligung der Schauspielerin an der Begrüßung mit Schauspieler und Dramaturg, während sie gleichzeitig ihre Forderung nach der Stückfassung an die 
Regieassistentin aufrechterhält). Die Koordination multipler Aktivitäten unterscheidet sich zum einen je nachdem, ob und wie viele der Aktivitäten intersubjektiv ausgerichtet sind und zum anderen, mit welchen Beteiligungsweisen Teilnehmende engagement displays anzeigen. An der Aufrechterhaltung der Aktivitäten sind sowohl diejenigen Interagierenden beteiligt, die mit ihren Beteiligungsweisen die Aktivitäten als principal voranbringen (z. B. durch verbale oder gestische Beteiligung) als auch jene, die lediglich engagement display anzeigen (z. B. durch visuelle Beteiligungsweisen). Denn wie die Analysen gezeigt haben, erfordert die Herstellung und das Aufrechterhalten eines engagement displays bzgl. einer Ensembleaktivität aufgrund der intersubjektiven Ausrichtung eine konstante interpersonelle Koordinierung. Während verbale Beteiligungsmarker ebenfalls das Fortbestehen einer Involvierung anzeigen können, ist es vor allem der Blickkontakt, der von den Beteiligten als Beteiligungsanzeige eingefordert wird. Diese Beobachtung, die sich mit den Befunden der Blickforschung (Argyle \& Cook 1976; Argyle et al. 1980; Goodwin 1980a; Goodwin 1980b; Kendon 1967; Rossano 2013) deckt, kommt insbesondere dann zum Tragen, wenn sich im Rahmen gleichzeitiger Relevanz multipler Aktivitäten der Blickkontakt als visueller Beteiligungsmarker an einer Ensembleaktivität auf ein Fokusobjekt einer Einzelaktivität verschiebt. Im Gegensatz dazu unterscheiden sich die Beteiligungsmarker in Einzelaktivitäten systematisch davon, da sie nur wenig intersubjektiv ausgerichtet sind und zu einem hohen Anteil haptische Praktiken bzw. visuelle Orientierung auf das Fokusobjekt der Einzelaktivität beinhalten. Wenn Teilnehmende nicht in der Lage sind, solche engagement displays für alle gleichzeitig relevanten Aktivitäten gleichermaßen anzuzeigen (simultane Koordination), greifen sie auf die Koordinationspraktiken des/der Abbruchs/Wiederaufnahme (serielle Koordination) und Pausierens/Fortsetzens (quasi-simultane Koordination) zurück. Entscheidend dafür, inwiefern Teilnehmende engagement displays für alle gleichzeitig relevanten Aktivitäten anzeigen können, ist die strukturelle Kompatibilität der Beteiligungsweisen, mit denen Teilnehmende die jeweiligen Aktivitäten vollziehen. Wenn Beteiligungsweisen für die ko-relevanten Aktivitäten gleichermaßen verwendet werden, können Teilnehmende die situativ weniger relevante Aktivität abbrechen oder pausieren. Beim Pausieren wird eine der Beteiligungsweisen der konkurrierenden Aktivität eingefroren. Die betreffende Beteiligungsweise steht dann für keine der ko-relevanten Aktivitäten zum Handlungsvollzug zur Verfügung, zeigt aber via Fortsetzungsprojektion an, dass die pausierte Aktivität im Vordergrund immer noch relevant ist und zeitnah fortgesetzt werden kann, sobald der Grund für die Pausierung überwunden wurde. Wird eine Beteiligungsweise einer Aktivität darüber hinaus als Beteiligungsweise an einer anderen Aktivität notwendig, brechen Interagierende eine der konkurrierenden Aktivitäten ab, wobei die Relevanz der betreffenden Aktivität in den Hintergrund verscho- 
ben wird und erst bei Wiederaufnahmen erneut sichtbar wird. Je nach situativer Anforderung können dabei sowohl Einzel- als auch Ensembleaktivitäten abgebrochen werden. Aktivitäten sind also keine vorgeformten Einheiten. Vielmehr passen Interagierende ihre Aktivitäten an die situierten Anforderungen multipler, gleichzeitiger Relevanzen an, indem sie laufende Aktivitäten zugunsten ebenfalls relevanter Aktivitäten abbrechen, pausieren oder so in ihrer multimodalen Gestalt modifizieren - und sich dies in ihren engagement displays einander anzeigen -, dass eine simultane Bearbeitung möglich wird. Die dadurch entstehenden Koordinationsformen lassen sich auf einem Kontinuum zwischen serieller und simultaner Koordination multipler Aktivitäten beschreiben (Tab. 23).

Tab. 23: Verfahren der Koordination multipler Aktivitäten.

\begin{tabular}{lll}
\hline Bearbeitung gleichzeitiger Relevanzen in multiplen Aktivitäten & \\
\hline Serialität & Quasi-Simultanität & Simultanität \\
\hline Praktiken der seriellen & Praktiken der quasi- & Praktiken der simultanen \\
Koordination & simultanen Koordination & Koordination \\
\hline Die Relevanz einer & Die Relevanz einer & Es erfolgt die simultane \\
Aktivität wird zugunsten & Aktivität wird & Bearbeitung mehrerer \\
der Bearbeitung einer & durch Pausierung & gleichzeitig relevanter \\
anderen beendet. & aufrechterhalten. Es & Aktivitäten (=Multiaktivi- \\
& erfolgt aber keine & täten) \\
& Bearbeitung. & \\
\hline - haptische Bindung mit & - haptische Bindung mit & - Alternieren zwischen der \\
Fokusobjekt lösen & Fokusobjekt beibehalten & visuellen Beteiligung \\
- Auflösen der visuellen & - Gestalt der aktuellen & zwischen Fokusobjekt und \\
Beteiligung & Aktivitätsphase „ein- & Beteiligten \\
- Relevantsetzen der & frieren“ & - Einsatz von \\
Konkurrenzaktivität (via & - Überführen & Rückmeldepartikeln als \\
Account) & der pausierten & Beteiligungsmarker \\
Abbrüche verschieben & Beteiligungsweise in & - Aufteilen der multimodalen \\
die Relevanz einer & temporäre working & Ressourcen auf mehrere \\
Aktivität lokal in den & position & Aktivitäten \\
Hintergrund. Die & - Pausierungen & - Emergenz multipler \\
Beteiligungsweisen & verbaler Beiträge & Aktivitäten zu komplexen \\
der abgebrochenen & durch syntaktische & Multiaktivitäten \\
Aktivitäten werden & \& prosodische & Simultanbearbeitungen \\
für andere Aktivitäten & Fortsetzungsprojektion & erlauben es Teilnehmenden \\
verfügbar. Abbrüche & Pausierungen ermöglichen & Aspekte unterschiedlicher \\
ermöglichen damit ein & durchgängige & Projekte oder mehrere \\
Verfahren zur Priorisie- & engagement displays & Aspekte desselben Pro- \\
rung von ko-relevanten & im Vordergrund durch & jekts zu bearbeiten. \\
Handlungskomplexen. & Fortsetzungsprojektionen. & \\
\hline & & \\
& & \\
\hline
\end{tabular}


Serialität entsteht dann, wenn Teilnehmende die Relevanz einer Aktivität zugunsten der Bearbeitung einer gleichzeitig relevanten Aktivität beenden. Dies erreichen Beteiligte unter Rückgriff auf die Koordinationspraktik des Abbrechens. Aktivitätsabbrüche treten dann auf, wenn multiple Aktivitäten unterschiedliche Aufmerksamkeitsfelder (z. B. die visuelle Orientierung eines ${ }^{\star}$ r Teilnehmenden auf eine ${ }^{\star} n$ Ko-Interagierende`n vs. auf ein nur für ihn ${ }^{\star}$ sie zugängliches semiotisches Feld) und/oder physische Aufwendung (z. B. die händische Bearbeitung von Objekten) erfordern. Wie die Analysen gezeigt haben, ist für Teilnehmende dabei weniger relevant wieviel kognitive und körperliche Anstrengung zum Aufrechterhalten der jeweiligen Aktivität notwendig ist, sondern vor allem, inwiefern die notwenigen Beteiligungsweisen innerhalb des interaktionalen Kontextes strukturell integrierbar und miteinander kombinierbar sind. So ist es dem Schauspieler durchaus möglich, die Einzelaktivitäten TRINKEN und AUF DEM SMARTPHONE IM NEWSFEED LESEN miteinander simultan zu koordinieren (Transkript 16), während derselbe Interaktant seine haptische Beteiligungsweise und damit seine SMARTPHONE-Einzelaktivität zugunsten einer Ensembleaktivität BEGRÜßUNG abbricht (Transkript 1), als diese Aktivität eine haptische Beteiligungsweise (in Form eines Handschlags) erfordert. Durch Aktivitätsabbrüche und -wiederaufnahmen entsteht eine Aktivitätsserie, die dadurch gekennzeichnet ist, dass Teilnehmende immer nur ein engagement display bzgl. einer der gleichzeitig relevanten Aktivitäten anzeigen und die jeweils andere Aktivität für die Dauer der Bearbeitung dergestalt zurückstellen, dass keine fortbestehende Involvierung in diese beendete Aktivität beobachtbar ist. Abbrüche von Einzelaktivitäten werden hergestellt, indem haptische und visuelle Bindungen mit relevanten Fokusobjekten aufgelöst werden. Im Unterschied dazu erfolgen Abbrüche von Ensembleaktivitäten zugunsten von Einzelaktivitäten in den in diesem Kapitel besprochenen Fällen dadurch, dass die Interagierenden die verbale und visuelle Orientierung mit den Ko-Interagierenden der Ensembleaktivität aufheben, Nichtverfügbarkeit für die Ensembleaktivität anzeigen oder das engagement display einer Einzelaktivität relevant setzen. Einzel- und Ensembleaktivitäten unterscheiden sich dahingehend systematisch voneinander, dass Einzelaktivitäten ohne Account und ohne intersubjektive Gestaltung abgebrochen bzw. wiederaufgenommen werden können, wohingegen es für die intersubjektiv organisierte Struktur der Ensembleaktivitäten von großer Bedeutung ist, ob die Abbrüche und Wiederaufnahmen von Ensembleaktivitäten accountable gemacht werden.

Zwischen der seriellen und der simultanen Koordinierungsform lässt sich eine Mischform beobachten. In dieser quasi-simultanen Koordinierungsform werden multiple Aktivitäten durch Pausierungen und Fortsetzungen so koordiniert, dass die gleichzeitige Relevanz zweier (oder mehrerer) Aktivitäten im Vor- 
dergrund der Interaktion aufrechterhalten bleibt, jedoch nur eine Aktivität bearbeitet wird. Diese fortbestehende Relevanz einer zeitweise nicht bearbeiteten Aktivität stellen Teilnehmende mithilfe einer Fortsetzungsprojektion her. Diese vollziehen die Interagierenden beim Pausieren von Einzelaktivitäten, indem sie beispielsweise zentrale Handgriffe der Aktivität anhalten, während mindestens ein Element darauf hinweist, dass die Aktivität (jederzeit) wiederaufgenommen werden kann (z. B. indem die Lasche der Tasche mit einer Hand offengehalten wird oder indem der Rucksack nicht abgesetzt wird, während weiterhin die Hände die Riemen umfasst halten). Fortsetzungsprojektionen pausierter Ensembleaktivitäten können in den verbalen Beteiligungsweisen sichtbar werden, indem syntaktisch oder thematisch projiziert wird, dass ein Turn weitergeführt werden wird (Auer 2005; Mondada 2006; Streeck 1995; Stukenbrock 2018b). Haptische oder gestische Beteiligungsweisen an Ensembleaktivitäten können mittels Fortsetzungsprojektion pausiert werden, indem der Abschluss einer Aktivitätsphase verzögert wird. Fortsetzungsprojektionen stellen eine konstante Herstellungsleistungen der Beteiligten dar, mithilfe derer sie pausierte Aktivitäten relevant halten, während sie mit den restlichen nicht angehaltenen Beteiligungsweisen eine konkurrierende Aktivität bearbeiten. Die Auflösung der Fortsetzungsprojektion erfolgt entweder als Fortsetzung der pausierten Aktivität oder mündet in einem Aktivitätsabbruch, wenn die Relevanz der aufrechterhaltenen Aktivität verschwindet oder die eingefrorene Beteiligungsweise für die Bearbeitung der ko-relevanten Aktivität notwendig wird.

Koordinieren Interagierende mehrere gleichzeitig relevante Aktivitäten dergestalt, dass sie diese simultan bearbeiten können, entstehen Formen simultan koordinierter Aktivitäten. Möglich wird dies dann, wenn Teilnehmende ihre multimodalen Ressourcen auf die gleichzeitig relevanten Aktivitäten verteilen. Simultan bearbeitete Aktivitäten stellen neben der steten interpersonellen Koordinierungsleistung vor allem Anforderungen an die intrapersonelle Koordinierung. Diese zeigt primär eine wechselwirkende Verzahnung der multiplen Aktivitäten, wenn Teilnehmende auf Verzögerungspraktiken einzelner Beteiligungsweisen zurückgreifen, um die Simultanität aufrechtzuerhalten. Gleichzeitige Relevanz multipler Aktivitäten muss nicht zwangsläufig mit verschiedenen Zielen bzw. Projekten (z. B. etwas TRINKEN, während Ko-Interagierenden etwas ERKLÄRT wird) einhergehen. Vielmehr ermöglicht den Teilnehmenden eine simultane Realisierung multipler, gleichzeitig relevanter Aktivitäten, komplexe Interaktionsaufgaben zu bearbeiten (z. B. ein Dokument zu ÜBERGABEN, dessen Inhalt man gleichzeitig ERKLÄRT). Die simultan koordinierten, multiplen Aktivitäten emergieren dabei zu Multiaktivitäten, deren Teil-Aktivitäten jeweils einen Aspekt desselben Projekts bearbeiten. Während mit Ausnahme des letzten Tran- 
skripts (Transkript 17) alle diskutierten Fälle gleichzeitige Relevanz unterschiedlicher Projekte beinhaltet haben, werden in den folgenden Kapiteln ausschließlich simultan koordinierte Multiaktivitäten aus strukturell kompatiblen multiplen Ensembleaktivitäten (Kapitel 6) bzw. aus strukturell inkompatiblen multiplen Einzelaktivitäten (Kapitel 7) betrachtet, deren Teil-Aktivitäten unterschiedliche Aspekte derselben Projekte bearbeiten. 